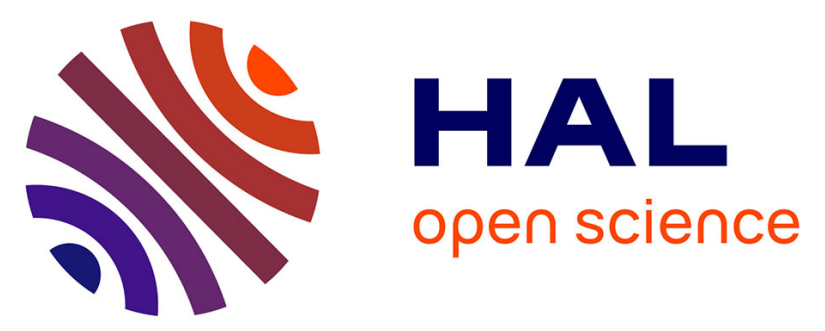

\title{
L'abri sous-roche du Rozel (France, Manche) : un habitat de la phase récente du Paléolithique moyen dans son contexte géomorphologique
}

Brigitte Van vliet-Lanoë, Dominique Cliquet, Patrick Auguste, Elise Folz, David A Keen, Jean-Luc Schwenninger, Norbert Mercier, Philippe Alix, Yves Roupin, Murielle Meurisse, et al.

\section{To cite this version:}

Brigitte Van vliet-Lanoë, Dominique Cliquet, Patrick Auguste, Elise Folz, David A Keen, et al.. L'abri sous-roche du Rozel (France, Manche) : un habitat de la phase récente du Paléolithique moyen dans son contexte géomorphologique. Quaternaire, 2006, 17 (3), pp.207-258. 10.4000/quaternaire.826 . hal-02890035

\section{HAL Id: hal-02890035 https://hal.science/hal-02890035}

Submitted on 19 Oct 2020

HAL is a multi-disciplinary open access archive for the deposit and dissemination of scientific research documents, whether they are published or not. The documents may come from teaching and research institutions in France or abroad, or from public or private research centers.
L'archive ouverte pluridisciplinaire HAL, est destinée au dépôt et à la diffusion de documents scientifiques de niveau recherche, publiés ou non, émanant des établissements d'enseignement et de recherche français ou étrangers, des laboratoires publics ou privés. 


\title{
L'ABRI SOUS-ROCHE DU ROZEL (FRANCE, MANCHE) : UN HABITAT DE LA PHASE RÉCENTE DU PALÉOLITHIQUE MOYEN DANS SON CONTEXTE GÉOMORPHOLOGIQUE
}

\author{
Brigitte VAN VLIET-LANOË ${ }^{1}$, Dominique CLIQUET ${ }^{2}$, Patrick AUGUSTE ${ }^{3}$, \\ Elise FOLZ ${ }^{4}$, David KEEN ${ }^{5}$, Jean-Luc SCHWENNINGER ${ }^{6}$, Norbert MERCIER ${ }^{4}$, \\ Philippe ALIX ${ }^{7}$, Yves ROUPIN ${ }^{8}$, Murielle MEURISSE ${ }^{1} \&$ Hélène SEIGNAC ${ }^{9}$
}

\begin{abstract}
RÉSUMÉ
Situé sur la côte ouest du Cotentin (Manche), le site du Rozel a livré un complexe de deux niveaux d'occupations anthropiques. L'abri sousroche conservait les vestiges d'un habitat comportant des structures de combustion, du mobilier lithique et fait exceptionnel dans le Cotentin des restes osseux. L'ensemble s'inscrit dans une formation dunaire sus-jacente à une plage ancienne.

Initialement attribué à un Périgordien ancien, le site a fait l'objet d'une « relecture » visant à préciser son attribution chronostratigraphique et chronoculturelle.

Le Rozel est actuellement le seul gisement de Basse-Normandie a avoir livré une industrie lithique à composante mixte. Les matières premières utilisées sont le quartz filonien prélevé à l'intérieur même de l'abri, et les galets de silex prélevés dans les cordons littoraux actifs à l'époque. Les générations successives de grands cordons littoraux formés au fur et à mesure de la régression weichsélienne ont été enfouies très rapidement par le massif dunaire, ne permettant plus l'exploitation de leur contenu, riche en nodules propices à la taille. Le débitage apparaît orienté, dans les deux niveaux, vers la production d'éclats prédéterminés (principalement Levallois) et d'enlèvements allongés. Un schéma opératoire laminaire de type paléolithique supérieur a été mis en évidence dans le niveau supérieur, lié à l'occupation principale de l'abri. Le site intègre l'ensemble des gisements à industrie laminaire de gestion volumétrique du début du Dernier Glaciaire.

La faune représentée témoigne de conditions environnementales plutôt tempérées où l'aurochs, les cervidés et dans une moindre mesure les chevaux dominent. L'originalité du site réside dans la présence d'un fragment mandibulaire de morse, associé à l'occupation, témoignant de conditions subarctiques temporaires. L'analyse des vestiges de faune atteste de travaux de boucherie (découpe, fracturation des os longs) et traduit différents modes d'acquisition, ou des traitements particuliers en fonction des espèces.

La convergence des observations effectuées dans les différentes disciplines indique que les occupations du site du Rozel se rapportent à la phase récente du Paléolithique moyen du début du dernier glaciaire weichselien et non au Paléolithique supérieur initial.
\end{abstract}

Mots-clés : Cotentin, dune, plage, habitat, foyers, quartz, silex, Levallois, débitage laminaire volumétrique, environnement tempéré, aurochs, cervidés, morse, Paléolithique moyen

\section{ABSTRACT}

THE ROZEL SHELTER (FRANCE, MANCHE) : A LIVING FLOOR OF THE YOUNGEST MIDDLE PALEOLITHIC IN ITS GEOMORPHOLOGICAL CONTEXT

Located on the Western coast, the Rozel provided an archaeological complex characterized by two occupation levels. The shelter has preserved traces of a habitat with combustion structures, lithic tools, and faunal remains, an exception on the Cotentin peninsula. The settlement is located in littoral dune upon a complex raised beach.

Initially attributed to an Early Perigordian occupation, this site has been reinvestigated from a stratigraphic and cultural point of view. The Rozel shelter is the only occupation in the region presenting a mixed lithic industry. The raw materials used for tools are vein quartz found inside the

\footnotetext{
${ }^{1}$ Laboratoire de Sédimentologie et Géodynamique, FRE 2255 CNRS, Université des Sciences et Technologies de Lille, 59655 Villeneuve-d'Ascq Cedex

${ }^{2}$ Service Régional de l'Archéologie, Direction régionale des Affaires culturelles de Basse-Normandie et PCR «Les Premiers Hommes en Normandie », 13 bis, rue Saint-Ouen, 14052 Caen Cedex04/UMR 6566 CNRS, Université de Rennes 1. E-mail : dominique.cliquet@ culture.gouv.fr ${ }^{3}$ UMR 8018 CNRS, Laboratoire Préhistoire et Quaternaire, Université des Sciences et Technologies de Lille 1, Bâtiment de Géographie, 59655 Villeneuve d'Ascq Cedex. E-mail : Patrick.Auguste@univ-lille1.fr

${ }^{4}$ Laboratoire des Sciences du Climat et de l'Environnement, UMR 1572 CEA-CNRS, Avenue de la Terrasse, Bât. 12, 91 198 Gif-sur-Yvette Cedex

${ }^{5}$ Institute of Archaeology and Antiquity, University of Birmingham, Birmingham B15 2TT, United Kingdom.

${ }_{7}^{6}$ Research Laboratory for Archaelogy, University of Oxford - 6, Keble Road, Oxford OXI 3QJ, United Kingdom.

Institut national de Recherches archéologiques préventives, Rhône-Alpes - 12, rue Maggiorini, 69500 Bron et PCR «Les Premiers Hommes en

Normandie », Direction régionale des Affaires culturelles de Basse-Normandie, 13 bis, rue Saint-Ouen, 14052 Caen Cedex 04.

${ }^{8}$ Le Bourg, 56420 Billio.

${ }^{9}$ Institut national de Recherches archéologiques préventives, Grand-Ouest, Basse-Normandie, Boulevard de l'Europe, 14540 Bourguébus
} 
shelter, and flint cobbles from the active gravel ridges. The successive gravels ridges raised step by step during the weichselian regression were rapidly buried by drift dune sands limiting exploitation of there high content of suitable flint knapping nodules. In the two occupation layers "debitage" seems to be oriented to the production of predeterminated flakes (manly Levallois technique) and elongated flakes. An operating procedure 'laminaire de type paléolithique supérieur', has been revealed in the upper floor, in relation with the main occupation of the site. Le Rozel is an integral part of the series if sites presenting volumetric blade industries from the beginning of the Weichselian.

The preserved fauna attests of mostly temperate environments dominated by wild ox, deer and some horses. The particular interest of this site is the occurrence of a fragment of walrus bone in association with the occupation attesting of temporary subarctic climatic conditions. Analysis of the faunal remains attests to various slaughtering and butchering techniques indicating different methods used according to species. All these multidisciplinary approaches assign all of Le Rozel shelter occupations to the beginning of the Weichselian, not to the Early Upper Palaeolithic.

Key-words : Cotentin peninsula, dune, raised beach, occupation layers, hearths, quartz, flint, Levallois, blade volumetric industry, temperate environment, wild oxen, dears, walrus, middle Palaeolithic

\section{1 - INTRODUCTION}

\section{1 - HISTORIQUE}

La Pointe du Rozel est restée longtemps préservée de l'érosion (fig.1 - carte de Cassini). Ce n'est qu'entre les deux guerres que l'érosion a entamé les dépôts de pentes qui armaient les versants de la pointe.

C'est la découverte entre 1963 et 1967, par Yves Roupin, de plusieurs amas d'ossements en pied de dune sableuse, qui a justifié la conduite d'un sondage (1967) puis une campagne de fouilles (1969).

Ces travaux, effectués sous la direction de Frédéric Scuvée ont fait l'objet, en 1984, de la publication d'une monographie «Paléolithique supérieur en Normandie occidentale : l'abri sous-roche de la pointe du Rozel (Manche) » (Scuvée \& Vérague, 1984).

La révision du site a été motivée par les interrogations formulées à l'occasion de la parution de cette étude. L'analyse géomorphologique du gisement et l'attribution chronoculturelle de l'assemblage lithique ont été contestées.

Depuis, le contexte chronostratigraphique a fait l'objet d'une nouvelle analyse (B. V.V.-L., 1988), le mobilier lithique partiellement observé en 1989 a pu être étudié en 1997 (D.C.), des datations O.S.L. ont été tentées sur la grande dune limitrophe («dune ocre» et «dune humifère ») et dans l'abri (E. F., N. M. et J.-L. $\mathrm{S})$, et enfin, la faune a été ré-examinée (P. A. et D.K.).

La plupart des artefacts lithiques collectés à l'occasion des travaux conduits par F. Scuvée ont pu être analysés (environ $99 \%$ ) ainsi que la quasi totalité de la faune du site.

Le présent article tente de «réviser» ce gisement exceptionnel.

\section{2 - CONTEXTE GÉNÉRAL}

Le site du Rozel est d'un abord difficile, en raison de l'instabilité du massif sableux, plaqué le long de la paléofalaise, qui ne permet pas, à la différence des autres gisements du Cotentin, d'avoir accès à un enregistrement stratigraphique continu (fig. 2 et 3 ). A l'échelle régionale du Cotentin et de la Bretagne, Le Rozel est un des rares sites, avec le Mont Dol (Monnier et al., 1995), Piégu-Pléneuf-Val André, en Baie de
Saint-Brieuc (Monnier, 1985), et La Cotte de Saint Brelade à Jersey (Callow et al., 1986), à conserver de la faune et des mollusques.

Ces gisements sont des sites de pied de falaise ou d'abris, formés par le sapement de la houle dans des falaises de roches relativement cohérentes. L'essentiel de ces falaises daterait de $280 \mathrm{ka}$ (Van Vliet-Lanoë et al., 2000), bien que M. Clet (1983) leur attribue un âge plus récent, avec des évidences de façonnements antérieurs, pouvant remonter au Miocène final (Van VlietLanoë et al., 2002). Pour des facilités de corrélation, nous utiliserons la chronologie isotopique (OIS).

Les abris du Rozel se sont formés à la faveur des nombreuses veines de quartz de filon qui affectent le schiste paléozoïque feuilleté sub-verticalement qui constitue la falaise sud de la pointe du Rozel (fig. 3-b). Ce promontoire d'environ 28 mètres de hauteur est dominé par un plateau qui culmine à 60 mètres. Les trois petites cavités «explorées» par F. Scuvée (TR.67, TR II et TR III) (fig.3-a et b), exposées au sud, étaient masquées par un important massif de sables dunaires (fig. 2). Seul l'abri TR 67 a livré des vestiges d'origine anthropique.

Le site du Rozel se caractérise notamment par une industrie laminaire du Paléolithique moyen, présentant de nombreuses affinités avec certaines séries du gisement de Port Racine (secteurs 1 et 5 ; Cliquet, 1994). Il était donc important de préciser le contexte chronostratigraphique de cet assemblage lithique.

L'analyse microstratigraphique (Van Vliet-Lanoë, 1988) et archéologique (Cliquet, 1994) des sites littoraux du nord Cotentin a permis de repositionner stratigraphiquement les industries du Paléolithique moyen, notamment en fin de OIS 5e (Eemien) et vraisemblablement dans OIS 5c (Brörup - Saint-Germain I).

\section{3 - CONTEXTE STRATIGRAPHIQUE (B. V.V.-L.)}

NB - La stratigraphie présentée sur les figures 4 et 8 a été complétée par rapport à celle proposée en 1988, afin d'intégrer les nouvelles observations (voir § 4.1).

Le bilan sédimentaire reconnu au Rozel revêt un attrait tout particulier en raison de l'exposition plein sud du site, fait exceptionnel pour le Cotentin, de la préservation de faune et de la puissance des formations dunaires fossilisées sous les heads (fig. 4). 

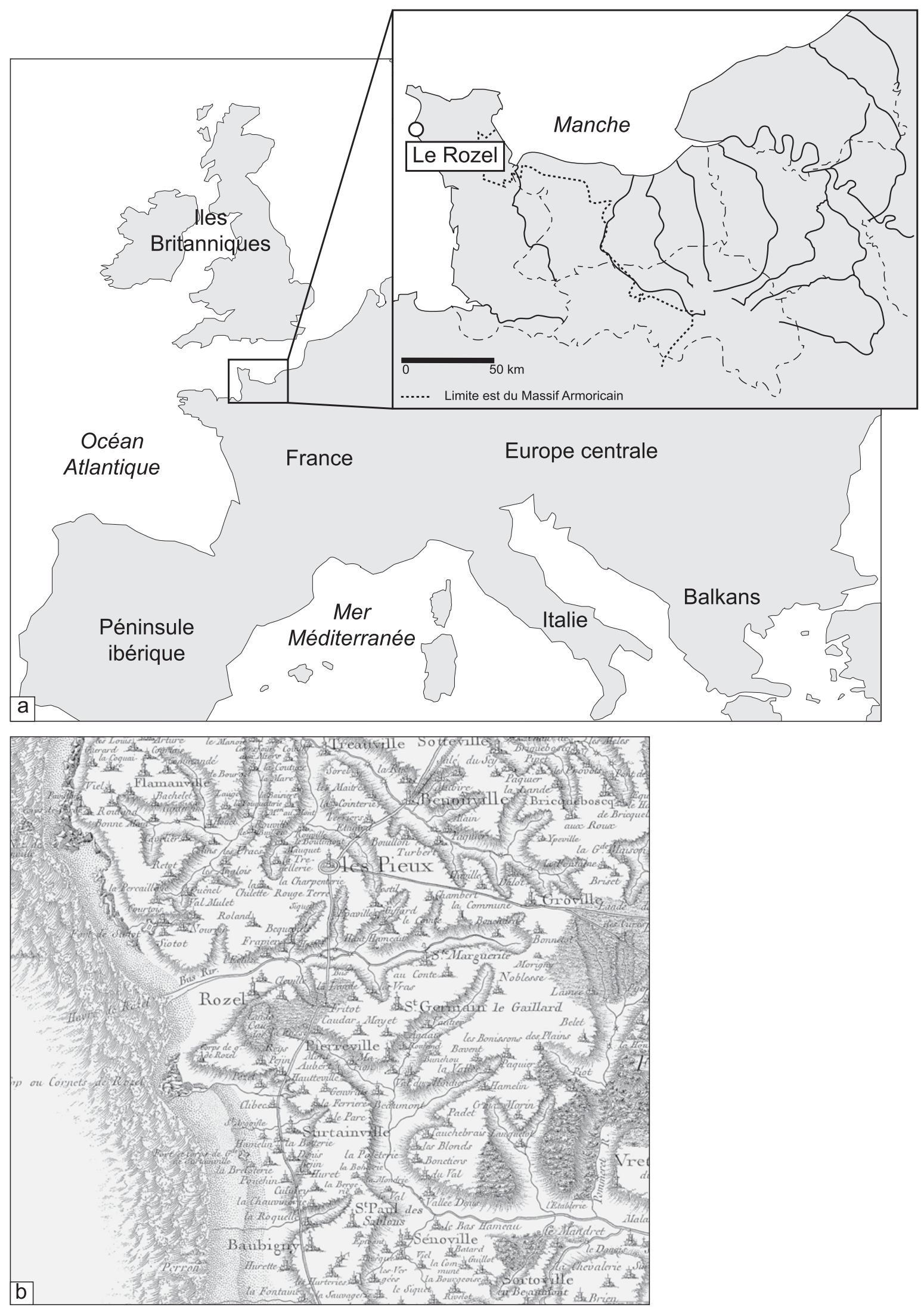

Fig. 1 : Localisation du site archéologique du Rozel et carte de Cassini (XVIII siècle) Fig. 1: Location of Le Rozel archaeological site and Cassini's map (XVIIIth century). 


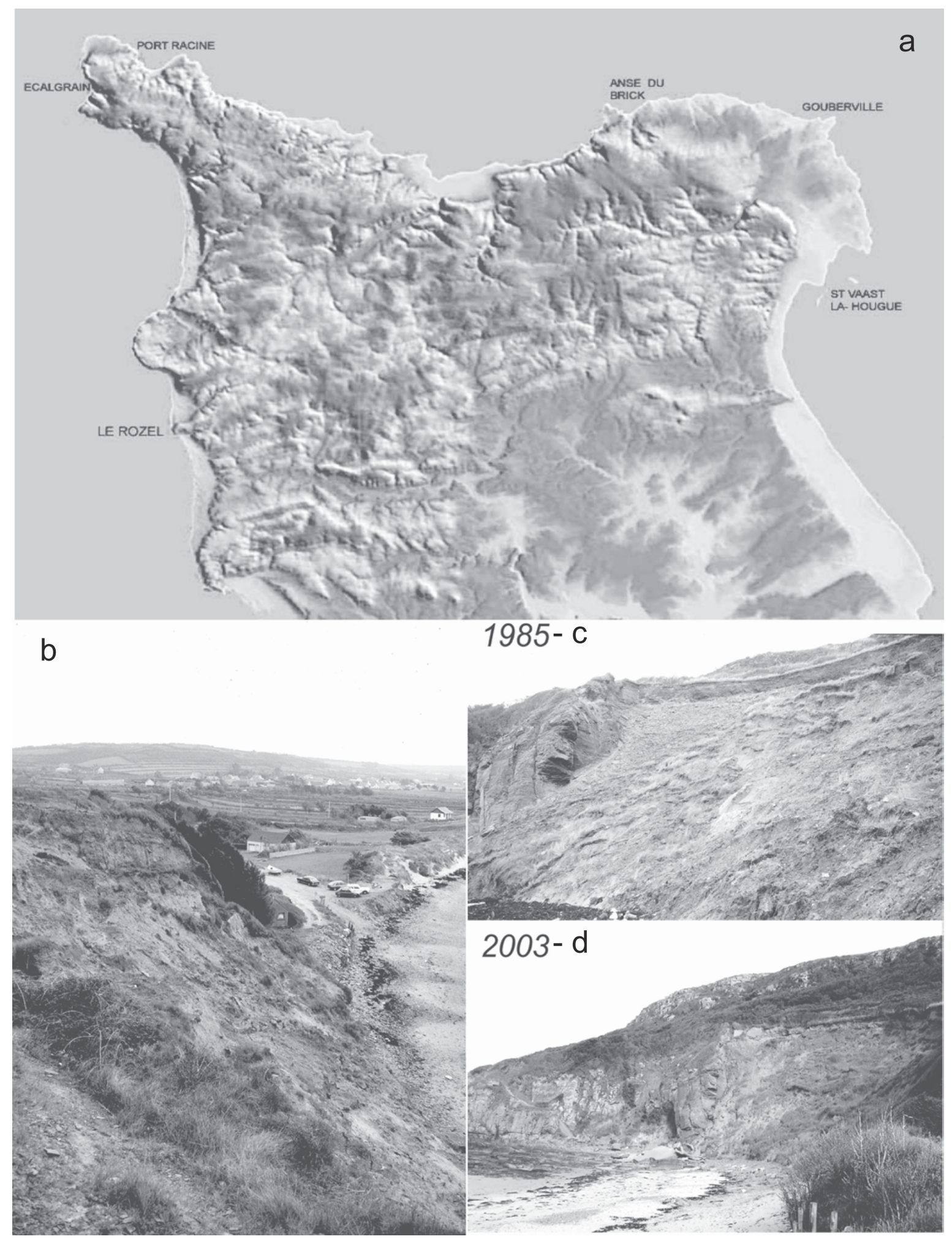

Fig. 2 : (a) Localisation du site dans la morphologie (MNT) et localisation des sites archéologiques contemporains ; (b-d) Evolution de la paléodune entre 1985 et 2003 (clichés B. Van Vliet-Lanoë).

Fig. 2: (a) Location of the Rozel cap in a DEMin relation with other penecontemporaneous archeological sites; (b-d) Evolution of the paleodune between 1985 and 2003. 

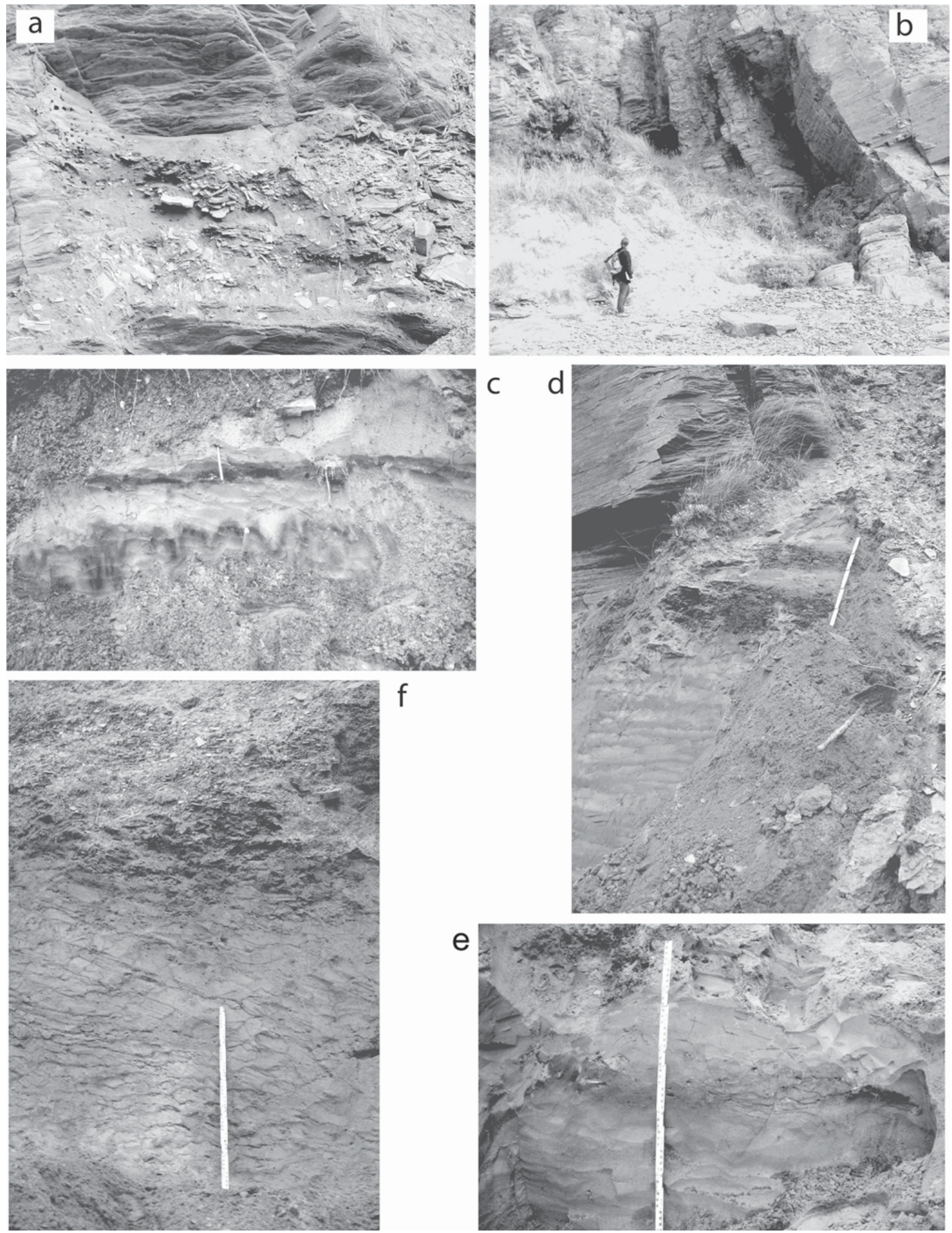

Fig. 3 : (a) Paléosol inférieur sous TR67 ; (b) vue des trois abris (2003) ; (c \& e) podzol in situ soliflué dans D2C ; (d) illuviation en bande dans D1 (paléosol D2b) ; (f) paléosol supérieur (D3d) (clichés B. Van Vliet-Lanoë).

Fig. 3: (a) Lower paleosol below TR67, (b) view of the 3 shelters in 2003; (c \& e) in situ and soliflucted podsol in D2C; (d) banded clay illuviation in DI; (f) Upper paleosol (in the reworked dune) (clichés B. Van Vliet-Lanoë). 


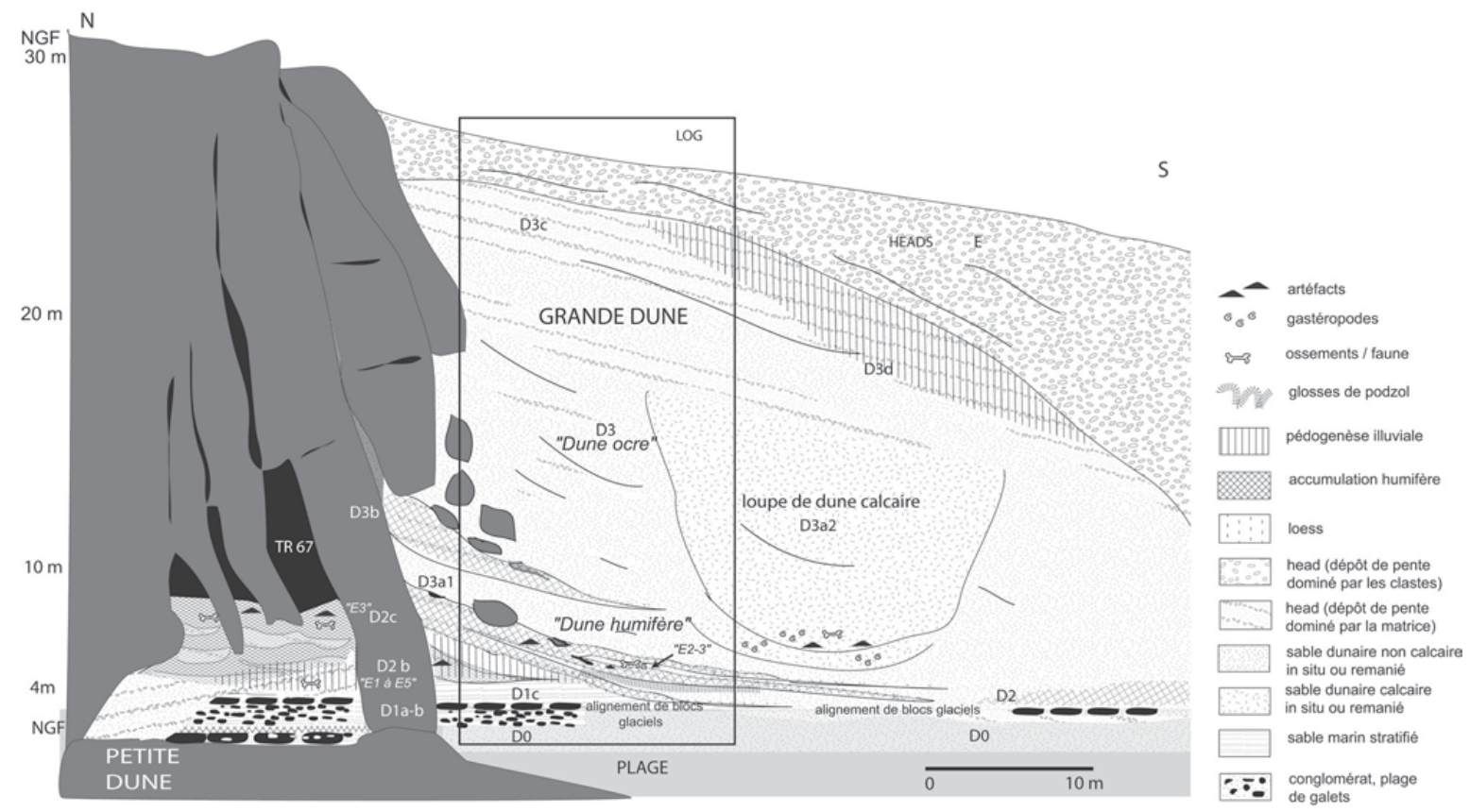

Fig. 4 : Enregistrement sédimentaire composite des abris et de la Grande Dune (d'après Van Vliet-Lanoë, 1988 et les observations récentes). Les unités en « italique » correspondent à celles de Scuvée \& Vérague (1984).

Fig. 4: Composite sedimentary log of the shelters and the Main Dune (from Van Vliet-Lanoë, 1988 and the recent observations). The units in "italic" correspond to those of Scuvée \& Vérague (1984).

Dans la monographie consacrée au gisement, J. Vérague décrit à la base du profil stratigraphique (Scuvée \& Vérague, 1984) (fig. 5) :

1) une plage à gros galets émoussés reposant sur un platier schisteux affleurant à 4 m NGF. Elle est surmontée par un cordon de galets de schistes peu émoussés affecté par un petit sol humifère. Une seconde ligne de blocs plats et émoussés termine cette unité qui a été ensuite enfouie sous des heads schisteux ;

2) une dune humifère surmontée par une dune ocre. A son sommet, un podzol humo-ferrique à glosses (Van Vliet-Lanoë, 1988) se développe, il correspond aux fentes de gel de J. Vérague (in Scuvée \& Vérague, 1984). Son horizon humifère est corrélé par les auteurs au sol d'habitat de l'abri qui est associé à une industrie laminaire attribuée au châtelperronien et d'une faune constituée de bœuf, de cerf et de cheval (détermination M.F.Bonifay). En dehors de l'abri, ce sol est dédoublé par érosion (Scuvée, couches E2 et E3) et remanie du charbon de bois. La malacofaune déterminée par J.-J. Puisségur dénote un milieu assez tempéré, ce qui est compréhensible, compte tenu de l'exposition et du matériel très sableux. Cependant la présence de coquilles est peu conforme avec le développement de la podzolisation.

Sur le terrain, nous avons pu noter que les horizons éluvial (A2) et humifère (A1) du podzol incluent des plaquettes de schistes, ce qui laisse supposer un remaniement de la dune avant la podzolisation.
D'autre part, sur les photos de la publication de F. Scuvée et de J. Vérague, un horizon d'illuviation en bandes ( $\mathrm{Bt}$ ) qualifié de «sable ruisselé » par les auteurs existe sous l'éboulis schisto-limoneux du niveau d'habitat ;

3) une seconde formation dunaire moins complexe et plus épaisse recouvre l'ensemble. D'abord massive (Scuvée, unité F1), elle passe à un second "faciès ruisselé » (Scuvée, unité F2) tronqué, par le head. L'étude malacologique de la base de ce massif dunaire (J.-J. Puisségur), normalement décarbonaté, révèle un contexte un peu plus chaud et plus steppique qu'en E2-3.

Nous avons pu observer (Van Vliet-Lanoë, 1988) qu'un second horizon Bt en bandes (7.5 YR 4/6) s'est développé au sommet du massif dunaire apparemment déjà remanié (incorporation d'une charge en graviers schisteux) et qu'il a été disloqué lors de la mise en place des heads.

F. Scuvée et J. Vérague (1984) ont attribué la plage de base à l'Eemien (OIS 5e), la dune humifère et la dune ocre au Weichselien ancien (OIS 5b-5a). Les langues du podzol sont interprétées comme la trace «modérée » du coup de froid de la fin du Pléniglaciaire inférieur (Weichselien II (OIS 4 \& 3) et le complexe E2-3 est corrélé avec le Pléniglaciaire supérieur (OIS 2). E2-3 est corrélé avec le Pléniglaciaire supérieur (OIS 2). Ces interprétations positionneraient donc, selon Scuvée \& Vérague, l'industrie laminaire dans un contexte rapportable au Paléolithique supérieur. 
Apparemment, les paléosols (sols bruns lessivés en bandes, sols humifères) n'ont pas été observés en tant que tels et considérés comme des sédiments ruisselés pour l'analyse stratigraphique du site. Par ailleurs, l'état de conservation des vestiges organiques n'a suscité aucun questionnement alors que la malacofaune est bien préservée, mais que les os des grands mammifères s'avèrent très altérés. Initialement, une masse de sable calcaire avait été observée en placage sur les dépôts dunaires anciens mais avait été considérée comme Holocène par F. Scuvée et al. (1984). Il s'agit d'une loupe de dune calcaire à malacofaune qui a été réobservée en 1998 et datée par OSL (Folz, 2000 ; Cliquet et al., 2003).

Toutes ces observations effectuées au Rozel, ainsi que la lecture des autres coupes relevées dans ce secteur du Cotentin permettent de proposer une autre interprétation de la stratigraphie (Van Vliet-Lanoë, 1988) (fig. 4 et 8 ) :

- D1b/c - plage transgressive à gros galets et blocs, avec un petit sol humifère formé en phase finale ;

- D2a - head sableux suivi par une dune humifère ou une dune surmontée par un sol brun lessivé en bandes (D2b) ; la dune est probablement contemporaine du retrait postérieur au maximum marqué par D1 ;

- D2c - remaniement du sommet de la dune ocre et podzol humo-ferrique; sol d'habitat avec débris d'os et charbons ;

- D3 - grand massif dunaire régressif contemporain de la fin de D2 dont le sommet est remanié sous forme de head incorporant des blocs puis, développement d'un sol brun lessivé en bandes.

La séquence stratigraphique retrouvée est équivalente à celle de la côte nord du Cotentin, mais les massifs dunaires sont ici plus importants ; les épisodes de refroidissement qui, sur la côte nord voient la mise en place de heads, sont seulement caractérisés ici par le remaniement de faciès dunaire de retrait avec incorporation à leur sommet de débris rocheux en proximité de falaise.

\section{4 - LES OCCUPATIONS ANTHROPIQUES DE LA POINTE DU ROZEL (D.C.)}

Au moins deux niveaux d'occupation ont été mis en évidence dans les formations dunaires du Rozel (fig. 5) :

- le niveau Scuvée E2-E3 qui associe un paléosol à des restes de faune et des artefacts en silex

- le niveau Scuvée F2 de l'abri TR 67, sable pédogénéisé qui conserve des « lambeaux » de structures de combustion, des vestiges de faune consommée et des artefacts de silex et de quartz.

\section{2 - ANALYSE DU SITE D'HABITAT : L'APPORT DE LA TAPHONOMIE DU REMPLISSAGE DE « L'ABRI TR 67 » (D.C.)}

Plusieurs aspects de l'interprétation des niveaux d'occupation de l'abri proposée dans la monographie nous ont incité à tenter l'analyse taphonomique de son remplissage.

Nous reprendrons partiellement les éléments relatifs aux niveaux d'habitat développés par F. Scuvée (1984).

«Ces niveaux sont reconnaissables par l'existence de divers foyers, riches en cendres et charbons de bois, ainsi que par celle de silex taillés et os fossiles, déchets de consommation humaine.

$\mathrm{Au}$ fond de la grotte, nous notons la présence de deux lentilles chargées en charbons de bois et cendres. La première, entre $(8,20 \mathrm{~m})$ et $(8,50 \mathrm{~m}) \mathrm{NGF}$ (nivellement Scuvée \& Vérague), pourrait être la trace de la plus ancienne occupation des lieux, en supposant qu'il ne s'agit pas d'un amas de déchets rejetés là à partir d'un foyer situé plus en avant vers l'entrée (ce qui ne peut être démontré). Cette lentille serait antérieure au groupe des foyers, car, nettement plus basse altimétriquement, elle en est séparée par une coulée oblique de sable légèrement limoneux. Séparée de cette lentille par une mince couche de sable jaune clair, existe une modeste couche limoneuse, avec cendres et charbons de bois, qui parait interrompue par cette coulée, pan de foyer ou, encore une fois, rejet de déchets par balayage.

De $(8,90 \mathrm{~m})$ à $(9,20 \mathrm{~m}) \mathrm{NGF}$, se rencontre une seconde lentille de même nature que la première, séparée de celle-ci par près d'un demi mètre de sable jaune clair déposé en forme de lentilles obliques, différemment chargées de matériaux naturels divers (plaquettes et limon).

A l'entrée de l'abri, nous avons mis en évidence trois groupes de niveaux charbonneux ; ils sont tronqués par l'éboulement partiel de la terrasse, mais il nous a été possible de retrouver, sur la pente, glissés en bloc, des pans entiers de ces groupes de foyers...

Les sols d'habitat proprement dits sont extrêmement complexes et à peu près inextricablement mêlés; il est cependant possible d'affirmer que, étant donné les superpositions très étroites, en général, des divers foyers, il y eut cinq stades d'occupation principaux, si nous acceptons l'hypothèse de l'alternance d'établissement des foyers, tantôt à l'entrée, tantôt au fond de la grotte. Mais ces cinq groupes peuvent également se subdiviser, très souvent, chacun, en plusieurs sous-stades secondaires, avec, entre eux, de courtes périodes d'abandon pendant lesquelles de minces couches de sable éolien ne contenant ni cendres, ni charbons de bois, se seraient déposées.

Tous les vestiges archéologiques ont été rencontrés dans les zones à cendres et charbons de bois, ainsi que, mais en beaucoup moins forte densité, dans les zones chargées en plaquettes de schiste. D'anciens sols d'occupation ont très certainement existé, marqués, en théorie, par les dépôts superficiels de témoins 


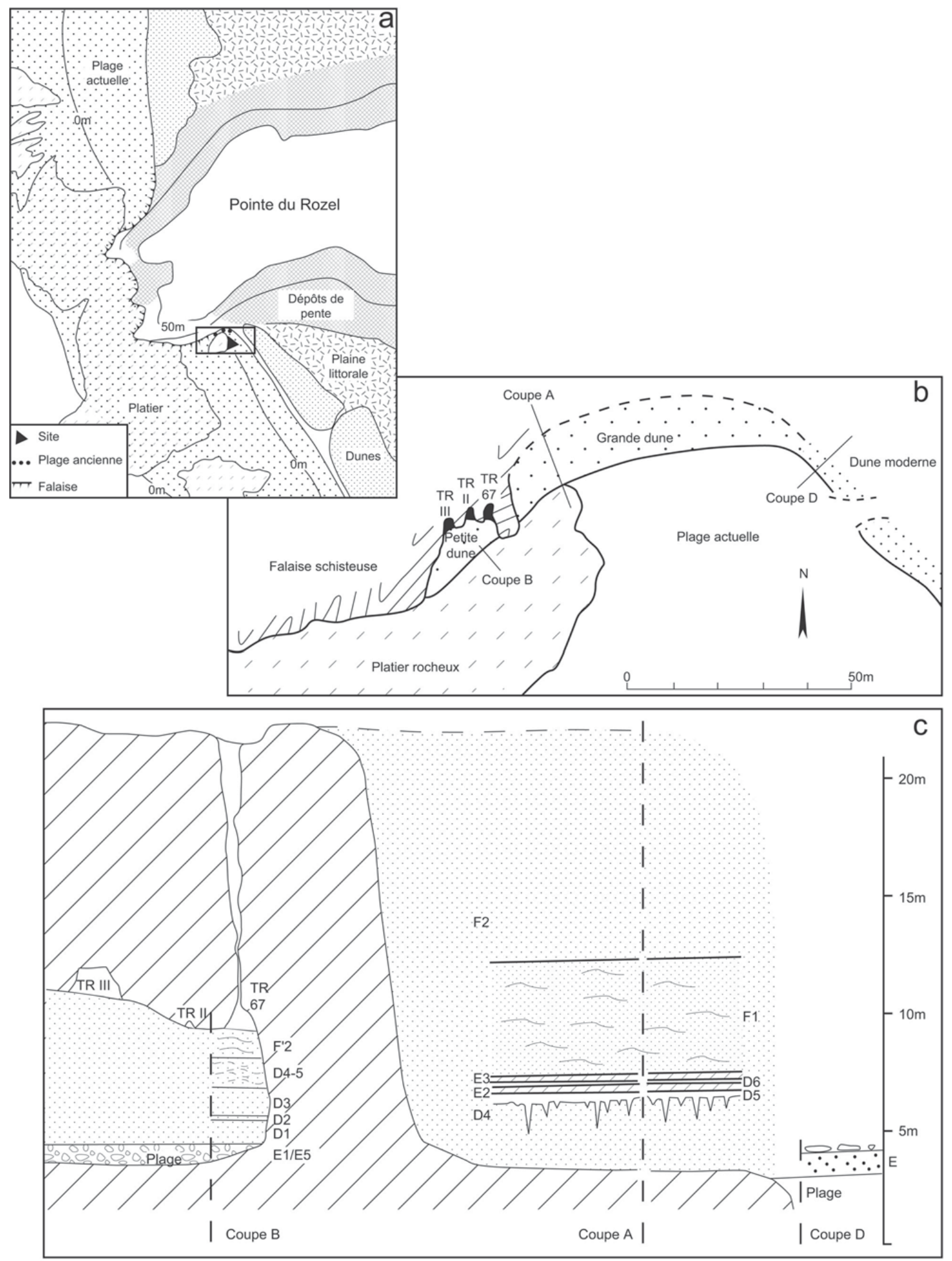

Fig. 5 : Localisation du site et enregistrement sédimentaire des abris et de la Grande Dune (d'après Scuvée \& Vérague, 1984). Fig. 5: Location of site and sedimentary evaluation of the rock shelters and la Grand Dune (after Scuvée \& Vérague, 1984). 
archéologiques ; mais ils sont, dans la réalité, très difficilement discernables à cause de la nature même du terrain, sable léger très aisément perturbé par le moindre piétinement des occupants. Aussi, l'examen altimétrique minutieux de la disposition des divers objets ne nous a guère apporté d'enseignements sur ce sujet ; les seuls repères relativement fermes restent les diverses lentilles et amas chargés de traces charbonneuses, ainsi que les variations dans la nature des sédiments.

La grande complexité des sols d'occupation et des foyers, leur grand nombre séparés par de courtes périodes d'abandon ou d'extinction des feux matérialisées par les fines couches de sable stérile intercalaires, militent contre l'hypothèse qu'il s'agirait d'un habitat permanent et de longue durée. Au contraire, il nous paraît comme évident qu'il s'agit d'un abri temporaire pour des chasseurs, d'ailleurs assez peu nombreux à cause de l'exiguïté de l'excavation, encore que nous ignorerons toujours si ces chasseurs ne disposaient pas d'abris en forme d'appentis ou tentes appuyés contre le flanc de la roche ou du pilier voisin. La construction d'un foyer organisé en dalles de schiste ne peut être considérée comme un indice de permanence, ni même d'une longue durée de séjour, car cette structure, du reste très rudimentaire, peut être montée en très peu de temps ; le petit nombre relatif des artefacts et des restes d'alimentation carnée ne peut non plus être un indice probant, à cause de l'absence d'une partie inappréciable de la terrasse qui exista devant la grotte. Dans le groupe de foyers nous avons rencontré au moins une structure lithique organisée d'aire de feu constituée par un dallage de plaques de schiste et une murette latérale. Les plaques formant le fond de l'aire étaient disposées d'une manière grossièrement jointive, à l'horizontale se relevant légèrement sur trois bords. Le bord ouest présentait plusieurs dalles de plus grandes dimensions, posées de chant et appuyées sur deux assez grandes dalles épaisses posées à plat, ménageant un étroit passage le long de la paroi rocheuse. Cette fosse, interrompue vers la terrasse de la grotte par l'effondrement de celle-ci, contenait plusieurs strates charbonneuses (au moins trois), dont la dernière, supérieure, était creusée d'une fosse de forme ovale (L. : 0,$80 ; 1 .: 0,27$; profondeur : $0,15 \mathrm{~m}$ ) aux flancs sub-verticaux dont le remplissage en sable avec plaquettes de schiste, était stérile d'artefact et débris organique ».

L'analyse des rapports de fouilles et de la monographie témoigne d'importants problèmes taphonomiques.

Si les observations conduites par F. Scuvée ont porté sur l'ensemble de la plateforme adossée à la falaise de schiste, seul l'abri TR 67 a livré des vestiges sur une aire très limitée (2,5 mètres de largeur) mais, sur une grande épaisseur (environ 1,60 mètre).

La complexité de lecture des couches d'habitat est attestée dans le rapport de 1969 : «Nous ne ferons pas intervenir ici la stratigraphie des trouvailles, trop complexe (p. 9)... Les foyers superposés rencontrés ne sont pas exactement situés sur le même emplacement et ne semblent pas non plus avoir eu la même importance. Toutefois, les témoins rencontrés dans leur masse ne semblent guère être différents au point de vue typologique, zoologique, quantitatif et qualitatif, tout au moins au premier examen ( $p .10$ et 11)...».

Des observations similaires avaient déjà été formulées dans le rapport (1967/1968), mais surtout le fouilleur indique que «toutes les couches rencontrées sont très fortement inclinées d'ouest en est, allant jusque $45^{\circ}$ d'angle et plus ( $\left.p .3\right)$ ) et que «les niveaux sont détruits le long de la paroi est, soit par un terrier, soit par une érosion (p. 3)».

Ces observations attestent d'un basculement de la couche d'habitat vers l'est après déstructuration du substrat sableux sur la bordure est du promontoire (terrier ou érosion imputable à un phénomène de suffosion par les eaux de ruissellement. Cette dynamique liée aux contraintes exercées par la ségrégation de glace dans des sols n'est pas sans évoquer ce qui a pu être reconnu à la Cotte Saint-Brelade (Jersey ; Callow et al., 1986) et sur le site du Fort à Saint-Vaast-la-Hougue (Fosse et al., 1986).

Le recouvrement et la discontinuité des sols charbonneux, des structures de combustion et des niveaux de plaquettes de schistes desquamées sous-jacentes évoquent le « glissement de cartes à jouer qui viennent se superposer».

Seul le recours à la technique des raccords de pièces lithiques débitées était susceptible d'apporter des éléments de réponse.

Si les artefacts ont été enregistrés par niveaux archéologiques, donc en 3 dimensions lors de la première campagne de sondages (1967/1968), seules les coordonnées planimétriques ont été relevées pendant la seconde (1969). Les plans indiquent que les artefacts lithiques et osseux ont été collectés entre la côte 8,88 mètres et la cote 8,20 mètres. Nous avons défini une côte moyenne à 8,60 mètres pour les artefacts issus de la seconde campagne, ce dans le but de matérialiser en 3 dimensions les différents raccords de pièces lithiques.

La représentation graphique des raccords lithiques atteste de l'existence d'un même niveau archéologique, dilaté sur environ 1.60 mètre, ce que corrobore l'analyse technique, technologique et typologique du mobilier (cf. infra) (fig. 6).

Il est donc fort probable que le niveau archéologique ait occupé l'ensemble de la cavité définie par TR67, TR. II et TR. III, soit un espace au moins large de 11 mètres (?)

\section{3 - DATATIONS OSL (N.M.)}

\section{1 - ÉCHANTILLONNAGE, MESURE DES DÉBITS DE DOSE ET DES PALÉODOSES}

Quatre échantillons ont été prélevés au Rozel (Folz, 2000) :

LR1 provient de la base de la dune sableuse (D1d1) située à une trentaine de centimètres au-dessus de la plage fossile éémienne. LR2 est situé juste en dessous du premier sol d'occupation (D2c). Les deux autres 


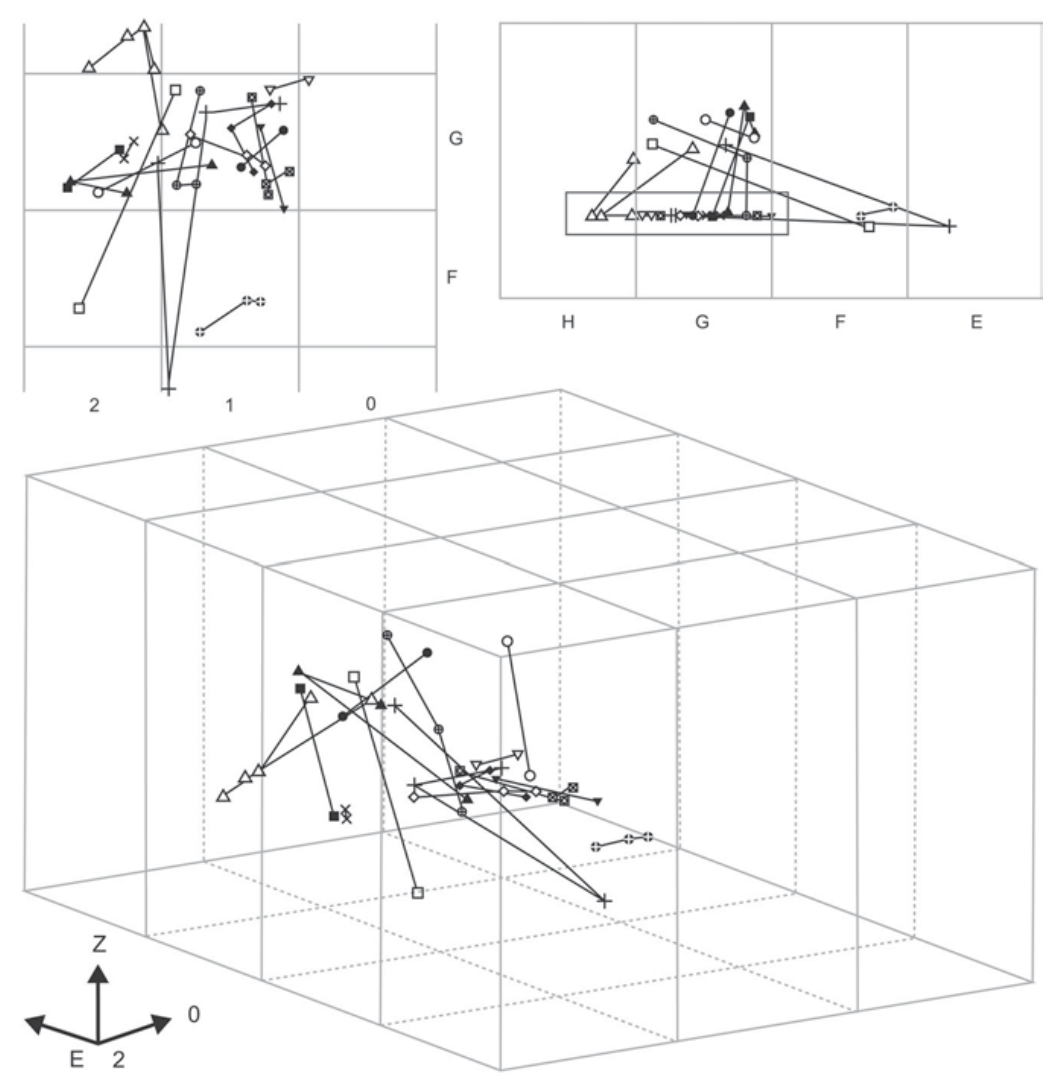

Fig. 6 : Raccords d'artefacts vus de dessus, de face et représentation 3D des raccords d'artefacts lithiques (DAO : F. Cliquet). Fig. 6: Conjoining of the artefacts: top view, front view and $3 D$ view (CAD: F. Cliquet).

échantillons proviennent d'un complexe, non référencé antérieurement, repéré lors de l'opération de prélèvement des échantillons : LR3 est associé à un niveau où quelques silex ont été découverts, surmonté d'un second niveau contenant des coquilles (LR4).

Les quatre échantillons sont des sables éoliens assez grossiers (peu de grains de diamètre inférieur à $200 \mu \mathrm{m})$, non carbonatés, à l'exception de LR4 du fait probable de la présence de coquilles.

Les mesures de débits de dose ont été réalisées sur le terrain au moyen d'un dosimètre, laissé 8 mois environ au niveau du prélèvement LR2, et d'un spectromètre portable qui a permis de faire une mesure in situ, associée à chaque prélèvement. L'analyse des spectres gamma a cependant montré que la radioactivité était relativement basse au Rozel, quel que soit le niveau étudié : les débits de dose, rapportés à un environnement sec, s'étalent en effet de 470 à $330 \mu \mathrm{Gy} / \mathrm{an}$ et sont de fait sensiblement inférieurs au débit enregistré par le dosimètre : $531 \pm 50 \mu \mathrm{Gy} / \mathrm{an}$. Cette différence peut s'expliquer par le faible taux de comptage auquel a été soumis le spectromètre de terrain et par là même, à la faible statistique obtenue. Au contraire, la dose mesurée par le dosimètre est certainement plus fiable puisque ce dernier est resté enfoui plusieurs mois, rendant ainsi la dose enregistrée parfaitement mesurable. Malgré l'unicité de cette mesure, cette dose a été utilisée dans le calcul des âges rapportés ci-dessous, en considérant une teneur en eau moyenne de $[25 \pm 10] \%$ et en estimant la dose cosmique à partir de l'épaisseur actuelle de la dune. Du fait de la puissance de ce massif dunaire, cette dose cosmique ne représente toutefois qu'une faible fraction de la dose annuelle.

Des mesures de spectrométrie gamma à bas bruit de fond ont aussi été réalisées au laboratoire sur des échantillons d'une centaine de grammes environ pour estimer l'état d'équilibre radioactif des sédiments étudiés. Aucun déséquilibre significatif n'a été détecté mais la dose gamma déduite des teneurs en radioéléments (uranium, thorium et potassium) est là encore inférieure pour LR2 $(299 \pm 23 \mu \mathrm{Gy} / \mathrm{an})$ à celle mesurée par le dosimètre. Cette différence reste à interpréter même si l'échantillon analysé peut, du fait de sa très faible masse et donc de son faible volume par rapport à celui perçu par le dosimètre (qui correspond à une sphère de $30 \mathrm{~cm}$ de rayon environ), n'être que peu représentatif de l'environnement du prélèvement LR2.

Des analyses par activation neutronique portant sur les grains de quartz dont les signaux de luminescence ont été étudiés, ont montré que les teneurs des quartz en radioéléments étaient faibles ( $\mathrm{U}: 0.13 \mathrm{ppm}$, Th: $0.32 \mathrm{ppm}$ pour LR4 et U : $0.12 \mathrm{ppm}$, Th : $0.45 \mathrm{ppm}$ pour LR1) ce qui conduit à une dose interne inférieure à $2 \%$ de la dose annuelle totale.

Pour la détermination des paléodoses, une technique d'aliquote unique a été utilisée. Chaque partie « aliquote », 
composée de plusieurs centaines de grains de quartz déposés sur un disque d'acier, a subi plusieurs cycles de mesure comprenant chacun une irradiation, suivie d'une chauffe de 10 secondes à $260{ }^{\circ} \mathrm{C}$ avant que le signal de luminescence ne soit mesuré par stimulation optique. Selon les échantillons, entre 43 et 58 fractions aliquotes ont ainsi été analysées et ont chacune fourni une valeur de paléodose. La figure 7 montre, pour chaque échantillon, la distribution des paléodoses obtenues normalisées par rapport à la moyenne. On constate une relative dispersion dont l'ampleur dépend des échantillons. Celle-ci est en partie explicable par des variations dosimétriques à l'échelle millimétrique mais aussi, probablement, par une remise à zéro différentielle du signal de luminescence des grains de quartz, au moment de leur enfouissement. Pour lisser ces variations, les paléodoses moyennes ont été calculées
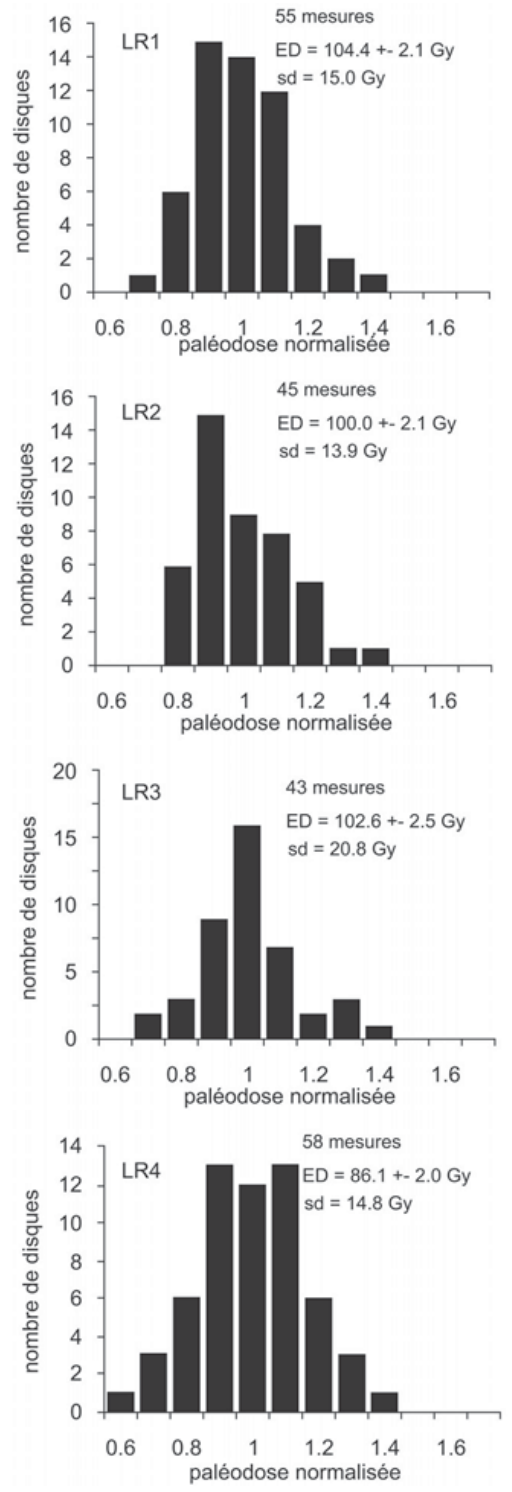

Fig. 7 : Distributions des paléodoses mesurées sur les échantillons du Rozel (d'après Folz, 2000).

Fig. 7: Palaeodoses and ages from the samples from Le Rozel (after Folz, 2000).

\begin{tabular}{|lrcc|}
\hline Echantillon (unité) & \multicolumn{1}{c}{$\begin{array}{c}\text { Paléodose } \\
\text { (Gy) }\end{array}$} & $\begin{array}{c}\text { Dose } \\
\text { annuelle } \\
\text { (mGy/an) }\end{array}$ & Age(ka) \\
LR4 (D3) & $86.1 \pm 2.1$ & $0.84 \pm 0.07$ & $102 \pm 10$ \\
LR3 (loupe glissée) & $102.6 \pm 2.4$ & $0.91 \pm 0.08$ & $113 \pm 11$ \\
LR2 (D2c) & $100.0 \pm 2.3$ & $0.93 \pm 0.08$ & $107 \pm 10$ \\
LR1 (D1c) & $104.4 \pm 2.3$ & $0.91 \pm 0.08$ & $115 \pm 11$ \\
\hline
\end{tabular}

Tab. 1 : Données OSL obtenues sur des échantillons de sables de la Grande Dune.

Tab. 1: OSL dates obtained on sand sample from La Grande Dune.

et sont portées dans le tableau 1, ainsi que les âges déduits.

\section{2 - RÉSULTATS ET DISCUSSIONS}

Les résultats obtenus avec la technique de l'aliquote unique, composée de multi-grains, sont statistiquement indiscernables pour les quatre échantillons. Ils situent dans la première moitié du stade isotopique 5 le dépôt des couches D1c à D3a2 et permettent de rejeter l'attribution de l'industrie lithique retrouvée dans l'abri au début du Paléolithique supérieur, initialement proposée par Scuvée \& Vérague (1984). Ils corroborent aussi l'appartenance de la série lithique du Rozel au groupe des industries laminaires de la phase récente du Paléolithique moyen, datées entre 110 et $70 \mathrm{ka}$ (Van Vliet-Lanoë et al., 1993).

\section{4 - LES DONNEES ENVIRONNEMENTALES}

\section{1 - RÉVISION DE LA STRATIGRAPHIE DU SITE (B. V. V.-L.)}

\section{Ensemble Petite Dune et Abri TR. 67}

A l'ouest du site, à la base du pied de falaise rocheuse, sur un platier assez irrégulier, exactement en contrebas des abris sous-roche, un premier affleurement existe. Il s'agit de la « Petite Dune » de Scuvée \& Vérague (1984). On y observe (fig. 8) :

- D1a - Une plage à galets de schistes assez gros, posés à plat avec une matrice sablo-humifère, légèrement limoneuse, riche en éclats schisteux (dynamique glacielle et contribution de dépôts de pente locaux). Un petit niveau humifère y est préservé (D1a2).

- D1b - Une récurrence de galets et de schistes repose en conformité sur ce premier niveau de plage.

Sur cette plage repose un sable dunaire moyen, illuvié en bandes d'argile brun ocre, enrichi en plaquettes de schistes (D2a).

Le reste de la stratigraphie observée par Scuvée \& Vérague (1984) n'est plus accessible. 


\section{Au niveau de la « Grande Dune »}

\section{"La plage eemienne"}

- D0 - Au sud du site, un niveau sableux subsiste sous la plage (observation Scuvée \& Vérague, 1984 ; Van Vliet-Lanoë, 1988). Il a été observé en pied de falaise en 1998, au sud du site archéologique proprement dit, lors d'un sondage effectué sous la « dune principale », donc sous D1a. Il s'agit d'un sable de bas d'estran.

- D1a - Ce sable est coiffé par un cordon de galets ronds, gréseux avec quelques silex, bien émoussés, situé au niveau des hautes mers actuelles $(+4 \mathrm{~m}$. NGF). La composition pétrographique dénote une dérive littorale importante en provenance du sud.

- D1b - Ce sol est recouvert par $50 \mathrm{~cm}$ de galets de schiste vert du Rozel posés à plats, avec une matrice sableuse, dont la composition pétrographique atteste d'un remaniement de dépôts de pente locaux, comme c'est le cas aujourd'hui (érosion).

\section{« La dune inférieure »}

- D1 c - Cet ensemble est recouvert par $80 \mathrm{~cm}$ de sables, parfois limoneux, compactés (zone de swash, surcote de tempête) correspondant à un estran moyen sableux (+ 6 m NGF). Au sommet de cette unité une première génération de fentes a été observée par Scuvée \& Vérague (1984). Il s'agit d'après les photographies publiées, d'incisions de ruissellement dans un sable compact. Cette incision atteste d'un abaissement du niveau marin après un niveau de surcote de tempête. Il a été daté de $115 \pm$ 11 ka (LR.1, Folz, 2000). Cette plage est probablement péné-contemporaine d'un niveau de sable dunaire, encore calcaire avec coquilles (déterminations D. Keen) ; qui a formé un placage épais le long de la falaise et qui sera repris postérieurement dans D3 par une loupe de glissement datée de $113 \pm 11$ ka (LR.3 ; Folz, 2000)

- D1d - 1,50 m de sable dunaire, décarbonaté. Il a été daté de $107 \pm 10$ ka (LR2 ; Folz 2000).

Il est stabilisé par un petit sol humifère, D2a1, postérieurement illuvié d'argiles (environ $50 \mathrm{~cm}$ ).

\section{«Les niveaux d'occupation »}

D2a2 - Ce sol est recouvert par un head sableux humifère incluant des blocs de schistes flués, également illuviés d'argiles et de charbons d'if (détermination H. Seignac).

D2b1 - Une stabilisation longue permet la formation d'un horizon illuvial en bande (Bt) qui a été confondu avec une stratification par J.Vérague. L'illuviation argileuse pénètre les unités inférieures à partir de son sommet; il s'agit d'un sol tronqué.

D2b2 - Ce niveau correspond à un remaniement de sables par les sols d'habitats en contexte déjà froid : trois récurrences distinctes ont pu être observées dans la «grande dune » avec incorporation de gros blocs déplacés par cryoreptation (coiffe silteuse, élongation et position des clastes). C'est dans ce niveau que l'industrie laminaire a été observée dans l'abri fouillé.

D2c1 - Un sable à peine stratifié correspond à un nouveau remaniement de sables par les sols d'habitats. Des fragments d'os altérés (consistance pâteuse) y ont été observés. Son sommet est marqué par un alignement de plaquettes schisteuses. Il s'agit de la couche E2 de F. Scuvée, vraisemblablement dérivée du niveau principal d'habitat.

D2c21- A son sommet, un podzol humo-ferrique à glosses se développe. Cette unité correspond aux fentes de gel de J. Vérague. Il est ensuite cryoturbé (fentes de cryodessiccation) et flué. Un mince niveau sableux clair coiffé par un horizon sablo-graveleux humifère riche en charbon coiffe cet horizon (D2c22) : il s'agit de la couche Scuvée-E3. Les éléments fauniques ont été trouvés dans toutes les strates de cette unité (Cervus elaphus, Equus). Ces unités ne contiennent pas de coquilles préservées. En pied de falaise, ce matériel n'est pas illuvié, à la différence de ce qu'on observe en milieu de versant, où se remarque une illuviation brunrougeâtre en provenance du sommet de la dune supérieure. Les coquilles observées par F. Scuvée se situeraient dans le sable clair entre E2 et E3. Il s'agit peut être d'une première déstabilisation de la falaise, avant l'évènement suivant. Selon F. Scuvée, E2 et E3 appartiennent à la même couche et sont contemporains des niveaux archéologiques de TR67.

\section{« La dune supérieure »}

D3a correspond à un sable massif sans illuviation argileuse. Une déstabilisation de la falaise se produit au début, remaniant des éléments du corps dunaire initial (calcaire) (datation LR4 à $103 \pm 10$; LR3 à $113 \pm 11$ ka, Folz, 2000). Le remaniement a dû s'effectuer au début, par glissement en masse sans blanchiment du sable, puis par ruissellement de la surface effondrée permettant une concentration d'artefacts et un blanchiment modeste. Il est coiffé par D3b, un apport latéral de head sableux humifère recouvert de très grosses dalles.

Ce matériel est ensuite enfoui par D3c, un corps dunaire remanié par ruissellement, de plus en plus riche en blocs vers son sommet et incluant de très gros blocs en proximité de falaise. Il s'agit d'un sable assez fin, riche en éléments angulaires repris à partir de lœss et de colluvions.

D3d1 correspond à un dépôt de pente gravelosableux. Un horizon $\mathrm{B} 2 \mathrm{t}$ en bandes, D3d2 rubéfie sa partie sommitale, et, D3c en partant de la partie inférieure du dépôt de pente. Il est fauché par cryoreptation.

\section{Remarques}

1) La vidange de TR 67 vers la Grande Dune est morphologiquement impossible. La présence de heads humifères à charge charbonneuse au sein de D2-3, au-dessus du niveau d'habitat de l'abri sous-roche montre qu'un autre abri indépendant de TR 67, existait derrière la « Grande Dune » ou en bordure nord de celle-ci. Il est probablement à la source des remaniements observés. 


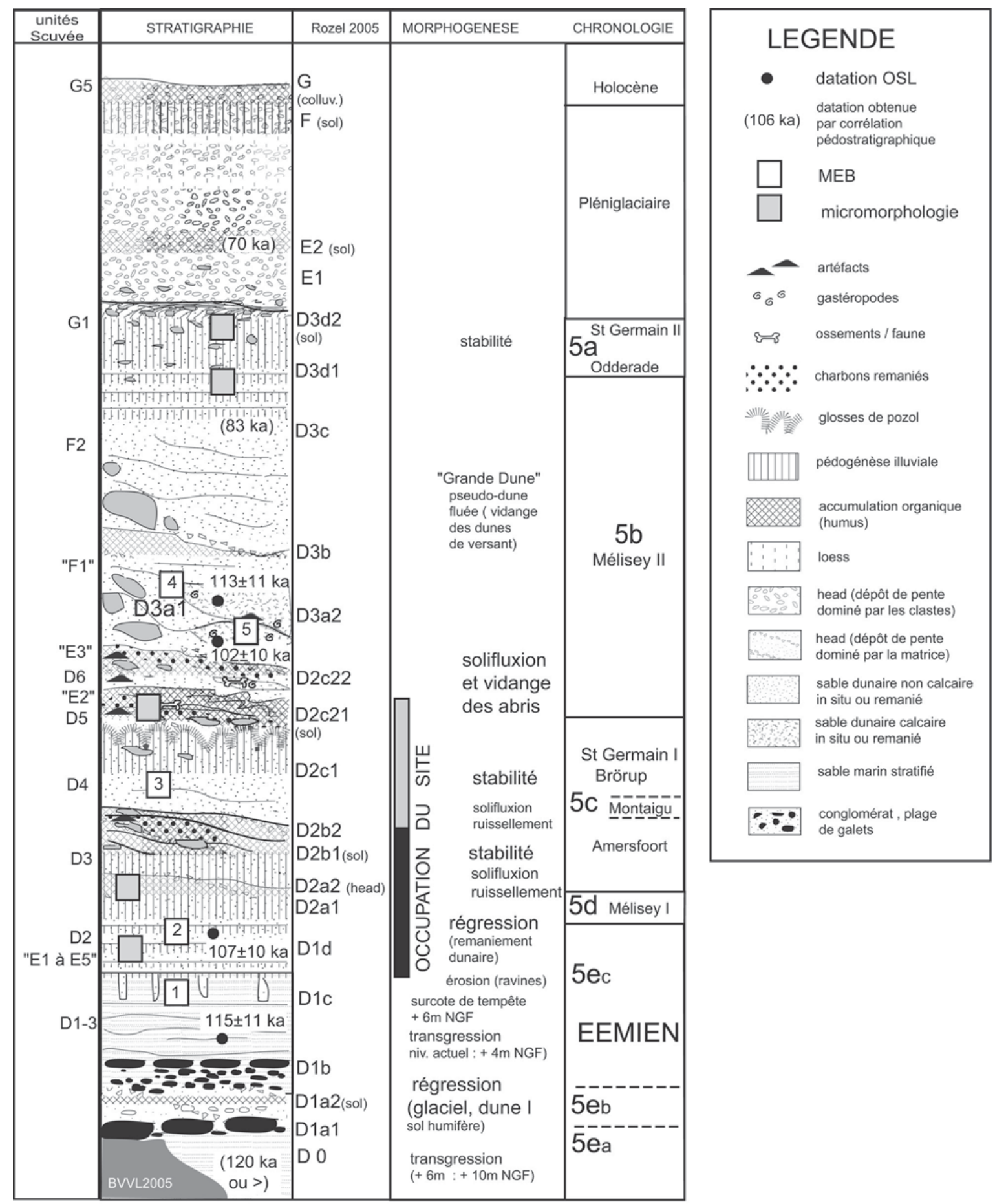

Fig. 8 : Enregistrement sédimentaire composite des abris et de la Grande Dune (Van Vliet-Lanoë, 2005). Fig. 8: Composite sedimentary log of the shelters and the Main Dune (Van Vliet-Lanoë 2005).

2) Le glissement de la dune calcaire a amené des reconcentrations en silex taillés au sommet de D2 / base de D3. Il est vraisemblable que la terrasse surmontant les abris a pu servir de promontoire utilisé par les hommes, autant que les abris. Ce dernier peut s'être effondré (gros blocs dans la base de D3).
3) Certains ossements (notamment en provenance de la collection Scuvée; D6 : mandibule de cerf élaphe) sont très frais et ont donc été remaniés à partir de la dune calcaire.

4) Le lessivage argileux rubéfiant au sommet de la "Grande Dune" atteste d'une neutralisation de l'acidité 
antérieure (décarbonation et acidolyse des os par la podzolisation) par incorporation de sables calcaires au sein de la masse décarbonatée (ZPC des colloïdes).

\section{« Les heads »}

E - La séquence passe ensuite à des dépôts de pente à blocs, les heads présentant un enregistrement assez voisin mais nettement moins dilaté que celui observé à Ecalgrain Nord au-dessus du pédocomplexe du Dernier Interglaciaire (Lautridou et al., 1986 ; Van VlietLanoë, 1988) : dépôts de solifluxion limono-sableux entrecoupés de paléosols humifères, puis à matrice plus limoneuse avec développement d'un sol lessivé à leur sommet. A l'aplomb de la "grande dune", ils sont relativement minces, puis s'épaississent vers le Sud, où ils sont recouverts par $1,50 \mathrm{~m}$. de colluvions schisteuses $(\mathbf{F})$ grises.

L'analyse micromorphologique (fig. 9) confirme les observations macroscopiques.

A) Horizon supérieur de D3c : dépôt de solifluxion à matrice limono-sableuse remaniant encore du sable éolien, mais également du limon et des grains anguleux. Noter l'illuviation obturante très pigmentée et litée, dénotant un contexte hydrologique contrasté.

B) Horizon D1c : sable dunaire grossier, proximal, riche en éléments émoussés, avec quelques résidus organiques (noirs) et une illuviation argileuse brun-jaune.

C) Horizon D2a1 : sable dunaire hétérométrique avec fragments anguleux, matières organiques décomposées et partiellement illuviées ainsi qu'une illuviation argileuse brun-jaune (en bas à gauche)
D) Horizon D2c2 : Bh du paléopodzol (matières organiques illuviées noires) sans illuviation postérieure, dans un sable dunaire hétérométrique, riche en fragments anguleux.

\section{2 - LES DUNES (M.M.)}

\section{Données granulométriques}

Un échantillonnage représentatif des unités nonpédogénéisées a été effectué, en général à proximité de leur base, afin de s'affranchir dans la mesure du possible de la composante illuviale des paléosols. Pour l'ensemble des échantillons, les résultats reprennent la médiane, les rapports entre les différentes classes granulométriques ainsi que les pourcentages de chaque classe granulométrique (fig.10).

Pour information, les classes granulométriques sont définies comme suit (classification ASTM) :

- Sables très grossiers : entre $1000 \mu \mathrm{m}$ et $2000 \mu \mathrm{m}$,

- Sables grossiers entre 500 et $1000 \mu \mathrm{m}$,

- Sables moyens : entre 250 et $500 \mu \mathrm{m}$,

- Sables fins : entre 125 et $250 \mu \mathrm{m}$,

- Sables très fins : entre 63 et $125 \mu \mathrm{m}$,

- Silts : entre 2 et $63 \mu \mathrm{m}$.

Les sables moyens constituent la classe granulométrique dominante pour tous les échantillons (médiane comprise entre 277.8 et $360.6 \mu \mathrm{m}$ ). La classe granulométrique "sables grossiers" est représentée dans tous les échantillons mais en faibles proportions. Les pourcentages des différentes classes granulométriques varient fortement selon les échantillons.

D1b (fig. 11-1) : L'analyse granulométrique montre une nette prédominance des sables moyens $(75 \%)$. Les
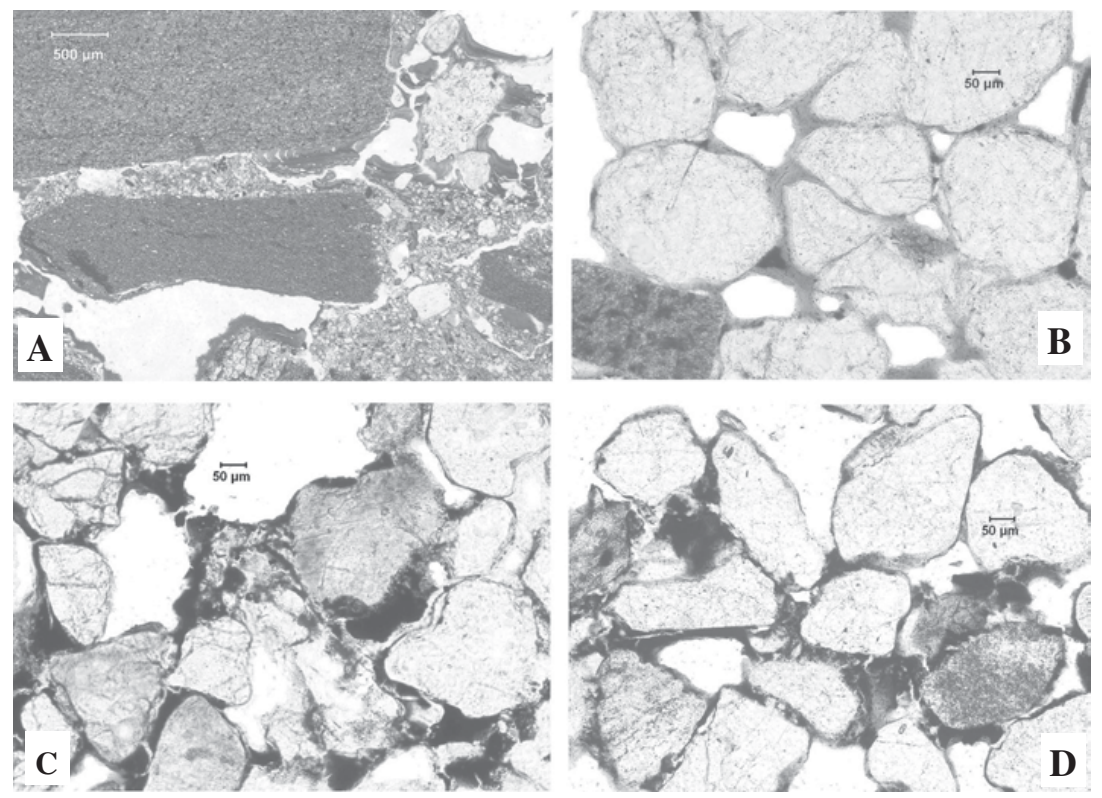

Fig. 9 : Analyse micromorphologique de D3c (en haut à gauche), de D1c (en haut à droite), de D2a1 (en bas à gauche) et de D2c2 (en bas à droite) (clichés B. Van Vliet-Lanoë).

Fig. 9: Micromorphological analysis of D3c (above left), Dc (above right), D2al (below left) and D2c2 (below right) (pictures B. Van Vliet-Lanoë). 


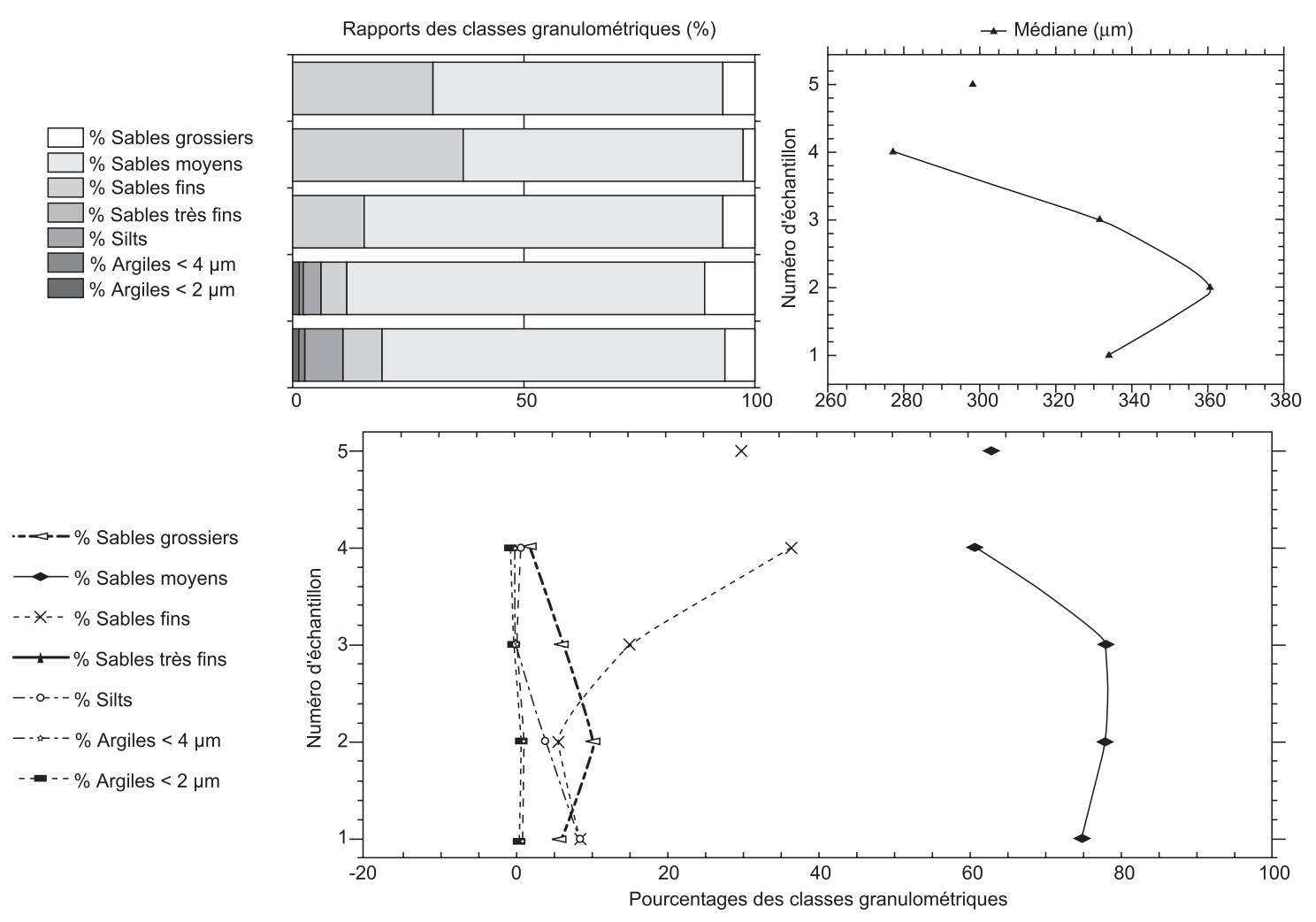

Fig. 10 : Rapports entre les différentes catégories granulométriques et pourcentages des catégories granulométriques des sables du Rozel (1 - base de D1b, 2 - base de D1d, 3 - base de D2b, 4 - D3b dune remaniée et 5 - D3 loupe de dune carbonatée.)

Fig. 10: Rozel grain sizes analysis.

sables grossiers, les sables fins et les silts sont en proportions sensiblement équivalentes (respectivement 6 , 8.1 et $9.7 \%)$.

La courbe est légèrement bimodale avec un petit pic à $50 \mu \mathrm{m}$ dénotant un apport vaseux. A noter la présence d'argiles illuviées, liées à la percolation du paléosol D2b1.

MEB : grains moyens à dominance arrondis avec forte dissolution superficielle. Bas d'estran. Echelle : $200 \mu \mathrm{m}$

D1d (fig. 11-2) : Les rapports granulométriques sont sensiblement équivalents à ceux de l'unité précédente. Elle se caractérise cependant par les plus fortes proportions en sables grossiers $(10.6 \%)$. Les sables fins, les silts et les argiles sont toujours présents mais en quantités moins importantes.

A noter, deux modes complémentaires à 10 et $50 \mu \mathrm{m}$.

MEB : Grains moyens à dominance arrondis avec quelques grains anguleux. Forte dissolution de la surface des quartz associée à la podzolisation. Les grains présentent parfois certaines traces de coups, des griffures et une fine pellicule illuviale. Remanié d'un haut d'estran. Echelle : $200 \mu \mathrm{m}$

D2b (fig. 11-3) : Cette unité se caractérise par les plus fortes proportions en sables moyens (78.2\%). A noter, la disparition des silts et des argiles qui ne sont plus représentés dans le reste de la série.

Le pourcentage en sables grossiers diminue.
MEB : Grains mêlant fins et moyens à dominance arrondis présentant un émoussé éolien. Sur certain grain, l'émoussé luisant est encore visible malgré l'altération par dissolution.

Sable dunaire remanié avec des apports de versant. Echelle : $500 \mu \mathrm{m}$

D3b (fig. 11-4) : dune supérieure : Diminution des fractions extrêmes, les plus faibles de la série $(63.3 \%)$ au profit des sables moyens.

MEB : Grains à dominance anguleuse avec très peu de grains arrondis, nombreux grains gélifractés (esquilles).

Faciès de versant remaniant de la dune et du limon. Echelle : $200 \mu \mathrm{m}$

D3 (fig. 11-5) : Loupe carbonatée (à coquille) : Trois classes granulométriques (sables grossiers, moyens et fins) sont présentes dans cette unité avec une dominance de sables moyens. Les pourcentages de sables fins sont parmi les plus forts de la série $(30.2 \%)$. Il en est de même pour les sables grossiers $(6.5 \%)$.

MEB : Grains à dominance arrondie avec la présence cependant de quelques grains anguleux, dérivés d'apport de versant ou fluviatile. Echelle : $200 \mu \mathrm{m}$

L'analyse des formations sableuses met en évidence plusieurs éléments. Les sables d'estran observés au MEB à la base sont nettement plus grossiers que les formations dunaires sus-jacentes. Il existe manifestement un tri granulométrique avec l'altitude du dépôt, ce qui ne dénote pas des vents extrêmement violents. Le 

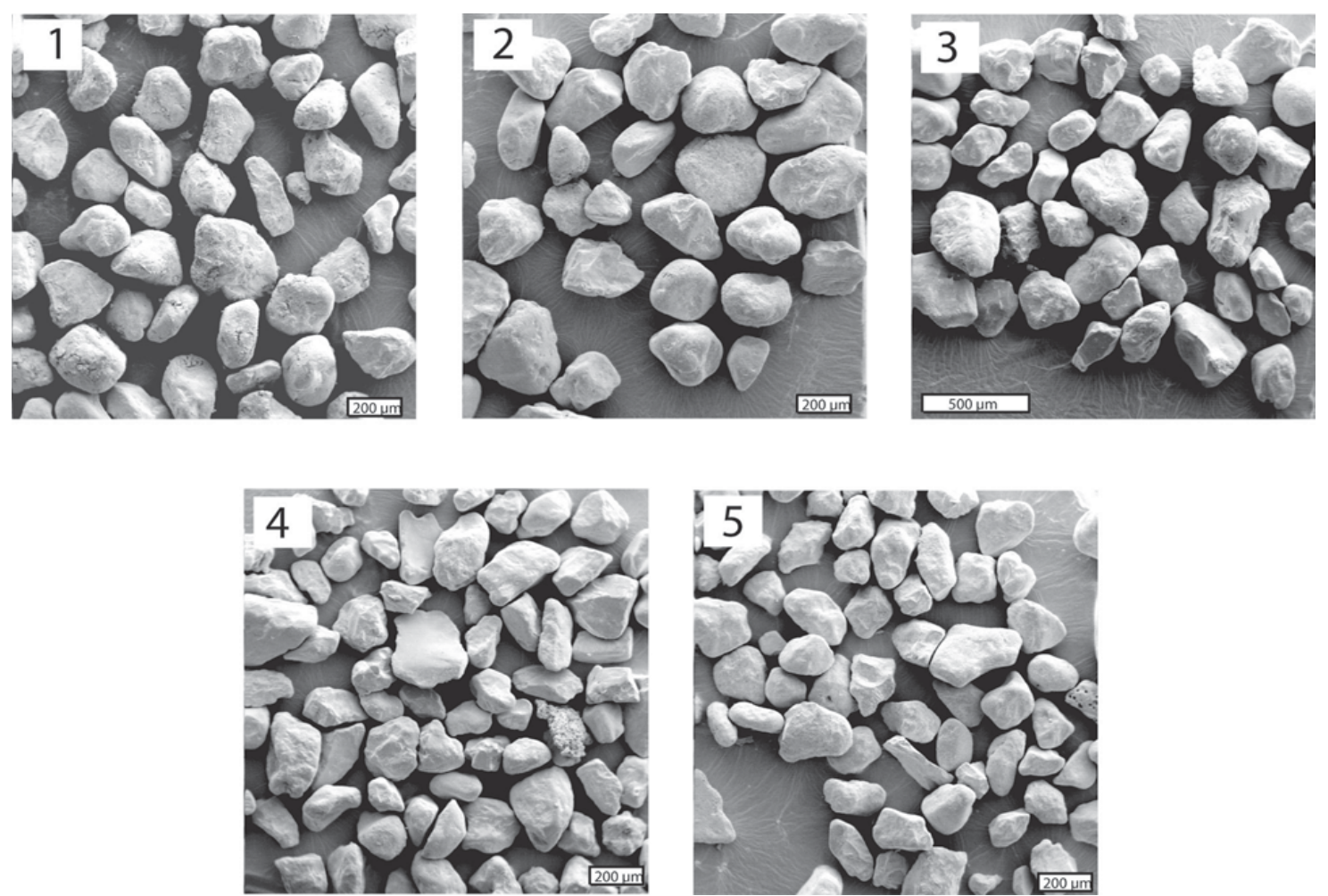

Fig. 11 : Analyse au MEB des sables (1) de D1b, (2) de D1d, (3) de D2b, (4) D3b dune remaniée et (5) de D3 loupe de dune carbonatée (clichés M. Meurisse).

Fig. 11: Electron microscope analyses of the sands of (1) - DIb, (2) DId, (3) D2b, (4) D3b reworked dune and (5) D3 slumped calcareous dune (picture M. Meurisse).

matériel de la dune calcaire commence à contenir des grains anguleux dérivés des formations continentales. Leur proportion croît pour les unités supérieures du complexe dunaire, dénotant une contribution croissante des apports fluviatiles à la côte en réponse à une érosion croissante des sols et formations superficielles périglaciaires à l'intérieur des terres comme nous pouvons aussi le constater pour l'Holocène (Meurisse $e t$ al., 2004). Des figures de dissolution sont marquées pour les éléments supérieurs en relation avec la podzolisation des sols, à l'extérieur de l'abri sous roche. Les analyses granulométriques du sable montrent que les unités D1 sont littorales avec un apport vaseux (courbe bimodale) sensible même dans les faciès dunaires. Les unités supérieures ont toutes un très bon classement et résultent du mélange de deux stocks éoliens (dune I, calcaire à l'origine et dune 2). Lors de la mise en place de D2 et D3, ces matériaux plaqués contre la falaise et sur le versant ont été repris par glissement ou solifluxion. Le ruissellement évoqué par J. Vérague aurait induit un classement différent des particules.

\section{3 - LE COUVERT VÉGÉTAL : L'ANTHRACOLOGIE (H. S.)}

La détermination des charbons collectés dans le sédiment sable humifère D2a2 montre la présence de feuillu et d'if (Taxus) (fig. 12 ; détermination H. Seignac, photographies M. Meurisse et B. Van Vliet-Lanöe). Si l'if préfère les sols profonds riches en humus, il tolère les sols rocheux à condition qu'ils soient fissurés. Une espèce actuelle irlandaise croit sur des versants d'éboulis secs et calcaires. Sa présence au Rozel n'est donc pas insolite. La présence de charbon d'if au Rozel n'est peut-être pas anodine. En effet, l'if est un bois dur, très résistant et élastique, à grain fin, donc d'un accès modéré. Rappelons seulement que cette essence a été utilisée, outre comme combustible, comme support d'arme de hast pour la chasse au paléolithique ancien et moyen (Clacton-on-sea, Grande-Bretagne ; Lehringen, Allemagne).

\section{5 - LA FAUNE (D.C., D. K. \& P. A.)}

L'ambiance environnementale ne peut être appréhendée, en dehors de l'analyse pédo-stratigraphique, que par la faune malacologique et les vertébrés.

\section{1 - LES MOLLUSQUES}

\subsection{1 - Observations anciennes (D.C.)}

Les gastéropodes prélevés durant les investigations menées entre 1967 et 1969 sont issus de la cavité TR. III, donc du remplissage de sable. Les déterminations ont été effectuées par J.-J. Puisségur (Scuvée \& Vérague, 1984 ; tab. 2 et 3 ). 
Feuillu
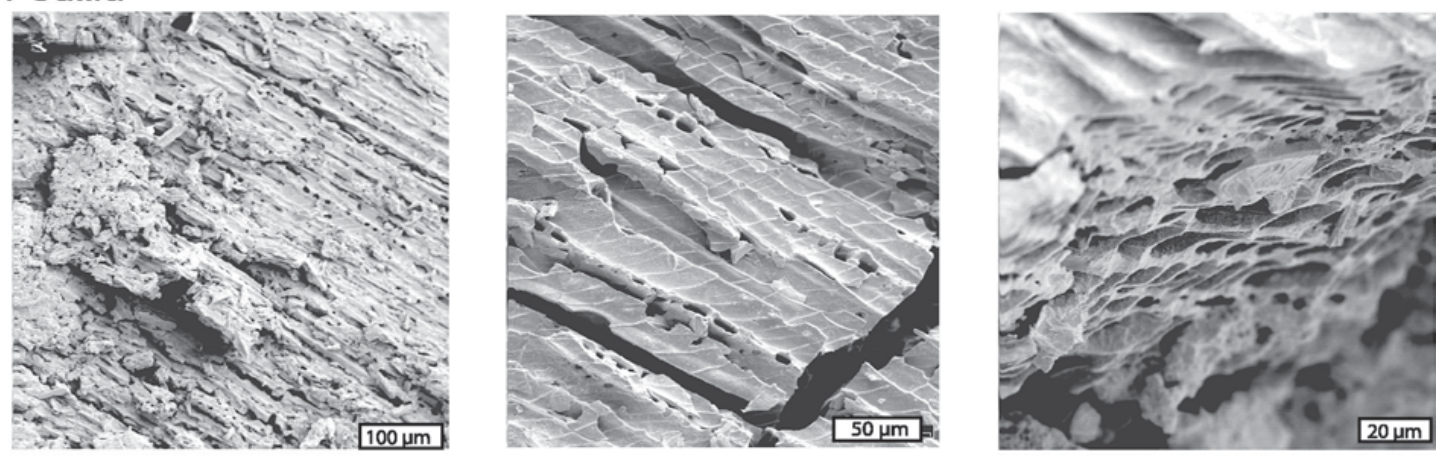

\section{If (Taxus)}
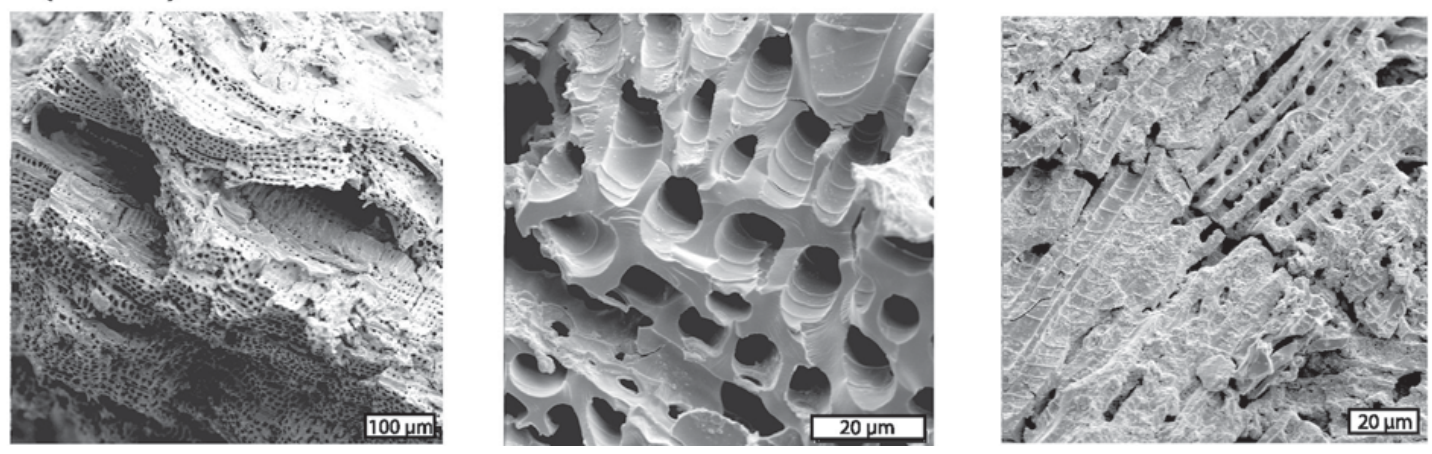

Fig. 12 : Charbons de feuillus et d'if (Taxus) de la couche D2a2 de B. V V-L. 2005 (clichés MEB M. Meurisse et B. Van Vliet-Lanoë). Fig. 12: Deciduous charcoal and Taxus charcoal from layer D2a2 (B.VV-L 2005)(SEM: M. Meurisse \& B. Van Vliet-Lanoë).

Ces éléments ne sont donnés qu'à titre indicatif, les échantillons n'étant pas localisés dans l'espace sur les plans ou dans les rapports de fouilles.

Selon F. Scuvée, «les gastéropodes rencontrés confirment l'indication chronologique apportée par la grande dune : climat steppique très sec et très froid du Weichsélien III » (Scuvée \& Vérague, 1984).

Les seules indications publiées à propos du cortège malacologique concernent les mollusques de la « Petite dune » (abri TR III, tab.1), et de la « Grande dune », sans indication de couche : «Il nous faut tenir compte des microclimats forts différents entre les divers lieux de prélèvement des témoins; par exemple, les gastéropodes prélevés dans la petite dune furent toujours dans des grottes, alors que ceux provenant de la «Grande dune » se trouvaient sur des sols exposés aux aléas climatiques » (Scuvée \& Vérague, 1984).

La liste publiée par Scuvée \& Vérague (1984) s'avère d'interprétation difficile eu égard, d'une part, à la faiblesse de l'échantillonnage (53 individus au

\begin{tabular}{|l|l|c|c|c|c|c|}
\hline Espèce & \multicolumn{1}{|c|}{ échantillons } & TR.13 & TR.16 & TR.53 & TR.61 & Total \\
\hline Semi forestière & Cepea nemoralis L. & & & & 1 & $\mathbf{1}$ \\
\hline & Cepea sp.. & & & & 1 & $\mathbf{1}$ \\
\hline Steppique & Candidula rugosiucula MICHAUD & & & & 8 & $\mathbf{8}$ \\
\hline & Helicella cf. striata MULLER & & & & 3 & $\mathbf{3}$ \\
\hline & Helicella sp. & 10 & 3 & 4 & 10 & $\mathbf{2 7}$ \\
\hline De terrain découvert & Pupilla muscorum L. & & & 1 & & $\mathbf{1}$ \\
\hline & Pupilla sp. & & 1 & & 2 & $\mathbf{3}$ \\
\hline Mésophile & Trichia hispida L & & 1 & 1 & & $\mathbf{2}$ \\
\hline & Clausilia sp. & & & & 1 & $\mathbf{3}$ \\
\hline & Vitrina major FER & & & & 2 \\
\hline & Oxychilus cellarius MULLER & 2 & & & & $\mathbf{2}$ \\
\hline & & & & & & $\mathbf{2}$ \\
\hline Total & & $\mathbf{1 2}$ & $\mathbf{7}$ & $\mathbf{6}$ & $\mathbf{2 8}$ & $\mathbf{5 3}$ \\
\hline
\end{tabular}

Tab. 2 : Espèces de mollusques par échantillon (déterminations J.-J. Puisségur). Tab. 2: Molluscan species from each sample (determined by J.J. Puisségur). 
total), d'autre part à l'abondance de Helicella sp., qui n'est pas un marqueur spécifique selon Kerney et Cameron (1979). En effet, ces derniers précisent que cinq espèces de Helicella vivent dans l'ouest de l'Europe en contexte de "prairie sèche" à "ombragé sous troncs d'arbres" et "prairie humide". Ainsi l'interprétation proposée par Scuvée \& Vérague selon laquelle cet assemblage vivait sous « un climat steppique très sec et froid » ne correspond pas à la liste d'espèces communiquée. Une modification taxonomique existe entre les expertises de J.J. Puisségur et celle de D. Keen : Vitrina major s'appelle maintenant Phecolimax major (Férussac) (voir également les données non numériques de Puisségur) ; l'espèce répertoriée comme Helicella ericetorum s'appelle actuellement Helicella itala.

La conclusion de Scuvée \& Vérague est donc erronée étant donné l'absence d'espèces de climat froid telles Columella columella ou Vertigo geyeri, et la présence d'espèces tempérées telles que Oxychilus cellarius ou Clausilia. Les deux espèces les plus représentées, (Candidula rigosiucula - aujourd'hui Candidula unfasciata (Poire) - et Helicella cf. striata - aujourd'hui Helicopsis striata (Muller) - ont toutes deux leur aire de répartition en France et en Allemagne et ne se trouvent ni en Scandinavie ni dans les Alpes (au-dessus de $2000 \mathrm{~m}$ ). Ce ne sont en aucun cas des espèces « steppiques » au sens «prairie froide sub-arctique ». Elles vivent en prairie mais pas dans des conditions très froides.

L'ensemble des gastéropodes collectés au Rozel et identifiés par J.-J. Puisségur est le suivant :

\begin{tabular}{|l|l|}
\hline Espèce forestière & Aegopinella pura ALDER* \\
\hline Espèce semi-forestière & $\begin{array}{l}\text { Cepea sp. } \\
\text { Cepea nemoralis L. }\end{array}$ \\
\hline Espèce steppique & Candidula rugosiuscula \\
& MICHAUD \\
& Helicella cf. striata MULLER \\
& Helicella sp. \\
& Helicella ericetorum MULLER * \\
\hline Espèce de terrain & Pupilla muscorum L. \\
découvert & Pupilla sp. \\
& Truncatellina cylindrica FER* * \\
& Vallonia costata MULLER * \\
& Vallonia pulchella MULLER* \\
\hline Espèce mésophile & Cochlicopa-lubrica MULLER* \\
& Punctum pygaeum DRAP. * \\
& Oxychilus sp. * \\
& Vitrea contracta WEST. * \\
& Trichia hispida L. \\
& Clausilia sp. \\
& Vitrina major FER. \\
& Oxychilus cellarius MULLER \\
\hline
\end{tabular}

Tab. 3 : Espèces de mollusques par biozones (déterminations J.-J. Puisségur).

Les espèces notées d'un astérisque proviennent de la «Grande dune » (sans indication de couche), ce qui devrait correspondre à la loupe de glissement.

Tab. 3: Molluscan species from each biozone determined by J.J. Puisségur).

Species with * were yielded in the main dune, probably in the carbonated dune slump.

\subsection{2 - Les données nouvelles (D.K.)}

Un prélèvement a été effectué en 1996 au niveau de la dune calcaire fluée, en parallèle avec celui de la datation TL. La faune collectée dans $1 \mathrm{~kg}$ de sédiment compte 480 individus correspondant à 15 taxons.

Le décompte de coquillages effectué comprend les apex des coquilles et les coquilles entières. Cette convention de décompte sous-estime la présence réelle d'espèces minoritaires si beaucoup de coquillages sont cassés. A la pointe du Rozel, il y avait des débris de Clausilia bidentata et Discus rotundatus en quantité considérable, impliquant que les décomptes fournis dans le tableau sont des valeurs minima.

Le grand nombre d'Helicella italia et des deux formes de Pupilla muscorum, environ $81 \%$ du cortège malacologique, suggère que la faune est en grande majorité une faune de prairie très probablement de milieu calcaire. D'autres espèces présentes, indicatrices de prairies, sont les espèces Truncatellina cylindri$c a$ et Vallonia. Quelques zones de prairie étaient humides, comme en témoignent Vallonia pulchella et Cochlicopa lubricella, mais l'existence de marais ou de marécages n'est pas attestée. La présence d'un seul spécimen d'Armiger crista (maintenant Gyraulus crista) est étrange. Cette espèce occupe les mares où abonde une végétation macrophytique et sa présence dans l'échantillon est difficile à prendre en compte à moins qu'elle ait été introduite sur le site, apportée par le vent depuis un marécage alentour. L'influence du sable apporté par le vent est suggérée par la présence en petit nombre de coquillages cassés de taxons marins dans l'échantillon (tab. 4).

Un petit nombre de coquilles tend à montrer un paysage différent de la prairie ouverte. La présence de Discus rotundatus et de Clausilia bidentata suggère l'existence d'habitats ombragés dans la zone. Habituellement ces 2 espèces indiquent la présence de forêt ou de broussailles mais Clausilia bidentata vit dans la quasi obscurité, sous des pierres lui permettant d'habiter dans des zones sans arbre telles que le Nord de l'Ecosse. Cependant la présence de Clausilia bidentata dans de telles zones reflète la répartition des arbres dans le cas de l'Holocène ancien et non la colonisation des zones toujours demeurées vierges. On peut noter que ces 2 indicateurs de zone ombragée ne représentent que $0.016 \%$ de la faune.

En résumé, l'environnement local à l'époque du dépôt était celui d'une prairie sur substrat calcaire mais avec peut-être des broussailles ou quelques forêts aux alentours. La variété d'habitats des espèces de prairie suggère la présence d'une prairie à la fois sèche sur la dune et plus humide en arrière du cordon littoral. Bien que la présence de débris de coquillages marins atteste un apport par le vent à partir des côtes voisines, leur petit nombre et leur état cassé montrent que soit la côte était éloignée, soit les vents de sable étaient limités.

En fait, la faune correspond à 15 taxons de contexte steppique et milieu humide frais. 


\begin{tabular}{|c|c|c|c|}
\hline \multicolumn{2}{|c|}{ Taxons de prairie et de milieu humide et frais } & \multicolumn{2}{|l|}{ Taxons marins } \\
\hline Cochlicopa lubricella (Porro) & 1 & Tricolia pullus (Linné) & 1 \\
\hline Cochlicopa $\mathrm{sp}$ & 1 & Rissoacea undet & 2 \\
\hline Truncatellina cylindrica (Férussac) & 17 & Mytilacea undet & 1 \\
\hline Vertigo pygmaea (Draparnaud) & 1 & Pectinacea undet & 1 \\
\hline Pupilla muscorum (Linné) & 65 & Cardiacea undet & 1 \\
\hline Pupilla muscorum bigranata Rossmâssler & 46 & Balanus sp & + \\
\hline Vallonia costata (Müller) & 18 & & + \\
\hline Vallonia pulchella (Müller) & 13 & & \\
\hline Vallonia enniensis (Gredler) & 6 & Total & 6 \\
\hline Vallonia sp & 14 & & \\
\hline Discus rotundatus (Müller) & 1 & & \\
\hline Aegopinella nitidula (Draparnaud) & 4 & & \\
\hline Limax sp & 1 & & \\
\hline Clausilia bidentata (Strôm) & 7 & & \\
\hline Cemuella virgata (da Costa) & 4 & & \\
\hline Helicella itala (Linné) & 280 & & \\
\hline Anniger crista (Linné) & 1 & & \\
\hline Total & 480 & & \\
\hline
\end{tabular}

Tab. 4 : Taxons marins, de prairies et de milieu humide et frais prélevés dans la Grande Dune (détermination D. Keen). Tab. 4: Temperate marine and land taxa from la Grande Dune (determined by D. Keen).

\subsection{3 - Environnement régional et climat selon les données de la malacofaune (D.K.)}

Les mollusques peuvent être utilisés comme indicateurs climatiques de deux manières. Comme le nombre d'espèces décline rapidement dans les zones du Nord de l'Europe, le nombre d'espèces présentes est souvent un bon indicateur du climat lors du dépôt, un grand nombre d'espèces attestant des climats plus chauds. D'autre part la répartition actuelle des espèces en Europe est en partie contrôlée climatiquement et peut donner également une indication sur l'environnement lors du dépôt.

Selon le premier critère, la présence de seulement 15 taxons de mollusques semblerait indiquer un environnement plutôt frais mais comme cette faune suggère fortement une dune ou une prairie côtière, le nombre des espèces peut être contrôlé plus par les conditions environnementales locales que par le climat régional. Les conditions rudes et sèches de la dune-prairie réduisent de manière caractéristique le nombre d'espèces présentes indépendamment du climat thermal et donc un petit nombre d'espèces ne peut être à lui seul l'indication d'un climat froid. De vraies faunes de climat froid trouvées dans le lœss de la région ont été rapportées par Rousseau \& Keen (1989) et ces faunes ne contiennent que 5 espèces ainsi qu'une liste d'espèces différentes incluant des espèces alpines et arctiques telle que Columella columella (von Martens).

Les répartitions actuelles de plusieurs des espèces de la pointe du Rozel sont présentées sur les cartes de Kerney \& Cameron (1979) (fig. 13). La très grande majorité de la faune a une répartition restreinte à la Scandinavie avec très peu de Truncatellina cylindrica et Helicella itala trouvées même dans le Sud de la Suède. De telles répartitions indiquent des étés pas plus froids qu'actuellement en Normandie bien que les hivers pouvaient être à la fois plus froids et continentaux. Si, comme le suggèrent les données stratigraphiques, l'horizon contenant les coquilles est d'âge Brörup, cela signifie que cet interstade était au moins aussi chaud qu'aujourd' hui durant les étés.

Par contre la liste de J.-J. Puisségur présente suffisamment d'espèces en commun avec la liste des espèces recensées dans la loupe de glissement pour en déduire que les mêmes conditions de prairie froide à tempérée y sont représentées.

\section{2 - LES VERTÉBRÉS (P. A.)}

\subsection{1 - Généralités}

Des vestiges osseux et dentaires de vertébrés ont été découverts au Rozel, essentiellement dans l'abri TR. 67, mais également au sein de plusieurs niveaux de la Grande Dune. La première étude de ces vestiges avait été confiée à différents spécialistes, M.-F. Bonifay pour les grands mammifères, J. Chaline pour la microfaune (rongeurs et insectivores), G. Desse et J. Granier pour les poissons et C. Mourer-Chauviré pour les oiseaux. Ces travaux ne concernaient que la collection Scuvée et une petite partie de la collection Roupin, l'inventeur du site. L'essentiel de leurs résultats a été publié dans le cadre de la monographie publiée en 1984 (Scuvée \& Verague, 1984). À l'occasion de la révision globale du gisement, D. Cliquet nous a remis l'intégralité du matériel (totalité des collections Scuvée \& Roupin) pour un réexamen en vue de clarifier en particulier le problème de l'âge attribué aux dépôts et d'établir la part de l'Homme quant aux accumulations osseuses mises au jour. 


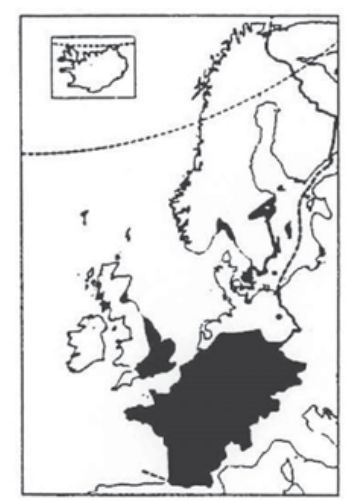

Truncatellina cylindrica

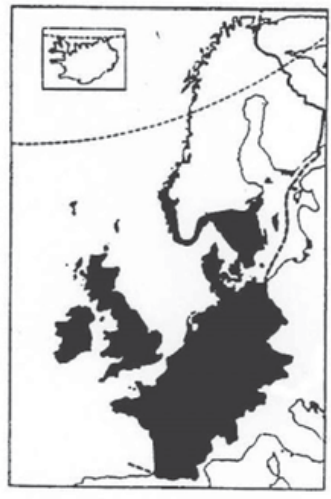

Discus rotundatus

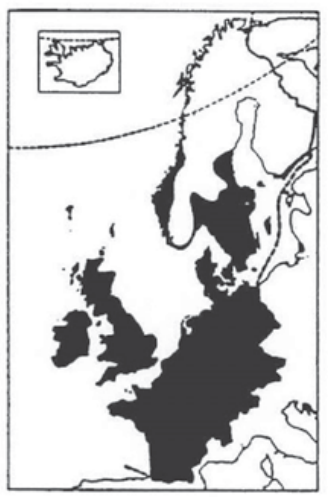

Vertigo pygmasa

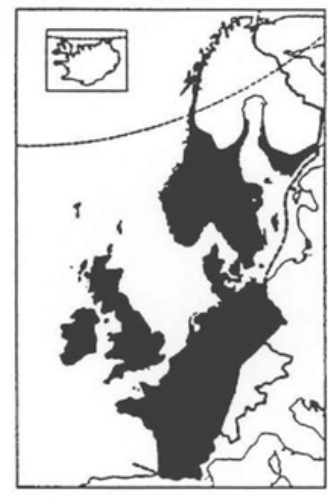

Clausilia bidentata

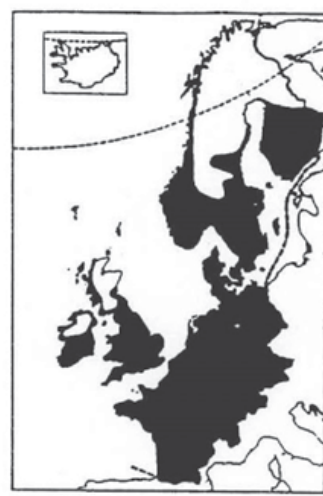

Vallonia costata

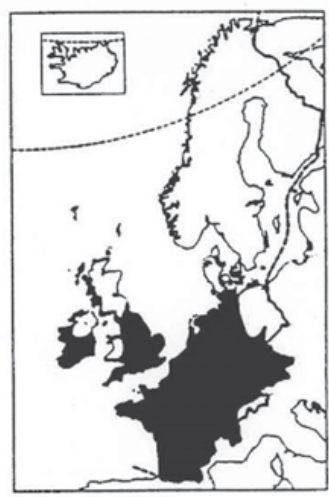

Helicalla itala

Fig. 13 : Cartes de distribution de Truncatellina cylindrica, de Vertigo pygmaea, de Vallonia costata, de Discus rotundatus, de Clausilia bidentata et de Helicella itala (d'après Kerney \& Cameron, 1979).

Fig. 13: Distribution maps for Truncatellina cylindrica, Vertigo pygmaea, Vallonia costata, Discus rotundatus, Clausilia bidentata and Helicella itala (after Kerney \& Cameron, 1979).

Un des premiers problèmes auquel nous avons été confrontés à été celui de la localisation précise des pièces, beaucoup d'entre elles ne portant souvent qu'un identifiant ne permettant pas forcément de distinguer l'abri TR. 67 de la Grande Dune, voire aucune indication. Malgré cela, l'état de conservation du matériel faunique permet au Rozel de séparer les vestiges provenant de TR. 67, globalement mal ou très mal conservés, de ceux des deux niveaux fossilifères de la Grande Dune où ils sont bien mieux préservés et parfois dans un excellent état. Cet état de fait entraîne ainsi une différence notable entre le décompte présenté en 1984 et les données du présent travail (tab. 5). Notre révision a porté par ailleurs uniquement sur les restes de grands mammifères, sans inclure les autres vertébrés dont la liste a été publiée en 1984 (Scuvée \& Verague, 1984). Ces petits vertébrés proviennent presque exclusivement de TR. 67 :

- Oiseau, du genre Pyrrhocorrax (Nombre de Restes = 7).

- Amphibien indéterminé (NR = 4).

- Rongeur indéterminé (NR = 3).

- Hérisson, Erinaceus europaeus $(\mathrm{NR}=1)$.

- Poisson, Labrus sp. (NR = 1).
Dans le niveau D6 de la Grande Dune, seuls les éléments du même membre antérieur d'un goéland argenté, Larus argentatus, ont été trouvés $(\mathrm{NR}=6)$.

5.2.2 - Les grands mammifères du Rozel : taxinomie, implications écologiques et biochronologiques

Le nombre total de restes de grands mammifères que nous avons analysés au Rozel est de 359, l'essentiel provenant à priori de TR. 67 (tab. 5). Nous avons pu déterminer anatomiquement et spécifiquement 133 pièces, soit $37 \%$ du matériel, les autres vestiges étant trop mal conservés pour permettre une diagnose de la nature de l'os et/ou du taxon. Il apparaît d'emblée une nette similitude entre les deux séries fauniques, celle de TR. 67 et de la Grande Dune, du point de vue de la représentation taxinomique. En effet, dans les deux cas c'est l'Aurochs (Bos primigenius) qui domine l'association, suivi du Cerf élaphe (Cervus elaphus), puis du Cheval (Equus sp.) (tab. 6). À ce triptyque de base, s'ajoute pour TR. 67 le Mégalocéros (Megaloceros giganteus), pour le niveau E2 de la Grande Dune le Morse (Odobenus cf. rosmarus). L'Aurochs est représenté en tout par au moins trois individus adultes, le Cerf par un adulte et deux jeunes, le Cheval par deux adultes, les deux autres 


\begin{tabular}{|c|c|c|c|}
\hline \multicolumn{3}{|l|}{ Le Rozel } & NR t \\
\hline \multirow[t]{4}{*}{ Grande Dune : } & \multirow[t]{2}{*}{ niveau D6 } & \multirow{2}{*}{$\begin{array}{l}\text { Scuvée } 1984 \\
\text { Auguste } 2006\end{array}$} & 11 \\
\hline & & & 7 \\
\hline & \multirow[t]{2}{*}{ niveau E2 } & \multirow{2}{*}{$\begin{array}{l}\text { Scuvée } 1984 \\
\text { Auguste } 2006\end{array}$} & 21 \\
\hline & & & 14 \\
\hline \multirow{2}{*}{\multicolumn{2}{|c|}{ Abri TR67 }} & \multirow{2}{*}{$\begin{array}{l}\text { Scuvée } 1984 \\
\text { Auguste } 2006\end{array}$} & $160+24$ coll Roupin \\
\hline & & & 29 \\
\hline Non attribués (TR67 ?) & & Auguste 2006 & 309 \\
\hline \multicolumn{3}{|c|}{ Total Auguste 2006 (coll Scuvée+Roupin) } & 359 \\
\hline
\end{tabular}

Tab. 5 : Le Rozel - Dénombrement des vestiges osseux de grands mammifères en fonction de la provenance stratigraphique (comparaison entre les données publiées par Scuvée \& Verague en 1984 et ce présent travail).

Tab. 5: Le Rozel - Counts of large mammal remains from all levels (comparison between Scuvée \& Verague 1984 and the present work).

\begin{tabular}{|l|r|c|}
\hline \multicolumn{1}{|c|}{ Le Rozel } & NRt & NMIc \\
\hline Aurochs (Bos primigenius) & 59 & 3 adultes \\
\hline Cheval (Equus sp.) & 22 & 2 adultes \\
\hline Cerf (Cervus elaphus) & 37 & 1 adulte +2 jeunes \\
\hline Morse (Odobenus rosmarus) & 1 & 1 adulte \\
\hline Megalocéros (Megaloceros giganteus) & 1 & 1 jeune \\
\hline petit Carnivore & 1 & 1 jeune \\
\hline Aurochs ou Cheval + indét & 238 & \\
\hline Total & $\mathbf{3 5 9}$ & 11 \\
\hline
\end{tabular}

Tab. 6 : Le Rozel - Dénombrement des ossements de grands mammifères (NRt : Nombre de Restes total ; NMIc : Nombre Minimum d’individus par combinaison).

Tab. 6: Le Rozel - Counts of large mammal remains (NRt: total number of elements; NMIc: minimal number of individuals)

taxons n'étant identifiés qu'au travers d'un seul individu, un adulte pour le Morse, un jeune pour le Mégalocéros (tab. 6). Une seule pièce rapportée à un petit Carnivore juvénile a également été reconnue, mais sans origine précise (probablement TR. 67).

Il faut signaler aussi la présence de huit pièces non fossilisées provenant probablement d'éboulis postpléistocènes et collectés par Y. Roupin (ont été identifiés : le Bœuf domestique, le Lapin, un Oiseau, une Chèvre ou un Mouton, un petit Mammifère).

L'Aurochs est représenté par plusieurs éléments dentaires et osseux post-crâniens, indiquant des individus de très forte taille et particulièrement robustes. La distinction morphologique avec le Bison a pu être effectuée sur les pièces les plus caractéristiques, comme les dents (fig. 14-a), les humérus (fig. 14-b), le radio-ulna, le métacarpien III-IV (fig. 14-c), le talus (fig. 14-d) (voir détails in Auguste, 1995). Dans tous les cas, la forme présente au Rozel s'apparente bien aux animaux connus durant le Pléistocène.

Des dimensions ont pu être par ailleurs relevées sur plusieurs pièces, permettant ainsi de confirmer d'une part l'affinité de l'Aurochs du Rozel avec ses homologues pléistocènes, d'autre part de mettre en exergue la très grande massivité des individus identifiés. C'est ainsi le cas pour les trois humérus, montrant des valeurs très proches de celles observées pour les populations de Biache-Saint-Vaast (Pas-de-Calais) et de Lunel-Viel (Hérault) (tab. 7). Le dimorphisme sexuel étant très bien marqué chez les grands Bovidés, la comparaison avec les mâles et les femelles de Lunel-Viel montrerait que nous avons affaire au Rozel à trois mâles. Le même constat est établi avec le radio-ulna qui indique aussi un mâle (tab. 8). Le capitato-trapézoïde mesuré montre également des valeurs très élevées (tab. 9). Les dimensions du talus vont aussi vers la caractérisation d'un individu mâle très massif (tab. 10).

Le Cerf élaphe est assez bien représenté au Rozel, avec des pièces qui indiquent comme pour l'Aurochs des individus de belle taille. Comme cela est souvent le cas au sein des accumulations osseuses ayant livré du Cerf au Paléolithique moyen, un bois de chute a également été retrouvé associé avec un animal abattu dans le niveau E2 de la Grande Dune, ce qui tendrait à indiquer la collecte de ce bois parallèlement à l'abattage d'un individu (fig. 15-a). La saisonnalité induite par ce bois de chute témoignerait d'une occupation à la fin de l'hiver ou au début du printemps. Au contraire, dans l'abri TR. 67, c'est un frontal portant la base d'un bois qui a été découvert, indiquant l'abattage d'au moins un mâle adulte (fig. 15-b). La saison correspondant à la mort de l'animal serait l'automne ou l'hiver. L'excellent état de conservation de quelques pièces découvertes au sein de la Grande Dune est particulièrement notable pour le Cerf, avec une hémimandibule portant toute la série dentaire (fig. 15-c ; tab. 11) et une scapula sub-entière (fig. 15-d).

Un Cheval a pu être identifié au Rozel, mais représenté par relativement peu de vestiges. La taxinomie et la caractérisation des différentes formes caballines du Pléistocène posent encore à l'heure actuelle de nombreuses questions, compte-tenu de la plasticité 


\begin{tabular}{|c|c|c|c|c|c|c|}
\hline Aurochs, humérus & DT troc méd & ht troc méd & DT art & DT troc lat & ht gorge & ht troc lat \\
\hline Le Rozel : $\quad ?$ & 75,0 & 62,1 & 109,0 & 29,3 & 38,3 & \\
\hline G. Dune, niv E2, no E 167 & 72,8 & 58,4 & 104,5 & 31,4 & 46,5 & 38,7 \\
\hline Dune, niv D6, nº R9-34/G 34-I & 84,0 & 64,5 & & & & \\
\hline Biache (1) & $\begin{array}{c}77,5 \\
(63 ; 64,0-86,2)\end{array}$ & $\begin{array}{c}64,3 \\
(67 ; 53,5-72,2) \\
\end{array}$ & $\begin{array}{c}109,7 \\
(62 ; 92,0-122,1) \\
\end{array}$ & $\begin{array}{c}31,4 \\
(71 ; 23,4-37,5)\end{array}$ & $\begin{array}{c}49,74 \\
(53 ; 41,5-59,0)\end{array}$ & $\begin{array}{c}48,34 \\
(52 ; 39,4-57,3)\end{array}$ \\
\hline \multirow[t]{2}{*}{ Lunel-Viel (2) : } & $\begin{array}{c}76,98 \\
(9 ; 72,7-80,8)\end{array}$ & \begin{tabular}{c|}
68,58 \\
$(9 ; 62,8-74,9)$ \\
\end{tabular} & $\begin{array}{c}110,21 \\
(9 ; 103,0-116,1)\end{array}$ & $\begin{array}{c}33,16 \\
(9 ; 29,0-36,0)\end{array}$ & $\begin{array}{c}58,97 \\
(7 ; 55,8-62,4)\end{array}$ & $\begin{array}{c}46,86 \\
(6 ; 42,0-51,0)\end{array}$ \\
\hline & $\begin{array}{c}64,9 \\
(25 ; 62,1-70,5)\end{array}$ & $\begin{array}{c}58,44 \\
(26 ; 54,9-65,9)\end{array}$ & $\begin{array}{c}92,49 \\
(26 ; 85,5-98,2)\end{array}$ & $\begin{array}{c}28,11 \\
(26 ; 22,8-32,0)\end{array}$ & $\begin{array}{c}51,56 \\
(26 ; 48,7-54,8)\end{array}$ & $\begin{array}{c}40,16 \\
(21 ; 35,1-44,8)\end{array}$ \\
\hline
\end{tabular}

Tab. 7 : Comparaison des dimensions des humérus des Aurochs du Rozel avec celles des Aurochs de Biache-Saint-Vaast et de Lunel-Viel [(1) : Auguste, 1995 ; (2) : Brugal, 1983 ; la première valeur indique la moyenne, entre parenthèse suivent le nombre de spécimens et l'intervalle de variation observé] (DT troc méd : diamètre transverse de la trochlée médiale ; ht troc méd : hauteur de la trochlée médiale ; DT art : diamètre transverse articulaire ; DT troc lat : diamètre transverse de la trochlée latérale ; ht gorge : hauteur de la gorge trochléaire ; ht troc lat : hauteur de la trochlée latérale).

Tab. 7: Comparative measurements of wild ox humerus from Le Rozel with other populations from Biache-Saint-Vaast and Lunel-Viel [(1): Auguste, 1995; (2): Brugal, 1983; the first number gives the average, the number of specimens and the minimum and maximum values are in brackets] (DT troc méd: breadth of medial trochlea; ht troc méd: height of medial trochlea; DT art: breath of the articulation; DT troc lat: breadth of lateral trochlea; ht gorge: height of trochleare groove; ht troc lat: height of lateral trochlea).

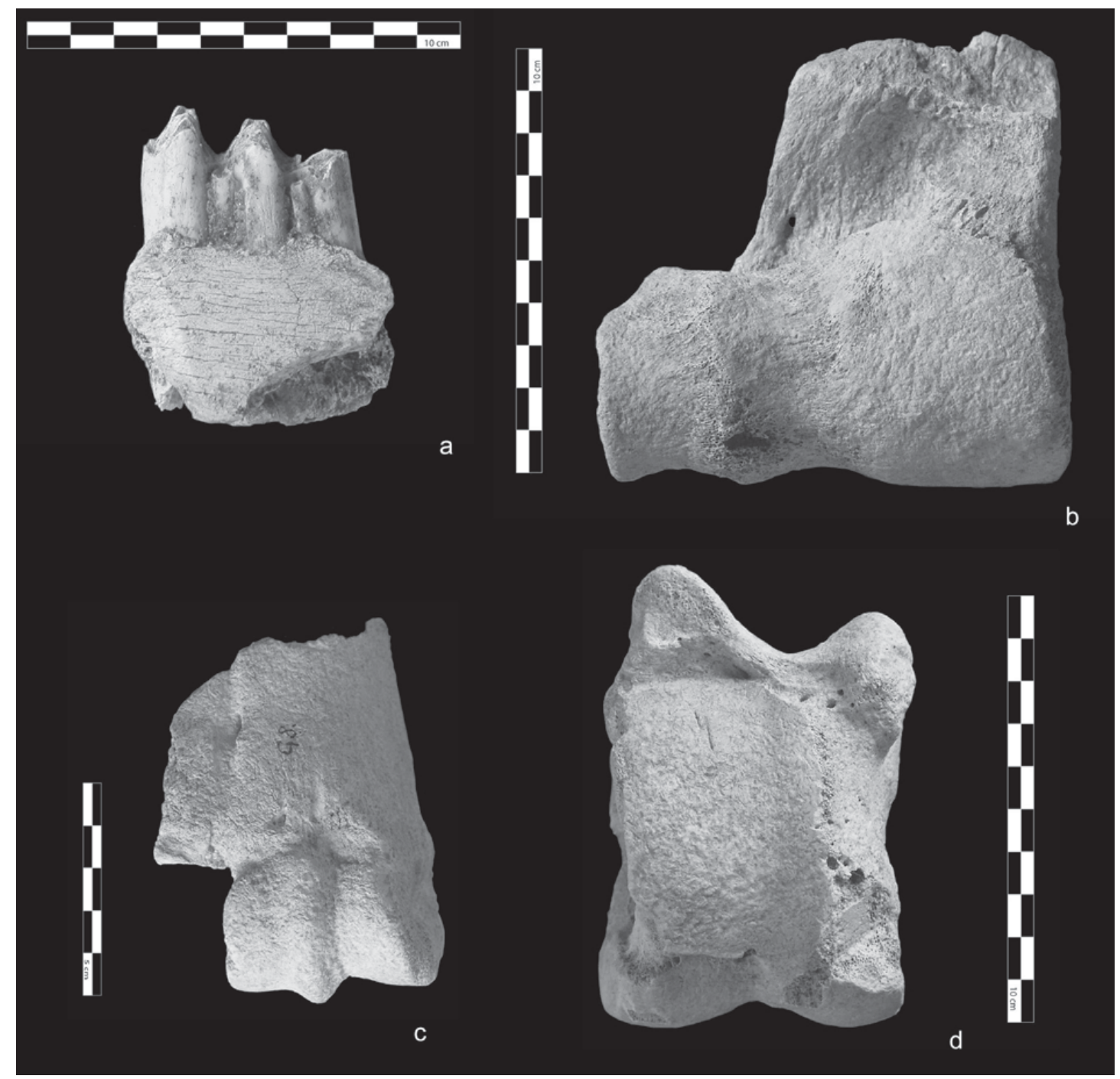

Fig. 14 : Le Rozel - Aurochs : (a) hémimandibule gauche portant la troisième molaire $\left(\mathrm{n}^{0}\right.$ E3-1), vue vestibulaire, (b) humérus droit (Grande Dune, niveau E2, $\mathbf{n}^{\circ}$ E 167), vue dorsale, (c) métacarpien III-IV (Grande Dune, niveau D6, $\mathbf{n}^{\circ}$ G8), vue dorsale et (d) talus gauche (Grande Dune, niveau D6, $\mathbf{n}^{\circ}$ G35/I-R935), vue plantaire.

Fig. 14: Le Rozel - Wild ox: (a) left mandible with third molar ( $n^{\circ}$ E3-1), vestibular view, (b) right humerus (Grande Dune, level E2, $n^{\circ}$ E 167), dorsal view, (c) metacarpal III-IV (Grande Dune, level D6, $n^{\circ}$ G8), dorsal view and (d) left talus (Grande Dune, level D6, $n^{\circ}$ G35/I-R935), plantar view. 


\begin{tabular}{|l|c|l|c|}
\hline Aurochs, radio-ulna & Le Rozel $\left(\right.$ TR 67, $\mathbf{n}^{\mathbf{0}} \mathbf{0}$ & \multicolumn{1}{|c|}{ Biache $(\mathbf{1})$} & Lunel-Viel, mâles (2) \\
\hline DT max dist & 124,2 & $\mathbf{1 0 0 , 9 0}(25 ; 89,8-133,5)$ & $\mathbf{1 0 8 , 9 0}(7 ; 102,5-118,3)$ \\
\hline DDP max dist & 79,6 & $\mathbf{6 3 , 8}(24 ; 57,5-79,0)$ & $\mathbf{6 8 , 7 0}(7 ; 61,0-75,0)$ \\
\hline DT art dist & 108,8 & $\mathbf{9 2 , 7 2}(28 ; 80,7-115,1)$ & $\mathbf{1 0 2 , 3 4}(7 ; 94,6-111,5)$ \\
\hline DDP art dist & 54,3 & $\mathbf{4 8 , 0}(39 ; 36,8-57,0)$ & $\mathbf{5 2 , 9 0}(7 ; 49,3-56,5)$ \\
\hline DT art pyramidal & 32,9 & $\mathbf{3 0 , 0 1}(60 ; 22,5-37,2)$ & $\mathbf{3 1 , 0 5}(6 ; 25,9-34,0)$ \\
\hline DT art semi-lunaire & 42,4 & $\mathbf{3 8 , 4 0}(46 ; 33,1-44,2)$ & $\mathbf{4 1 , 5 2}(7 ; 40,0-43,6)$ \\
\hline DT art scaphoVde & 47,3 & $\mathbf{3 6 , 9 0}(86 ; 29,3-46,1)$ & $\mathbf{3 1 , 0 1}(7 ; 36,0-43,7)$ \\
\hline DT art médial & 63,7 & $\mathbf{5 4 , 6 0}(57 ; 45,4-66,8)$ & $\mathbf{5 7 , 6 5}(7 ; 51,5-62,4)$ \\
\hline DT art latéral & 44,6 & $\mathbf{4 2 , 7 0}(34 ; 35,0-52,4)$ & $\mathbf{4 3 , 1 8}(6 ; 38,5-50,6)$ \\
\hline
\end{tabular}

Tab. 8 : Comparaison des dimensions du radio-ulna de l'Aurochs du Rozel avec celles des Aurochs de Biache-Saint-Vaast et de Lunel-Viel [(1) : Auguste, 1995 ; (2) : Brugal, 1983] (DT max dist : diamètre transverse maximum distal ; DDP max dist : diamètre dorso-palmaire maximum distal ; DT art dist : diamètre transverse articulaire distal ; DDP art dist : diamètre dorso-palmaire articulaire distal ; DT art pyramidal : diamètre transverse de l'articulation pour le pyramidal ; DT art semi-lunaire : diamètre transverse de l'articulation pour le semi-lunaire ; DT art scaphoïde : diamètre transverse de l'articulation pour le scaphö̈de ; DT art médial : diamètre transverse articulaire médial ; DT art latéral : diamètre transverse articulaire latéral).

Tab. 8: Comparative measurements of wild ox radio-ulna from Le Rozel with others populations from Biache-Saint-Vaast and Lunel-Viel [(1): Auguste, 1995; (2): Brugal, 1983] (DT max dist: distal maximum breadth; DDP max dist: distal dorso-palmar maximum breadth; DT art dist: articular distal breadth; DDP art dist: dorso-palmar articular distal breadth; DT art pyramidal: pyramidal articulation breadth; DT art semi-lunaire: semi-lunaire articulation breadth; DT art scaphö̈de: scaphö̈de articulation breadth; DT art medial: medial articulation breadth; DT art latéral: lateral articular breadth).

\begin{tabular}{|l|c|c|}
\hline Aurochs, capitato-trapézoïde & Le Rozel (TR 67, $\mathbf{n}^{\mathbf{0}}$ X-01) & Biache (1) \\
\hline Ht bord dorsal & 24,8 & $\mathbf{2 1 , 1 9}(18 ; 18,4-24,8)$ \\
\hline ht bord palm & 27,8 & $\mathbf{2 2 , 7 2}(18 ; 17,1-27,0)$ \\
\hline DT & 52,3 & $\mathbf{5 1 , 3 2}(18 ; 41,0-63,5)$ \\
\hline DDP max & 51,5 & $\mathbf{4 9 , 7 6}(17 ; 40,5-57,2)$ \\
\hline DDP min & 47,9 & $\mathbf{4 6 , 8 5}(17 ; 40,4-54,1)$ \\
\hline DDP max/DT & 91,59 & $\mathbf{9 1 , 4 0}(17 ; 82,99-98,54)$ \\
\hline
\end{tabular}

Tab. 9 : Comparaison des dimensions du capitato-trapézoïde de l'aurochs du Rozel avec celles des aurochs de Biache-Saint-Vaast [(1) : Auguste, 1995] (ht bord dorsal : hauteur du bord dorsal ; ht bord palmaire : hauteur du bord palmaire ; DT : diamètre transverse ; DDP max : diamètre dorso-palmaire maximum ; DDP min : diamètre dorso-palmaire minimum).

Tab. 9: Comparative measurements of wild ox capitato-trapézö̈de from Le Rozel with other populations from Biache-Saint-Vaast and Lunel-Viel [(1): Auguste, 1995] (ht bord dorsal: dorsal side height; ht bord palmaire: palmar side height; DT: breath; DDP max: maximum dorso-palmar breadth; DDP min: minimum dorso-palmar breadth).

\begin{tabular}{|c|c|c|c|c|}
\hline \multirow[t]{2}{*}{ Aurochs, talus } & \multirow{2}{*}{$\begin{array}{c}\text { Le Rozel (G. Dune, } \\
\text { niv D6, } \\
\text { no G35/I-R935) }\end{array}$} & \multirow[t]{2}{*}{ Biache (1) } & \multicolumn{2}{|c|}{ Lunel-Viel (2) } \\
\hline & & & mâles & femelles \\
\hline L lat & 101,8 & $\mathbf{9 6 , 0}(28 ; 85,7-104,3)$ & $\mathbf{9 6 , 7 9}(14 ; 91,1-100,8)$ & $\mathbf{8 7 , 4 9}(66 ; 81,7-93,3)$ \\
\hline L méd & 92,8 & $\mathbf{8 6 , 7 9}(32 ; 79,5-98,9)$ & $\mathbf{8 6 , 7 4}(12 ; 84,1-93,8)$ & $\mathbf{7 8 , 9 2}(66 ; 74,2-87,9)$ \\
\hline DDP lat & 58,9 & $\mathbf{5 4 , 2 4}(28 ; 48,7-58,8)$ & $\mathbf{5 4 , 4 5}(14 ; 51,2-57,2)$ & $\mathbf{4 8 , 9 3}(66 ; 45,0-53,1)$ \\
\hline DDP méd & 60,7 & $\mathbf{5 5 , 0 5}(30 ; 49,8-59,9)$ & $\mathbf{5 7 , 1 2}(12 ; 52,2-63,1)$ & $\mathbf{5 0 , 1 3}(66 ; 46,3-54,5)$ \\
\hline DT prox & 65,5 & $\mathbf{6 3 , 3 9}(26 ; 56,1-71,5)$ & $\mathbf{6 3 , 9 5}(12 ; 58,9-72,1)$ & $\mathbf{5 6 , 6 9}(65 ; 50,2-60,1)$ \\
\hline DT médian & 63,0 & $\mathbf{5 9 , 3 7}(30 ; 51,5-66,2)$ & $\mathbf{6 1 , 3 9}(14 ; 58,5-64,8)$ & $\mathbf{5 3 , 6 4}(67 ; 48,1-58,3)$ \\
\hline DT dist & 68,3 & $\mathbf{6 5 , 3 3}(34 ; 55,0-74,7)$ & $\mathbf{6 7 , 9 5}(13 ; 65,2-75,2)$ & $\mathbf{5 7 , 8 4}(65 ; 50,5-63,6)$ \\
\hline L art calcanéus & 20,3 & $\mathbf{1 7 , 1 6}(32 ; 14,0-20,6)$ & $\mathbf{1 6 , 1 4}(13 ; 13,8-19,8)$ & $\mathbf{1 4 , 4 4}(64 ; 11,8-18,2)$ \\
\hline DDP art calcanéus & 40,3 & $\mathbf{3 0 , 5 5}(20 ; 23,7-36,3)$ & $\mathbf{3 0 , 8 8}(12 ; 27,1-36,1)$ & $\mathbf{2 6 , 3 2}(52 ; 21,3-31,8)$ \\
\hline DT méd troc dist & 31,8 & 32,09 $(34 ; 27,3-37,6)$ & $\mathbf{3 2 , 9 2}(14 ; 27,5-37,6)$ & $\mathbf{2 8 , 0 3}(66 ; 23,0-31,3)$ \\
\hline DT lat troc dist & 34,7 & $\mathbf{3 2 , 5 9}(34 ; 27,9-37,4)$ & $\mathbf{3 3 , 8 5}(14 ; 31,1-36,5)$ & $\mathbf{2 9 , 2 6}(65 ; 25,3-32,8)$ \\
\hline DDP lat/L lat & 57,86 & $\mathbf{5 6 , 5 6}(27 ; 52,76-59,72)$ & \multicolumn{2}{|c|}{$\mathbf{5 5 , 9 8}(79 ; 52,68-59,17)$} \\
\hline $\mathrm{L}$ art calca/DDP art calca & $\mathbf{5 0 , 3 7}$ & $\mathbf{5 7 , 0 1}(20 ; 46,56-67,62)$ & \multicolumn{2}{|c|}{$\mathbf{5 5 , 1 4}(66 ; 41,51-67,96)$} \\
\hline DT méd troc/DT lat troc & 91,64 & $\mathbf{9 8 , 7 3}(34 ; 85,40-115,0)$ & \multicolumn{2}{|c|}{$\mathbf{9 6 , 6 5}(77 ; 83,91-115,79)$} \\
\hline
\end{tabular}

Tab. 10 : Comparaison des dimensions du talus de l'aurochs du Rozel avec celles des Aurochs de Biache-Saint-Vaast et de Lunel-Viel [(1) : Auguste, 1995 ; (2) : Brugal, 1983 ] (L lat : longueur latérale ; L méd : longueur médiale ; DDP lat : diamètre dorso-plantaire latéral ; DDP méd : diamètre dorso-plantaire médial ; DT prox : diamètre transverse proximal ; DT médian : diamètre transverse médian ; DT dist : diamètre transverse distal ; $\mathrm{L}$ art calcanéus : longueur de l'articulation pour le calcanéus ; DDP art calcanéus : diamètre dorso-plantaire de l'articulation pour le calcanéus ; DT méd troc dist : diamètre transverse médial de la trochlée distale ; DT lat troc dist : diamètre transverse latéral de la trochlée distale).

Tab. 10: Comparative measurements of wild ox talus from Le Rozel with other populations from Biache-Saint-Vaast and Lunel-Viel [(1): Auguste, 1995; (2): Brugal, 19837 (L lat: lateral length; L méd: medial length; DDP lat: lateral dorso-plantar breadth; DDP méd: medial dorso-plantar breadth; DT prox: proximal breadth; DT median: median breadth; DT dist: distal breadth; L art calcanéus: calcaneus articulation length; DDP art calcanéus: dorsoplantar calcaneus articulation breadth; DT méd troc dist: distal trochlea medial breadth; DT lat troc dist : distal trochlea lateral breadth). 


\begin{tabular}{|l|c|c|}
\hline $\begin{array}{l}\text { Cerf, dents inférieures } \\
\text { Le Rozel (Grande Dune } \\
\text { niveau D6, } \mathbf{n}^{\mathbf{0}} \text { R9-1/G1-I) }\end{array}$ & L & $\mathbf{1}$ \\
\hline P2 inf & & 7,8 \\
\hline P3 inf & 17,0 & 11,4 \\
\hline P4 inf & 19,1 & 13,3 \\
\hline M1 inf & 22,3 & 14,7 \\
\hline M2 inf & 25,5 & 16,2 \\
\hline M3 inf & 36,1 & 15,5 \\
\hline
\end{tabular}

Tab. 11 : Le Rozel - Dimensions des dents inférieures du Cerf (L : longueur ; 1 : largeur).

Tab. 11: Le Rozel - Red deer lower cheek teeth measurements (L: length ; l: breadth).

\begin{tabular}{|l|l|l|l|l|}
\hline Cheval, dents supérieures & & & & \\
\hline $\begin{array}{l}\text { Le Rozel (Grande Dune, } \\
\text { niveau E2, n }{ }^{\circ} \text { E5, E6, E7, E8) }\end{array}$ & L oc & l oc & LP oc & IP oc \\
\hline P3 sup & 31,5 & 28,4 & 13,0 & 41,27 \\
\hline P4 sup & 31,7 & 28,2 & 14,5 & 45,74 \\
\hline M1 sup & 28,4 & 26,7 & 12,5 & 44,01 \\
\hline M2 sup & 28,7 & 27,7 & 12,9 & 44,95 \\
\hline
\end{tabular}

Tab. 12 : Le Rozel - Dimensions des dents supérieures du cheval ( $L$ oc : longueur occlusale ; l oc : largeur occlusale ; LP oc : longueur occlusale du protocône ; IP oc : indice protoconique occlusal).

Tab. 12: Le Rozel-Horse upper cheek teeth measurements (Loc: occlusal length; l oc: occlusal breadth; LP oc: occlusal protocone length; IP oc: occlusal protoconic index).

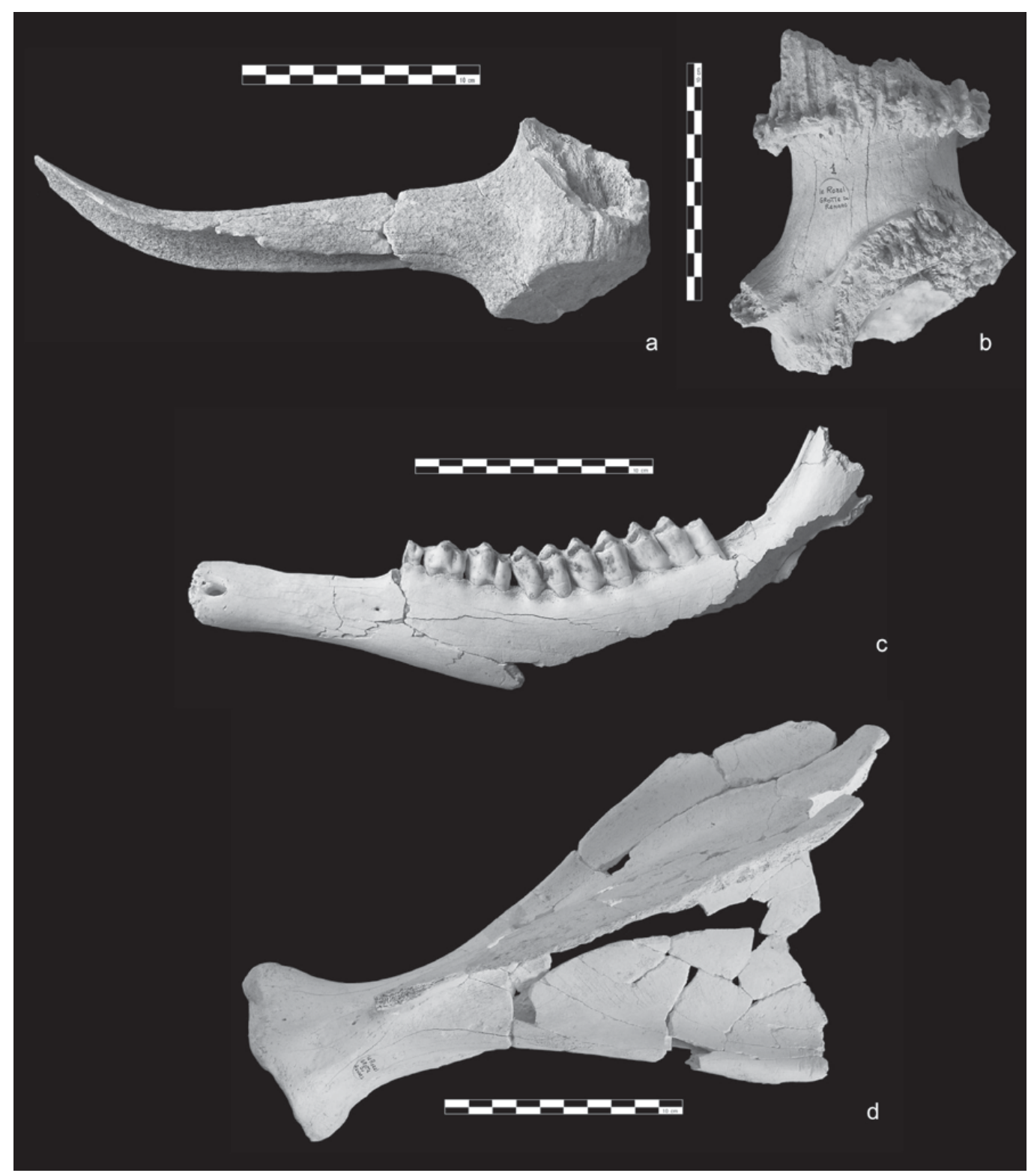

Fig. 15 : Le Rozel - Cerf : (a) bois de chute (Grande Dune, niveau E2, $\mathbf{n}^{0}$ E168), (b) crâne avec départ du bois gauche (TR 67, $\mathrm{n}^{0}$ 1), vue médiale, (c) hémimandibule gauche portant la série dentaire P2 à M3 (Grande Dune, niveau D6, $\mathbf{n}^{0}$ R9-1/G1-I), vue vestibulaire et (d) scapula gauche (sans $\mathbf{n}^{0}$ ), vue dorsale.

Fig. 15 : Le Rozel-Red deer : (a) antler (Grande Dune, level E2, $n^{\circ}$ E168), (b) skull with left antler base (TR 67, $\left.n^{\circ} 1\right)$, medial view., (c) left mandible with P2 to M3 (Grande Dune, level D6, $n^{o}$ R9-1/G1-I), vestibular view and (d) left scapula (without $\left.n^{\circ}\right)$, dorsal view. 


\begin{tabular}{|c|c|c|c|c|c|c|c|}
\hline $\begin{array}{l}\text { Cheval, } \\
\text { dents supérieures }\end{array}$ & $\begin{array}{c}\text { Le Rozel (Grande } \\
\text { Dune, } \\
\text { niveau E2, } \\
n^{\circ} \text { E5, E6, E7, E8) }\end{array}$ & $\begin{array}{c}\text { Taubach (1) } \\
\text { E. taubachensis }\end{array}$ & $\begin{array}{l}\text { Bettencourt (2) } \\
\text { Equus sp. }\end{array}$ & \begin{tabular}{|c|} 
Combe-Grenal \\
$22-25(1)$ \\
Equus sp.
\end{tabular} & $\begin{array}{l}\text { Beauvais } \\
\text { Equus sp. }\end{array}$ & $\begin{array}{c}\text { Remagen (1) } \\
\text { E. germanicus }\end{array}$ & $\begin{array}{c}\text { Solutré } \\
\text { (Aurig-Périg) (3) } \\
\text { E. gallicus }\end{array}$ \\
\hline P Long & 31,6 & 30,2 & 29,4 & 30,8 & 31,1 & 28,0 & 28,6 \\
\hline M Long & 28,55 & 27,4 & 25,6 & 27,6 & 28,1 & 25,8 & 26,0 \\
\hline$L$ prot $P$ & 13,75 & 14,3 & 13,0 & 14,4 & 16,0 & 10,8 & 13,5 \\
\hline$L \operatorname{prot} M$ & 12,7 & 14,7 & 12,1 & 14,1 & 14,5 & 13,5 & 14,4 \\
\hline L P3M2 & 30,07 & 28,8 & 27,13 & 29,2 & 29,6 & 26,9 & 27,3 \\
\hline L prot P3M2 & 13,23 & 14,5 & 12,5 & 14,3 & 15,3 & 12,1 & 13,95 \\
\hline IP oc M & 44,48 & 53,6 & 47,45 & 51,1 & 51,72 & 52,3 & 55,69 \\
\hline IP oc P & 43,51 & 47,4 & 44,08 & 46,8 & 51,45 & 38,6 & 47,42 \\
\hline IP oc M/IP oc P & 102,23 & 109,6 & 108,42 & 118,4 & 100,52 & 126,3 & 117,44 \\
\hline
\end{tabular}

Tab. 13 : Comparaison des dimensions des dents supérieures du cheval du Rozel avec celles de plusieurs chevaux du Pléistocène supérieur [(1) : Eisenmann, 1991 ; (2) : Auguste, 2002 ; (3) : Guadelli, 1991] (P Long : longueur moyenne des prémolaires ; M Long : longueur moyenne des molaires ; L prot $\mathrm{P}$ : longueur moyenne des protocônes des prémolaires ; L prot M : longueur moyenne des protocônes des molaires ; L P3M2 : longueur moyenne des prémolaires et molaires; L prot P3M2 : longueur moyenne des protocônes des prémolaires et molaires ; IP oc M : indice protoconique moyen occlusal des molaires ; IP oc P : indice protoconique moyen occlusal des prémolaires).

Tab. 13 : Comparative measurements of horse upper cheek teeth from Le Rozel with other Upper Pleistocene populations [(1) : Eisenmann, 1991 ; (2) : Auguste, $2002 ;(3)$ : Guadelli, 1991] (P Long : premolars average length; $M$ Long : molars average length; L prot $P$ : premolars protocone average length; L prot $M:$ molars protocone average length; L P3M2: premolars and molars average length; L prot P3M 2 : premolars and molars protocone average length ; IP oc $M$ : molars protoconic occlusal average index; IP oc P : premolars protoconic occlusal average index).

morphologique observée chez ces animaux et la présence de plusieurs formes distinctes suivant les contextes écologiques et les périodes considérées. C'est en particulier le cas pour la première phase du Pléistocène supérieur où il est très difficile d'identifier spécifiquement les différentes populations étudiées. Le problème s'est ainsi posé au Rozel, mais nous avons tenté malgré tout de définir au mieux le Cheval présent.

Une série dentaire supérieure composée des troisième et quatrième prémolaires ainsi que des première et deuxième molaires nous a servi de base de travail morphométrique (tab. 12). Nous avons pour cela utilisé les différents critères développés par V. Eisenmann (1991), basés sur les longueurs des différentes dents supérieures et les longueurs des protocônes de ces dents. Nous avons ensuite comparé les valeurs obtenues pour le Cheval du Rozel avec celles de Chevaux dont le contexte chronostratigraphique et écologique est bien connu et ce, pour une période couvrant presque l'intégralité du Pléistocène supérieur (tab. 13). Les longueurs dentaires de ce Cheval indiquent ainsi des dents assez grosses, comparables à celles relevées chez plusieurs Chevaux du début du Pléistocène supérieur, comme à Taubach (contemporanéité avec le stade isotopique 5.5 ; Eisenmann, 1991) et de la première moitié de cette même période comme à Beauvais (stade isotopique 4 ; Auguste, données inédites) ou Combe-Grenal niveaux 22-25 (stade isotopique 4 ; Eisenmann, 1991). Par contre, elles apparaissent bien plus massives que leurs homologues chez les Chevaux de la seconde partie du Pléistocène supérieur, comme à Remagen pour l'Equus germanicus type (stade isotopique 3; Eisenmann, 1991) ou dans les niveaux anciens de Solutré avec l'E. gallicus type (stade isotopique 2; Guadelli, 1991). Ce constat rejoint celui effectué par V. Eisenmann (citée in lit. par F. Poplin, courrier à F. Scuvée du 03-12-1992). La longueur des protocônes montre des valeurs proches de celles des Chevaux de Bettencourt (stade isotopique 5.1; Auguste, 2002) et de l'E. gallicus de Solutré. Les indices protoconiques sont par contre faibles, assez similaires à ceux du Cheval de Bettencourt, bien en deçà de ceux des Chevaux de la seconde partie du Pléistocène supérieur. Le rapport des indices protoconiques molaires sur prémolaires confirme l'affinité avec les chevaux les plus anciens du Pléistocène supérieur et exclut les formes contemporaines des stades isotopiques 3 et 2 .

D'après ces données, nous sommes amenés à rapprocher le Cheval du Rozel des formes identifiées durant le début du Pléistocène supérieur, plus spécifiquement des Chevaux aux indices protoconiques peu élevés comme à Bettencourt.

Les dents inférieures montrent également une certaine robustesse, excluant à leur tour les Chevaux du Pléistocène supérieur récent (fig. 16-a et b ; tab. 14).

\begin{tabular}{|c|c|c|c|c|c|}
\hline \multirow{2}{*}{$\begin{array}{l}\text { Cheval, dents inférieures } \\
\text { Le Rozel (E2-3) }\end{array}$} & \multirow[b]{2}{*}{ P2 inf } & \multirow[b]{2}{*}{$\begin{array}{l}\text { P3 } \\
\text { inf }\end{array}$} & \multirow[b]{2}{*}{$\begin{array}{l}\text { P4 } \\
\text { inf }\end{array}$} & \multirow[b]{2}{*}{$\begin{array}{l}\text { M1 } \\
\text { inf }\end{array}$} & \multirow[b]{2}{*}{$\begin{array}{l}\text { M2 } \\
\text { inf }\end{array}$} \\
\hline & & & & & \\
\hline L oc & 37,3 & 32,0 & 31,8 & 28,5 & 29,1 \\
\hline l oc & 15,1 & 17,1 & 17,2 & 15,2 & 15,2 \\
\hline L prf & 9,7 & 10,5 & 9,7 & 9,5 & 9,5 \\
\hline L pof & 17,3 & 15,7 & 14,6 & 10,9 & 10,5 \\
\hline $\mathbf{L} \mathbf{d b}$ & 17,0 & 18,5 & 17,3 & 15,1 & 14,5 \\
\hline L P2-M2 & 157,2 & & & & \\
\hline $\mathbf{L} \mathbf{P}$ & 99,5 & & & & \\
\hline L M1-M2 & 56,9 & & & & \\
\hline
\end{tabular}

Tab. 14 : Le Rozel - Dimensions des dents inférieures du cheval (L oc : longueur occlusale ; l oc : largeur occlusale; L prf : longeur du préflexide ; $\mathrm{L}$ pof : longueur du postflexide ; $\mathrm{L}$ db : longueur de la double-boucle ; L P2-M2 : longueur de la série dentaire ; L P : longueur de la série prémolaire ; L M1-M2 : longueur de la série molaire conservée).

Tab. 14: Le Rozel - Horse lower cheek teeth measurements (L oc: occlusal length; loc: occlusal breadth; L prf: pre-flexide length; L pof: postflexide length; $L d b$ : double knot length; L P2-M2: dental range length; $L P$ : premolar range length; LM1-M2 : preserved molar range length). 


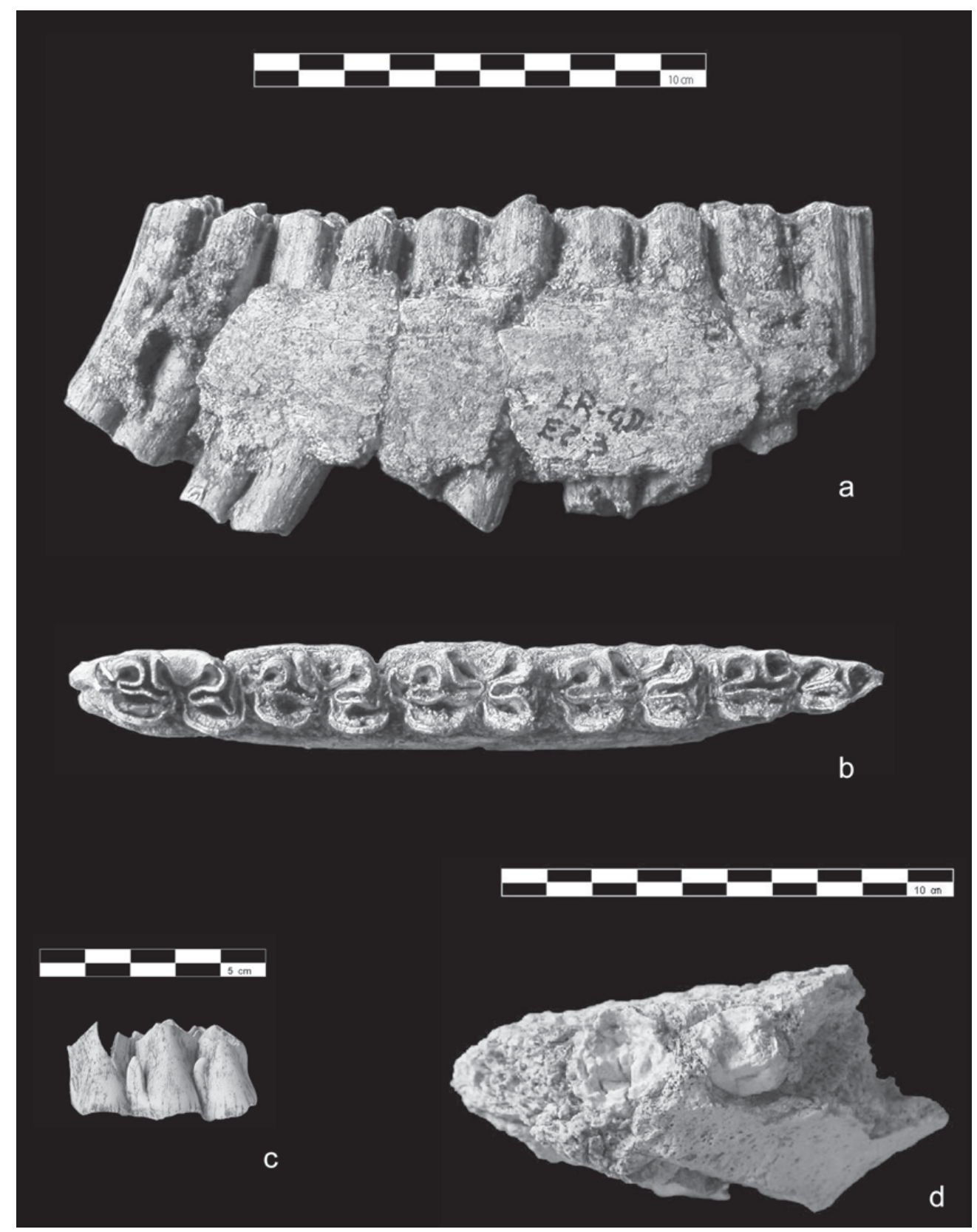

Fig. 16 : Le Rozel - Cheval : hémimandibule droite portant la série dentaire P2 à M2 (nº E2-3) ; (a) vue linguale ; (b) vue occlusale ; Mégalocéros : (c) bourgeon troisième molaire inférieure gauche (TR 67, $n^{\circ} \mathrm{H1} \mathrm{048),} \mathrm{vue} \mathrm{vestibulaire),} \mathrm{et} \mathrm{Morse} \mathrm{:} \mathrm{(d)} \mathrm{hémimandibule} \mathrm{gauche} \mathrm{(Grande} \mathrm{Dune,} \mathrm{ni-}$ veau E2), vue occlusale.

Fig. 16: Le Rozel - Horse: right mandible with P2 to M2 ( $n^{o}$ E2-3); (a) lingual view; (b) occlusal view; Megaloceros: (c) left third inferior molar (TR 67, $n^{\circ}$ H1 048), vestibular view, and Walrus: (d) left mandible (Grande Dune, niveau E2), occlusal view.

Le Mégalocéros n'a été identifié qu'à partir d'un seul vestige, un bourgeon dentaire de troisième molaire inférieure (fig. 16-c).

La pièce la plus «extraordinaire » découverte au Rozel n'a été reconnue que postérieurement à la publication de la monographie de 1984 et est donc restée inédite à ce jour. Il s'agit d'un fragment de symphyse mandibulaire portant les racines des prémolaires antérieures d'un Morse (fig. 16-d). Identifiée pour la première fois par L. Ginsburg (in lit., lettre de L. Ginsburg à F. Scuvée du 05-09-1990), cette détermination a été confirmée par F. Poplin qui a présenté ce vestige dans le cadre d'un manuscrit demeuré inédit (« Premières découvertes de morse préhistorique en France et autres histoires de bêtes à cornes ", Poplin, 1992). L'importance de cet ossement est liée à l'extrême rareté de la découverte de restes de Pinnipèdes fossiles de manière générale et de Morse en particulier (de Sonneville-Bordes \& Laurent, 1983). À notre connaissance seules les deux pièces mentionnées par F. Poplin ont été décrites en France pour ce dernier animal (un crâne découvert en Gironde et la présente mandibule du 
Rozel). Des fossiles de Morse pléistocène sont néanmoins connus aux Pays-Bas et dans le nord de la Belgique ; ils ont été rapportés à une espèce distincte du Morse actuel et dénommée Odobenus antwerpiensis (Bosscha-Erdbrink \& Van Bree, 1990). Il est malheureusement impossible à partir du vestige du Rozel de déterminer précisément l'espèce présente, nous attribuons donc cet os à $O$. c f. rosmarus dans l'état actuel des données.

Bien que les associations fauniques de l'abri TR. 67 et de la Grande Dune soient très restreintes et représentées par peu de restes, il est toutefois possible d'apporter des éléments au débat concernant l'âge et le contexte climatique des occupations humaines au Rozel.

D'un point de vue biochronologique, seul le Cheval peut apporter des arguments permettant de le rapprocher des formes du début du Pléistocène supérieur, en excluant assez nettement les Chevaux plus évolués de la fin de cette période. C'est peut être avec le Cheval identifié à Bettencourt que l'analogie semble être la plus probable dans l'état actuel des données. Laissé en nomenclature ouverte d'Equus sp., ce Cheval pourrait représenter une forme de transition entre E. taubachensis et E. germanicus (Auguste, 2002). Il se retrouve en contexte tempéré, associé à l'Aurochs, en contemporanéité avec le stade isotopique 5.1. La prudence doit bien entendu être de rigueur, n'ayant dans les deux cas affaire qu'à des individus et non à des populations vraiment représentatives. Il est néanmoins possible de conforter une datation à la fois chronostratigraphique et géochronologique des occupations du Rozel au cours d'une période correspondant au début du Pléistocène supérieur telle qu'elle est proposée dans cet article.

Les associations de grands mammifères identifiées dans l'abri TR. 67 et les deux niveaux de la Grande Dune indiquent assez nettement un contexte écologique assez comparable, en l'occurrence un paysage en mosaïque de prairie-parc boisé, sous un climat tempéré. En effet, Aurochs et Cerf sont des marqueurs de ce type de milieu et de climat ; l'association avec un Cheval aux protocônes peu développés, caractère généralement considéré comme étant plutôt l'indice d'une forme caballine de contexte tempéré, va donc tout à fait dans le même sens. Pour TR. 67, la présence complémentaire du Mégalocéros n'est pas incongrue, cet animal étant couramment rencontré dans ce type de contexte écologique. Cette interprétation est renforcée par ailleurs par la présence dans TR. 67 du Hérisson, forme tempérée typique.

Pour le niveau D6 de la Grande Dune, les trois espèces : Aurochs, Cerf et Cheval, sont seules présentes.

Pour le niveau E2, le Morse s'ajoute à ces taxons. La présence de cet animal, qui évoquerait plutôt une ambiance bien plus boréale de par la région où on trouve l'espèce actuellement, n'est en fait pas forcément un indicateur de climat froid. En effet, des Morses ont parfois été signalés dans des périodes récentes au large des côtes de la Hollande et en Mer du Nord
(Pedersen, 1974). Il a aussi été signalé jusque dans le golfe de Gascogne (Duguy, 1986). Le climat au cours des phases tempérées du stade 5 postérieures à l'Eemien ayant été plus frais que l'actuel, cet animal peut donc trouver sa place au sein de l'accumulation osseuse de ce niveau sans constituer pour autant une aberration écologique.

En conclusion, les trois ensembles mammaliens du Rozel témoigneraient donc d'une série d'occupations humaines durant une période tempérée du début glaciaire Weichsélien, dans un environnement de type prairie parc-boisé.

\subsection{3 - Aspects taphonomiques et palethnologiques}

L'intérêt au Rozel de la présence d'une faune de grands mammifères pléistocènes retrouvés associés avec une industrie paléolithique réside de manière générale pour le Grand Ouest de la France dans le très faible nombre de gisements ayant justement livré ce type de matériel archéologique. Les raisons en sont essentiellement d'ordre sédimentaire, la matrice encaissant les dépôts d'ossements a dans beaucoup de cas constitué un milieu défavorable, soit par sa texture (sédiments grossiers très perméables aux eaux de percolation), soit par sa chimie (sédiments trop acides entraînant la dissolution minérale). Des conditions de sédimentation particulière ont donc permis au Rozel, dans l'abri TR. 67 mais surtout dans les deux niveaux de la Grande Dune, la préservation des ossements fossiles. Comme nous l'avons déjà signalé, la conservation des os de TR. 67 est globalement assez mauvaise, les os étant très souvent altérés, voire dans un état rendant impossible toute identification anatomique et spécifique. A contrario, les vestiges fauniques de la Grande Dune présentent un aspect très peu modifié, ceci étant lié au mode d'enfouissement probablement très rapide (formation de la dune), dans un sédiment fin très calcaire ayant servi à protéger les os de toute attaque post-dépositionnelle.

L'examen des stigmates taphonomiques a été effectué sur tous les ossements du Rozel, ce qui nous a permis de mettre en exergue deux agents taphonomiques majeurs, le premier lié à l'origine de l'accumulation osseuse, le second à l'évolution de ce dépôt (fig. 17 ; tab. 15). Il apparaît ainsi que plusieurs dizaines d'ossements, outre ceux quasiment retrouvés aux limites de la conservation, présentent des marques d'altération très nettes, surtout en TR. 67 , avec au moins quatre pièces très émoussées. Cet émoussé est clairement postérieur au dépôt initial, comme le montre par exemple un fragment d'os long d'Aurochs présentant une fracturation hélicoïdale dont toutes les arêtes sont polies (fig. 18-a). Ces dégradations témoignent assurément d'une très forte perte de vestiges osseux, difficile par contre à estimer.

Le second agent taphonomique mis en évidence sur les ossements du Rozel est décelable par l'importance des pièces portant des indices de traitement par l'Homme, avec au moins trente trois os présentant des profils de fracturation hélicoïdale, caractéristiques 
234

\begin{tabular}{|l|l|}
\hline Le Rozel & NR \\
\hline os très altérés & 36, dont 4 émoussés \\
\hline desquamation & 1 \\
\hline fracturation hélicoïdale & 33 dont : $\begin{array}{r}\text { 1 os avec encoche et contrecoup } \\
1 \text { os avec encoche, enlèvement médullaire et stries } \\
1 \text { os avec enlèvement médullaire } \\
1 \text { os avec point d'impact }\end{array}$ \\
\hline éclats osseux & 2 \\
\hline os avec stries & 5 \\
\hline os brûlés & 1 \\
\hline
\end{tabular}

Tab. 15 : Le Rozel - Inventaire des stigmates taphonomiques observés sur les ossements de grands mammifères. Tab. 15: Le Rozel - Taphonomic marks identified on large mammal bones.
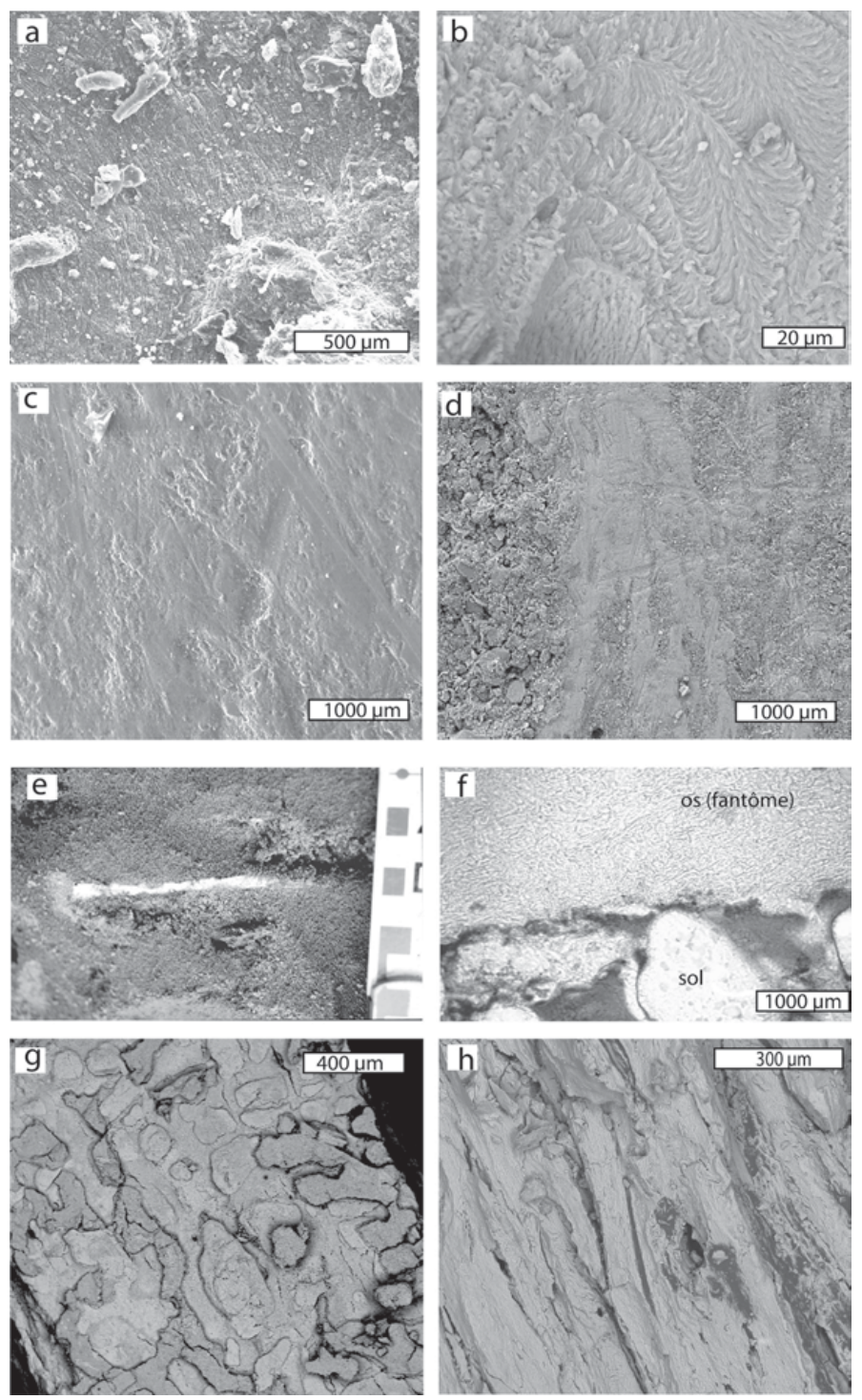

Fig. 17 : Le Rozel - a,b) os spongieux sec et cassant « non altéré » provenant probablement de la dune calcaire ; c,d) os polis provenant probablement de la dune calcaire : ce type de surface ne s'obtient pas par abrasion dunaire ou marine ; e) os altéré et pâteux dans le podzol D2c2 ; f-g) vues microscopiques de l'os altéré (f : pétrographie LN ; g-h : MEB) : notez l'aspect fibreux (collagène) du fantôme d'os et les gouttières de dissolution à la surface de l'os altéré (g)(photos B.Van Vliet-Lanoë).

Fig. $17:$ Le Rozel : a-b) spongy dry and friable bone, "unweathered" presumably from the calcareous dune; $c$-d) polished bone presumably from the calcareous dune: this kind of surface is not due to wind nor to marine abrasion; e) soft and weathered bone in the podsol D2c2; $f$ - $g$ ) microscopic views of the weathered bone $(f:$ petrography $L N ; g-h: S E M)$. Notice the fibrous aspect of the bone ghost (collagen) and solution scours at the bone surface ( $g$ )(pictures B.Van Vliet-Lanoë). 
d'une fracturation dynamique sur os frais, certaines de ces fracturations étant de plus associées avec d'autres stigmates d'origine anthropique (points d'impact, stries, ...). D'autres éléments portent uniquement des stries, deux fragments de diaphyses d'os longs sont de véritables éclats osseux résultant de la percussion des os longs pour en extraire la moelle, un seul os est brûlé. Aucune marque indiquant l'action d'un Carnivore en tant qu'agent accumulateur ou modificateur de l'état initial n'a été identifiée. Nous sommes donc en présence dans les trois niveaux considérés au Rozel d'une faune liée à une acquisition d'origine humaine, la question restant toutefois posée pour le Morse.

Les trois espèces dominantes semblent être concernées, mais c'est surtout l'Aurochs qui montre le plus nettement les indices d'activités de boucherie des Hommes, avec une certaine récurrence dans la fracturation des os longs (fig. 18-a, b et c), mais le Cerf est également concerné (fig. 18-d).
Les marques de découpe ont été repérées sur cinq os, elles indiquent des activités de boucherie de divers ordres. Un fragment de diaphyse de radius d'Aurochs (TR. 67) présente un profil de fracture dynamique. Il montre par ailleurs une zone d'encoche et un enlèvement médullaire témoignant du choc du percuteur ; des stries obliques sont présentes à la surface de cet os, témoignant de la récupération de la viande avant fracturation. Un talus d'Aurochs (Grande Dune, niveau D6) montre des stries au niveau de la zone d'insertion proximo-médiane, pouvant être attribuées à des marques de désarticulation. Une phalange intermédiaire d'Aurochs porte à son extrémité distale au niveau de l'insertion ligamentaire une double strie dont l'origine pourrait être la désarticulation, la récupération de tendon, voire le dépouillage (fig. 18-e). Un fragment de corps mandibulaire probablement de Cheval présente sur sa face linguale une très grande quantité de stries verticales pouvant traduire la récupération de la langue.

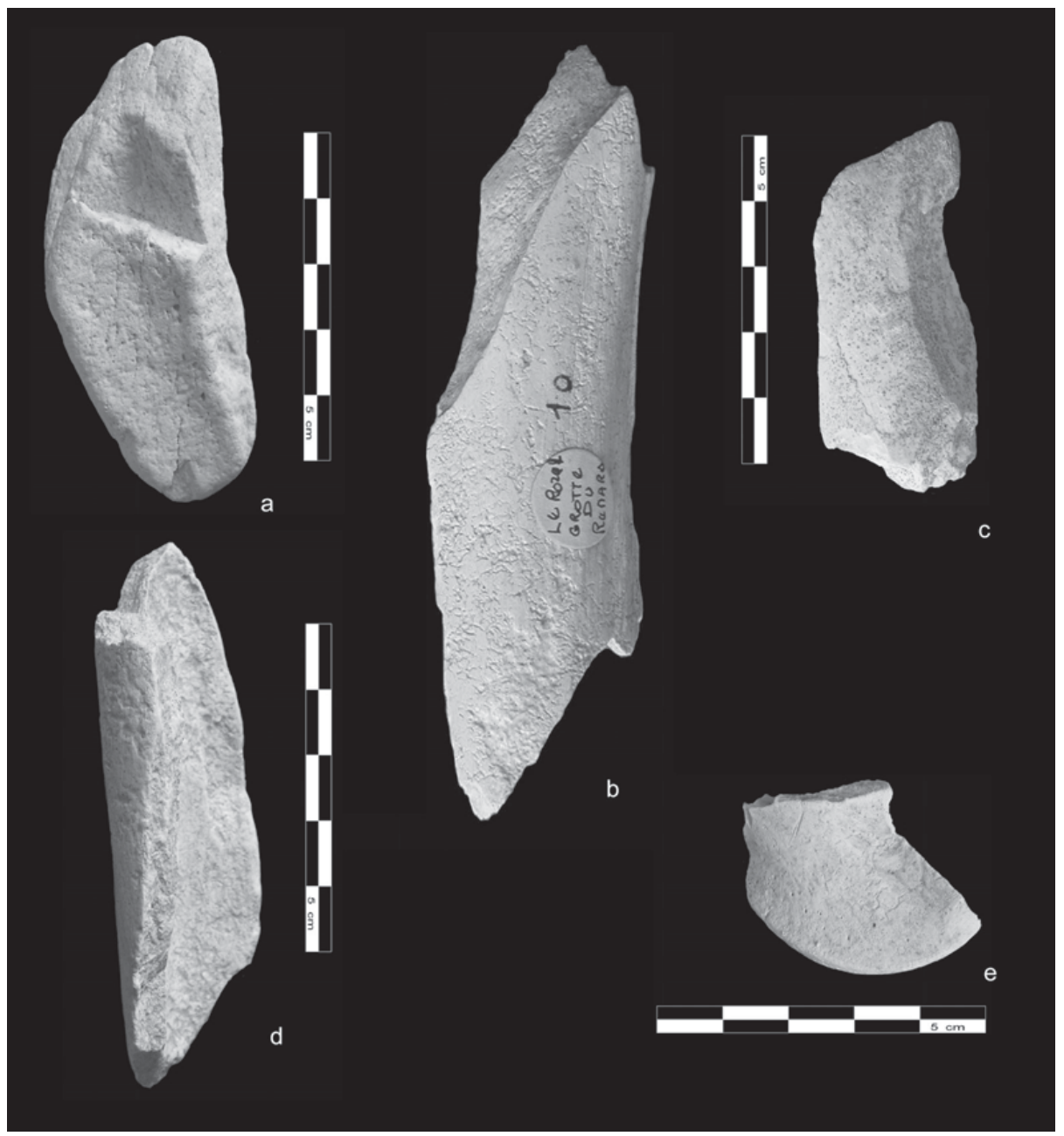

Fig. 18 : Le Rozel - Aurochs : (a) os long présentant une fracturation hélicoïdale et un émoussé très marqué (sans $\mathbf{n}^{\circ}$ ), vue médullaire, (b) radius présentant une fracturation hélicoïdale (TR 67, $n^{\circ} 10$ ), vue corticale, (c) éclat d'os long résultant d'une fracturation dynamique sur os frais (sans $\left.\mathrm{n}^{0}\right)$, vue corticale, (e) phalange intermédiaire présentant des stries de désarticulation (sans $\mathbf{n}^{\circ}$ ), vue latérale et Cerf : (d) os long présentant une fracturation hélicoïdale associée à des enlèvements médullaires (sans $\left.\mathbf{n}^{\circ}\right)$, vue médullaire.

Fig. 18: Le Rozel - Wild ox: (a) long bone with helicoidal fracture and blunted edges (without $n^{\circ}$ ), medullary view, (b) radius with helicoidal fracture (TR 67, $\left.n^{\circ} 10\right)$, cortical view, (c) bone flake resulting of dynamic impact on fresh bone (without $n^{\circ}$ ), cortical view, (e) second phalanx with cut marks (without $n^{\circ}$ ), lateral view and Red deer: (d) long bone with helicoidal fracture and medullary flakes (without $n^{\circ}$ ), medullary view. 
Un processus épineux de vertèbre thoracique porte une strie résultant du décharnement.

L'examen du profil de conservation des éléments anatomiques montre des disparités dans la représentation des parties squelettiques (tab. 16). Il apparaît ainsi une sous-représentation de la partie crânienne pour l'Aurochs, alors que l'on observe une répartition assez équitable du post-crânien. A l'inverse, le Cheval est surtout représenté par des éléments dentaires et peu de vestiges squelettiques. Le Cerf présente lui un profil intermédiaire entre les deux précédentes espèces. Il semble donc qu'il y ait eu soit une acquisition différentielle soit un traitement particulier adapté pour chaque animal. De par le faible nombre de pièces, il est difficile de postuler du mode d'acquisition de chaque individu identifié, mais les observations effectuées nous permettent d'envisager quelques pistes.

\begin{tabular}{|c|c|c|c|c|c|c|c|c|c|c|}
\hline \multirow{2}{*}{$\begin{array}{l}\text { Dénombrement par taxon } \\
\text { Éléments anatomiques }\end{array}$} & \multicolumn{2}{|c|}{ Aurochs } & \multicolumn{2}{|c|}{ Cheval } & \multicolumn{2}{|c|}{ Cerf } & \multicolumn{2}{|c|}{ Autres taxons } & \multicolumn{2}{|c|}{ Total } \\
\hline & NR & NMIc & NR & NMIc & NR & NMIc & NR & NMIc & NR & NMIc \\
\hline Bois de chute & & & & & 1 & 1 & & & 1 & 1 \\
\hline Bois & & & & & 6 & 1 & & & 6 & 1 \\
\hline & & & & & & & & & & \\
\hline Crâne & & & & & 2 & 1 & 3 & 1 & 5 & 2 \\
\hline Mandibules & 2 & 1 & 2 & 1 & 2 & 1 & 2 & 2 & 8 & 5 \\
\hline Déciduales & & & & & 1 & 1 & 1 & 1 & 2 & 2 \\
\hline \multicolumn{11}{|l|}{ Labiales } \\
\hline Jugales définitives supérieures & & & 5 & 1 & & & & & 5 & 1 \\
\hline Jugales définitives inférieures & 1 & 1 & 8 & 1 & & & & & 9 & 2 \\
\hline Dents indéterminés & 1 & 1 & & & 1 & 1 & & & 2 & 2 \\
\hline \multicolumn{11}{|l|}{ Hyoïdes } \\
\hline Sous-total crânien & 4 & 1 & 15 & 2 & 13 & 2 & 6 & 2 & 38 & 6 \\
\hline \multirow{2}{*}{\multicolumn{11}{|c|}{ Sternèbres }} \\
\hline & & & & & & & & & & \\
\hline Vertèbres & & & 5 & 1 & 7 & 2 & 5 & 1 & 17 & 3 \\
\hline \multicolumn{11}{|l|}{ Sacrum } \\
\hline Coxaux & 1 & 1 & & & & & 1 & 1 & 2 & 1 \\
\hline Côtes & 2 & 1 & & & 2 & 1 & 1 & 1 & 5 & 2 \\
\hline \multicolumn{11}{|l|}{ Cartilages costaux } \\
\hline & & & & & & & & & & \\
\hline Scapula & 2 & 1 & 1 & 1 & 1 & 1 & & & 4 & 3 \\
\hline Humérus & 7 & 3 & & & 1 & 1 & 2 & 1 & 10 & 4 \\
\hline Radius & 4 & 1 & 1 & 1 & & & 2 & 2 & 7 & 3 \\
\hline Ulna & & & & & 1 & 1 & & & 1 & 1 \\
\hline Radio-ulna & 1 & 1 & & & 1 & 1 & & & 2 & 2 \\
\hline Carpiens & 2 & 1 & & & & & & & 2 & 1 \\
\hline Métacarpiens & 1 & 1 & & & & & & & 1 & 1 \\
\hline & & & & & & & & & & \\
\hline Fémurs & 3 & 1 & & & & & 1 & 1 & 4 & 1 \\
\hline \multicolumn{11}{|l|}{ Patella } \\
\hline Tibia & 6 & 1 & & & 1 & 1 & & & 7 & 2 \\
\hline \multicolumn{11}{|l|}{ Fibula } \\
\hline \multicolumn{11}{|l|}{ Malléolaires } \\
\hline Tarsiens & 1 & 1 & & & & & & & 1 & 1 \\
\hline Métatarsiens & 3 & 2 & & & 3 & 1 & & & 6 & 3 \\
\hline Phalanges & 3 & 1 & & & 1 & 1 & & & 4 & 2 \\
\hline \multicolumn{11}{|l|}{ Sésamoïdes } \\
\hline Métapodiens & 1 & 1 & & & & & 1 & 1 & 2 & 1 \\
\hline Os longs & 15 & 1 & & & 6 & 1 & 10 & 2 & 31 & 2 \\
\hline Os & 3 & 1 & & & & & 212 & 2 & 215 & 2 \\
\hline Sous-total post-crânien & 55 & 3 & 7 & 1 & 24 & 2 & 235 & 1 & 321 & 7 \\
\hline Total & 59 & 3 & 22 & 2 & 37 & 3 & 241 & 3 & 359 & 11 \\
\hline
\end{tabular}

Tab. 16 : Le Rozel - Inventaire par élément anatomique et dénombrement des taxons de grands mammifères.

Tab. 16: Le Rozel - Counting and anatomical inventory of large mammals. 
Pour l'Aurochs, nous avons affaire à trois individus adultes, très probablement des mâles. Le déséquilibre observé pour la partie crânienne pourrait s'expliquer par le fait qu'il s'agit d'individus solitaires qui ont été abattus plus ou moins loin du site et dont seulement certaines parties squelettiques ont été ramenées, le crâne trop volumineux ayant été abandonné sur le lieu d'abattage. Pour le Cheval, le problème du crâne ne se pose pas et celui-ci a dû également être abattu aux alentours et ramené par quartier. Le Cerf semble lui par contre avoir été rapporté entier, surtout s'il s'agit de jeunes individus bien plus faciles à déplacer. Le traitement de boucherie semble avoir été assez systématique pour ces trois espèces, avec récupération du maximum de nourriture, la viande, la langue et la moelle osseuse.

La présence d'au moins un bois de chute en TR. 67 indique la collecte dans un but qui n'est pas directement alimentaire mais utilitaire, mais dont la fonction demeure inconnue (percuteur tendre, autre outil ?).

La saisonnalité enfin indique des occupations lors de périodes annuelles différentes, peut être la fin de l'hiver ou le début du printemps dans l'abri TR. 67, l'automne ou l'hiver pour les niveaux de la Grande Dune.

En conclusion, les éléments de la grande faune mammalienne laissent à penser que le site du Rozel a joué le rôle à plusieurs reprises de site de boucherie et/ou de halte de chasse passagère, avec une exploitation intensive des ressources alimentaires fournies par les animaux abattus aux environs du site.

\section{6 - LE MOBILIER LITHIQUE (D. C.)}

\section{1 - LES DIFFÉRENTS ENSEMBLES}

A la différence du matériel osseux, la quasi totalité du matériel lithique a été conservée, quelques objets faisant défaut, notamment le nucléus référencé D2-31 et le biface TR. 68 (fig. 19).

Plusieurs séries ont été signalées par F. Scuvée : l'ensemble issu de l'abri TR. 67, le plus fourni en nombre de pièces, l'assemblage provenant des couches E2-E3 et enfin des artefacts collectés en pied de falaise.

Parmi ceux-ci figurent quelques objets qui présentent des caractéristiques que nous ne retrouvons pas dans les deux assemblages collectés en place.

La pièce la plus intéressante, parce que rare en Cotentin, est un biface cordiforme figuré par F. Scuvée (1984), mais qui n'a pas été retrouvé.

Deux éclats Levallois affectés de retouches méritent notre attention. Ces enlèvements assez petits $(51 \mathrm{~mm}$ et $58 \mathrm{~mm}$ ), larges, et mince pour le premier, assez mince, pour le second semblent de « grande dimension » pour les séries collectées en place.

\section{L'assemblage lithique de la couche E2}

Le mobilier de la couche E2 (fig. 23-4 et 25-1 à 3 ; tab. 17) présente des états de surface variables. Si la plupart des artefacts présentent un «aspect frais », quelques pièces affichent un lustré caractéristique, soit lié à l'activité de l'eau, soit dû à l'éolisation.

La matière première mise en œuvre provient de cordons littoraux, comme l'attestent les pièces affectées de cortex qui présentent les coups d'ongles caractéristiques des galets d'origine marine.

La petite série ne comporte qu'un nucléus. Les enlèvements qui la constituent illustrent tous les stades de la chaîne opératoire (décorticage, mise en place des convexités, production d'enlèvements prédéterminés/prédéterminants et prédéterminés Levallois).

Le nucléus est géré selon la méthode Levallois (LR E2 ; L : 62, $1: 68$, e : 38, m : 140) (fig. 23-4). La lecture technologique des derniers enlèvements illustre une

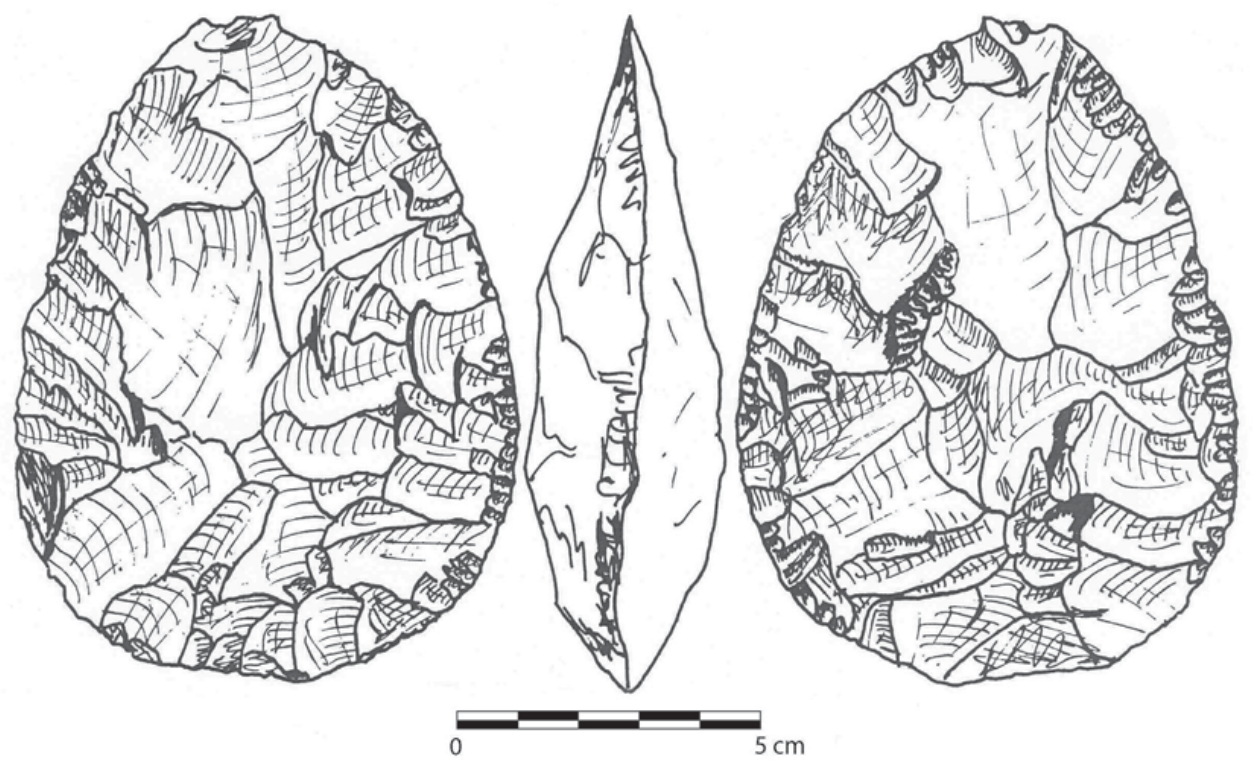

Fig. 19 : Biface cordiforme trouvé au pied de la dune (d'après F. Scuvée).

Fig. 19: Cordiform biface found at the base of the dune (after F. Scuvée). 


\begin{tabular}{|l|c|}
\hline Détermination & Silex \\
\hline Nucléus & 1 \\
\hline Eclat de décorticage à face entièrement corticale ou naturelle & 2 \\
\hline Eclat de décorticage à face 3/4 corticale ou naturelle & 1 \\
\hline Eclat de décorticage à face $1 / 2$ corticale ou naturelle & 4 \\
\hline Eclat débordant & 1 \\
\hline Eclat levallois & 7 \\
\hline Lame levallois & 2 \\
\hline Eclat de gestion de surface partiellement cortical & 1 \\
\hline Eclat indéterminé partiellement cortical & 2 \\
\hline Eclat laminaire partiellement cortical & 3 \\
\hline Esquille $<10 \mathrm{~mm}$ & 3 \\
\hline Esquille $<10 \mathrm{~mm}$ partiellement corticale & 3 \\
\hline Esquille $<10 \mathrm{~mm}$ corticale & 1 \\
\hline \multicolumn{2}{|l}{} \\
\hline Total & 31 \\
\hline
\end{tabular}

Tab. 17 : Décompte du mobilier lithique issu de la couche E2 de F. Scuvée.

Tab. 17: Details of stone artefacts from bed E2 of F. Scuvée

\begin{tabular}{|c|c|c|c|}
\hline Détermination & Silex & Quartz & Total \\
\hline Nucléus & 9 & 2 & 11 \\
\hline Eclat de décorticage à face entièrement corticale ou naturelle & 26 & & 26 \\
\hline Eclat de décorticage à $3 / 4$ de face corticale ou naturelle & 17 & & 17 \\
\hline Eclat de décorticage à $1 / 2$ face corticale ou naturelle & 27 & 1 & 28 \\
\hline Eclat d'aménagement & 20 & & 20 \\
\hline Eclat d'aménagement partiellement cortical & 11 & & 11 \\
\hline Eclat d'aménagement de convexité latérale & 2 & & 2 \\
\hline Eclat d'aménagement de convexité latérale partiellement cortical & 28 & & 28 \\
\hline Eclat débordant & 2 & & 2 \\
\hline Eclat ou lame Levallois & 47 & & 47 \\
\hline Pointe pseudo-levallois & 2 & & 2 \\
\hline Eclat de gestion de surface & 31 & 1 & 32 \\
\hline Eclat de gestion de surface partiellement cortical & 23 & 2 & 25 \\
\hline Eclat kombéwa & 3 & & 3 \\
\hline Eclat indéterminé & 30 & 4 & 34 \\
\hline Eclat indéterminé partiellement cortical & 13 & 7 & 20 \\
\hline Eclat laminaire & 4 & & 4 \\
\hline Eclat laminaire partiellement cortical & 11 & & 11 \\
\hline Lame ou lamelle de gestion de surface & 20 & & 20 \\
\hline Lame ou lamelle de gestion de surface partiellement corticale & 5 & & 5 \\
\hline Lame d'initialisation à dièdre naturel & 1 & & 1 \\
\hline Lame à crête frontale & 4 & & 4 \\
\hline Lame à crête latérale & 1 & & 1 \\
\hline Lame ou lamelle à arêtes parallèles & 11 & & 11 \\
\hline Eclat de façonnage? & 1 & & 1 \\
\hline Esquille $<10 \mathrm{~mm}$ & 114 & 7 & 121 \\
\hline Esquille $<10 \mathrm{~mm}$ partiellement corticale & 12 & & 12 \\
\hline Esquille $<10 \mathrm{~mm}$ corticale & 14 & & 14 \\
\hline Petit élément $<15 \mathrm{~mm}$ & 135 & & 135 \\
\hline Petit élément $<15 \mathrm{~mm}$ partiellement cortical & 59 & & 59 \\
\hline Petit élément $<15 \mathrm{~mm}$ cortical & 14 & & 14 \\
\hline Débris & 5 & 1 & 6 \\
\hline Total & 702 & 25 & 727 \\
\hline
\end{tabular}

Tab. 18 : Composition de l'assemblage lithique de l'abri TR.67.

Tab. 18: Composition of the lithic assemblage from shelter TR 67. 
exploitation bipolaire de la surface Levallois conduite depuis deux plans de frappe opposés. Les convexités latérales sont entretenues par une série de petits éclats.

Le dernier éclat d'aménagement affecte la surface de débitage en raison d'une inclusion qui génère un enlèvement épais qui rebrousse. Malgré un potentiel en matière d'œuvre encore important (140 g), le nucléus est abandonné

Les convexités distales sont entretenues par la gestion bipolaire opposée du débitage.

Les derniers enlèvements débités sont de petites dimensions et très larges.

Une seule pièce comporte une retouche régulière, un racloir double convergent ou pointe moustérienne aménagé sur un éclat Levallois triangulaire (fig. 24-2). Deux éclats laminaires sont affectés d'une encoche.

Les modules des enlèvements entiers n'excèdent pas $60 \mathrm{~mm}$. Les artefacts sont majoritairement larges et assez longs, et assez épais, assez minces et minces.

Bien que la série ne soit pas statistiquement représentative, le débitage apparaît orienté vers la production d'éclats prédéterminés, notamment Levallois et d'enlèvements allongés (lame Levallois et éclats laminaires).

Le débitage s'effectue au percuteur dur. L'analyse atteste d'une préparation des plans de frappe soignée tout particulièrement pour les enlèvements Levallois et laminaires (facettage, abrasion).

Un éclat Levallois récurrent centripète présente de grandes similitudes physiques et techniques avec l'assemblage collecté dans l'abri TR. 67. Aucun raccord n'a été possible entre les deux ensembles.

\section{L'assemblage lithique issu de l'abri TR. 67}

La série lithique (fig. 22, 23-1 à 3, 24, 25-3 à 8 et 26 ; tab. 18) est constituée de 727 pièces dont l'essentiel regroupe des éclats corticaux (n: 172, soit 9,8\%) et des esquilles et petits éléments inférieurs à $15 \mathrm{~mm}$ (n : 355 , soit $48,8 \%$ ) (tab. 18).

Cette proportion (58,5\% des artefacts) atteste d'une production dans l'abri et/ou à proximité immédiate.
La production se caractérise par la coexistence de deux chaînes opératoires structurées en fonction de schémas de modalités différentes.

\section{2 - LES STRATÉGIES D'EXPLOITATION DES MATIÈRES PREMIÈRES}

La série issue de l'abri du Rozel est une industrie à composante lithologique mixte, fondée sur la mise en œuvre du silex issu des cordons littoraux et du quartz de filon qui rythme la falaise de schiste (fig. 20).

La représentativité du silex $(96,6 \%)$ au sein de l'assemblage est aléatoire, du fait que seuls quelques artefacts en quartz ont été prélevés, comme l'indique le rapport de fouille de 1969: «il nous a semblé utile de noter et conserver quelques exemplaires de quartz ayant vraisemblablement servi, leur nombre ayant été infiniment plus grand dans les couches explorées (p. 8 et 9)».

Il eut été intéressant de tenter une analyse technologique comparée des deux ensembles lithologiques.

Les rognons de silex exploités par les préhistoriques présentent les stigmates caractéristiques des galets marins, nettoyés de leur gangue, affectés de coups d'ongles... Leur dimension s'avère modeste, vraisemblablement en raison d'une accessibilité limitée aux cordons littoraux recouverts par les massifs dunaires. Ces nodules devaient donc être présents dans un environnement proche, au maximum quelques kilomètres.

La taille de ces rognons à leur état ultime d'exhaustion (entre $68 \mathrm{~mm}$ et $28 \mathrm{~mm}$ ) et la longueur maximale des enlèvements $(78 \mathrm{~mm}$ ) laissent supposer l'exploitation de galets de petites dimensions (tab. 19).

Le quartz de filon était présent dans l'abri même et vraisemblablement présent à même le sol en raison de l'altération du schiste notamment à l'interface entre les deux roches. Cette matière première se présente sous forme de «parallélépipèdes » relativement réguliers dont les angles approchent les $90^{\circ}$.

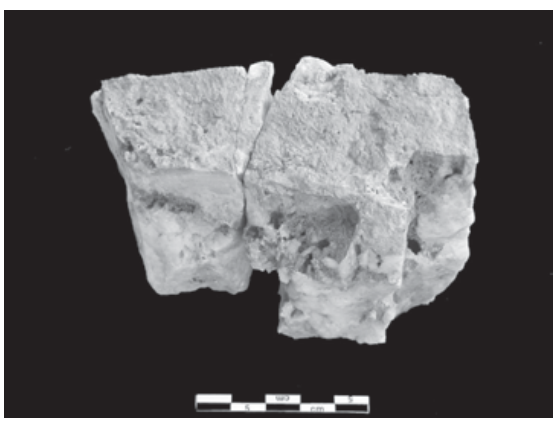

Fig. 20 : Veine de quartz filonien à l'intérieur de l'abri et remontage de deux blocs de quartz et d'un éclat (clichés D. Cliquet). Fig. 20: Quartz vein in the shelter and two blocks of broken quartz (photo D. Cliquet). 


\begin{tabular}{|l|l|l|l|c|}
\hline & L & l & e & masse (g) \\
\hline Quartz G1 134 & 79 & 68 & 61 & 440 \\
\hline Quartz G1. 127 & 66 & 64,5 & 54 & 295 \\
\hline Silex TR. 77 & 57 & 54 & 27,5 & 80 \\
\hline Silex TR.89 & 51,5 & 41 & 25,5 & 60 \\
\hline Silex H1. 40 & 46 & 48 & 19 & 35 \\
\hline Silex TR. 76 & 52 & 47,5 & 16 & 30 \\
\hline Silex D2. 32 & 43,5 & 30,5 & 20 & 15 \\
\hline Silex B2. 6 & 39,5 & 39 & 13,5 & 15 \\
\hline Silex TR. 20 & 26 & 37 & 15 & 15 \\
\hline Silex G1. 156 & 29 & 28 & 8,5 & 10 \\
\hline
\end{tabular}

Tab. 19 : Dimensions et masses des nucléus à leur état ultime d'exhaustion. Tab. 19: Size and weight of cores.

\section{3 - LES CHAÎNES OPÉRATOIRES}

\subsection{1 - Chaînes opératoires à éclats}

La production d'éclats apparaît dominante au sein de la série $(66,5 \%$ des produits de plein débitage).

\section{L'utilisation du quartz : un débitage direct, exploi-} tant les convexités naturelles

Les deux blocs de quartz exploités, de forme grossièrement parallélépipédique, proviennent d'une veine et se raccordent entre eux (fig. 20 et 21).

Les angles définis par les intersections des faces légèrement inférieurs à $90^{\circ}$ ont permis l'exploitation indépendante de trois faces du premier bloc et de deux des faces du second.

La production peu poussée s'effectue sans aménagement des surfaces de débitage, ni des surfaces de plan de frappe. La lecture des négatifs témoigne de l'obtention d'enlèvements majoritairement très larges ou larges, en raison du caractère peu marqué des convexités naturelles des blocs.

Le potentiel en matière première reste important au moment de l'abandon du bloc, comme en témoignent : le faible nombre d'enlèvements pratiqués au percuteur dur sur chacun des supports de débitage (respectivement 7 et 4 éclats), et les dimensions et la masse des nucléus à leur état ultime d'exploitation (tab. 19).

\section{La gestion de surface des galets de silex}

- Nucléus TR. 77 (fig. 22-3)

Les vestiges d'enlèvements observés sur le nucléus TR. 77 illustrent une tentative d'exploitation d'un petit galet littoral préalablement affecté par le gel.

Après la production d'au moins deux éclats effectués depuis une surface de plan de frappe préparée, le tailleur réaménage, par le débitage de deux enlèvements, la surface de plan de frappe.

C'est vraisemblablement à cette occasion qu'un éclat de gel se détache du galet. L'onde de choc suit une fissure faite par le gel.
Le tailleur retourne le bloc et utilise le négatif de cet éclat comme plan de frappe. Les vestiges d'un petit éclat sont affectés par le détachement accidentel d'un gros éclat dû au gel (ombilic bien marqué).

Ce nouvel accident mène à l'abandon du galet malgré une relative quantité de matière d'œuvre disponible $(80 \mathrm{~g})$.

\section{- Nucléus TR. 89 (fig. 22-1)}

La lecture de cette pièce fragmentée apparaît peu aisée. L'exploitation s'effectue selon une gestion centripète (?) plutôt sécante qui n' autorise pas la poursuite du débitage, en raison d'un manque de matière, à la différence des autres nucléus qui sont gérés selon un schéma parallèle.

La pièce est abandonnée après une tentative de réaménagement de la surface de débitage effectuée depuis une surface de plan de frappe. Un réaménagement du nucléus ne serait pas rentable, bien que le potentiel en matière première reste "relativement important" $(60 \mathrm{~g})$.

\section{* Gestion de surface Levallois bipolaire}

\section{- Nucléus H1.40 (fig. 23-1)}

L'analyse des négatifs des derniers enlèvements atteste d'une exploitation récurrente unipolaire conduite depuis une surface de plan de frappe, succédant au débitage d'un grand éclat envahissant produit depuis une surface de plan de frappe opposée.

Le raccord de deux éclats d'aménagement de convexités latérales montre un entretien de ces convexités par le débitage de petits éclats centripètes à talons corticaux.

La convexité distale est aménagée par un grand enlèvement outrepassé.

La lecture technologique de ce support de débitage témoigne d'un débitage envahissant (deux grands éclats bipolaires) poursuivi par une exploitation récurrente unipolaire.

Les derniers enlèvements unipolaires rebroussent malgré l'existence de convexités. Une tentative 

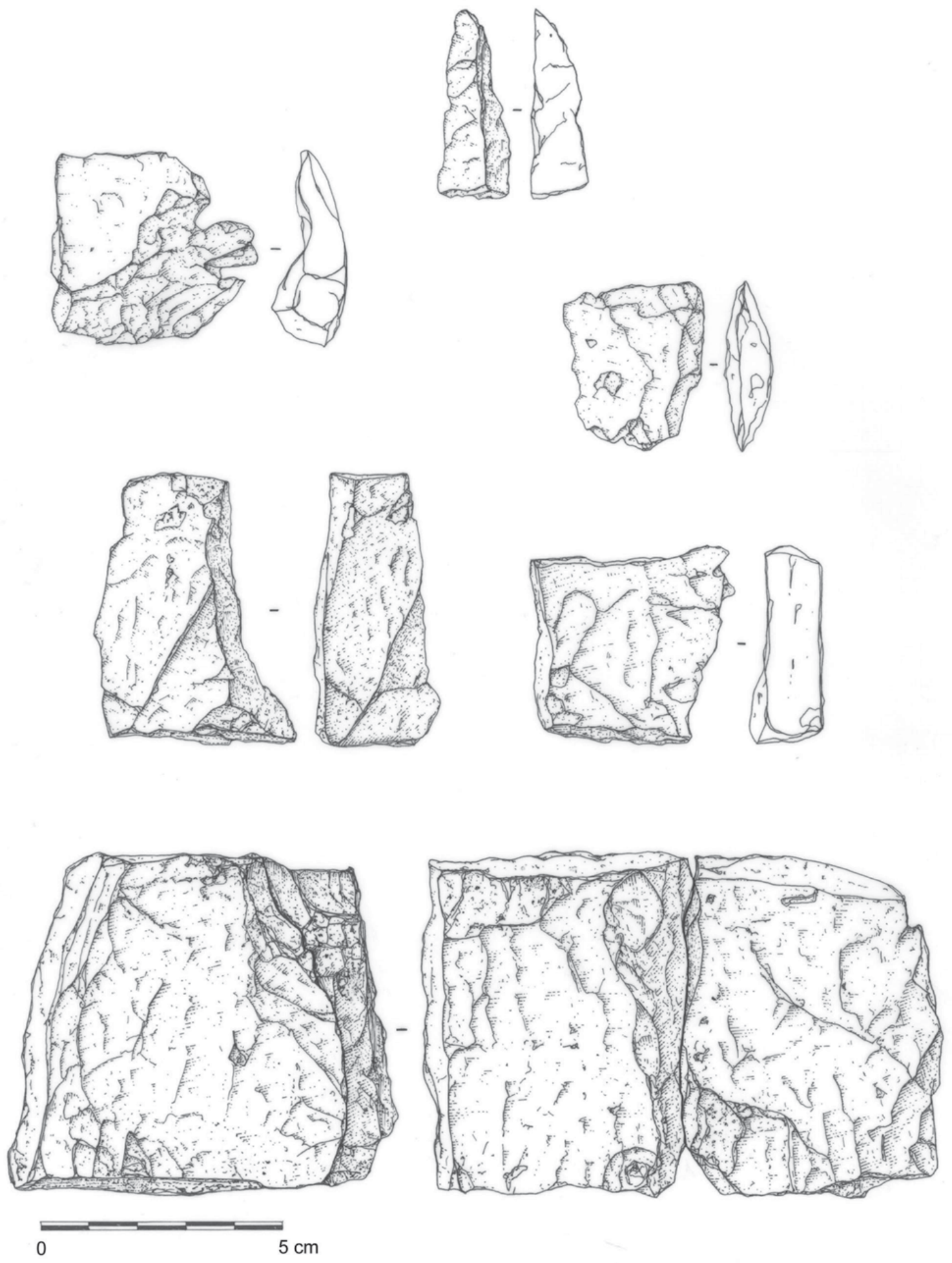

Fig. 21 : Production d'éclats en quartz de filon (Dessin P. Alix). Fig. 21: Product of broken vein quartz (Drawing: P. Alix). 

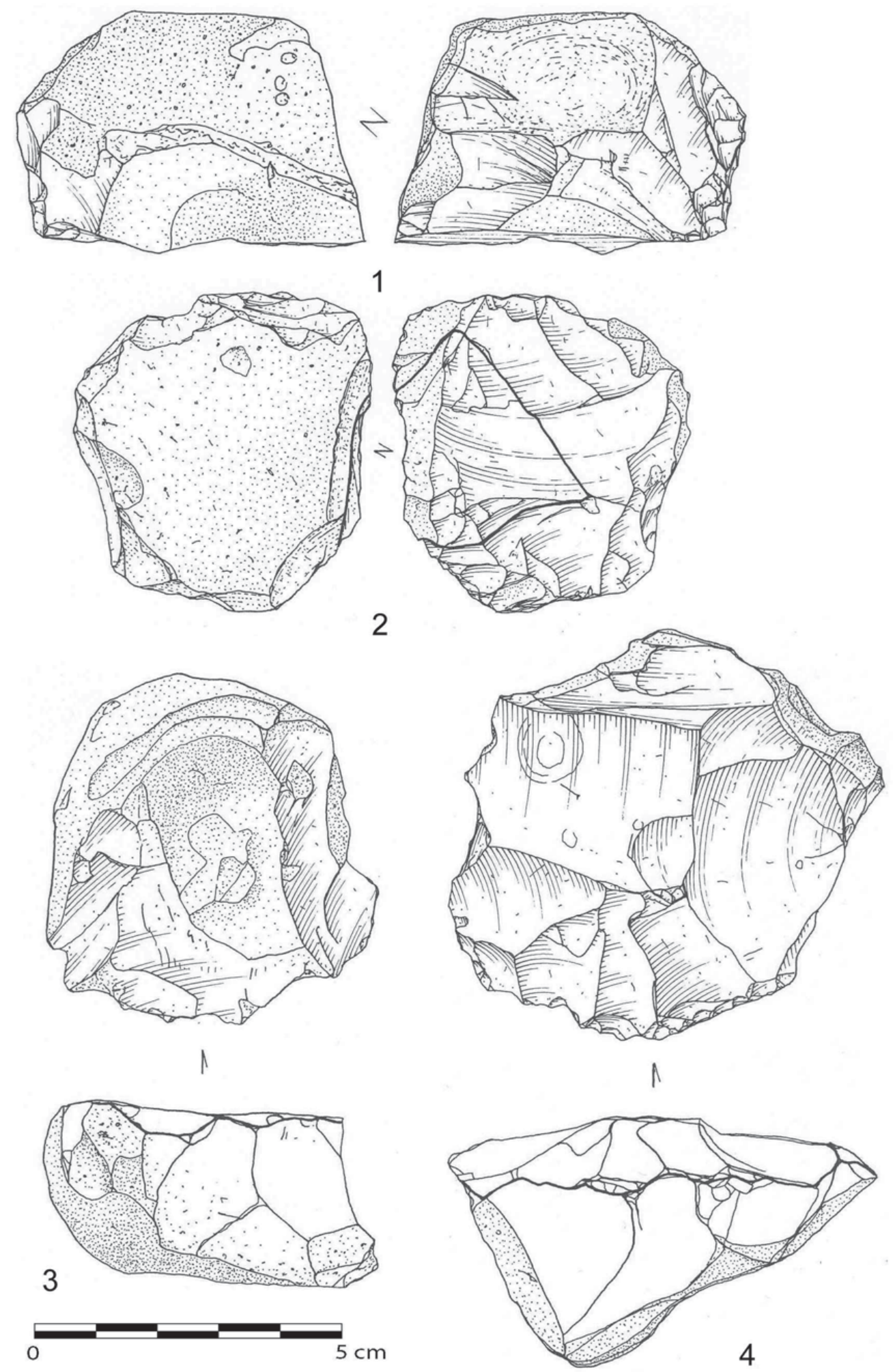

Fig. 22 : Abri TR 67 : Nucléus de gestion de surface : 1, 2 et 3 ; Grande Dune : Nucléus de gestion de surface : 4 (Dessins P. Alix). Fig. 22: Shelter TR67 working of the core surface (1,2 and 3); Grande Dune (4) (Drawing: P. Alix). 
d'aménagement de convexité latérale rebrousse; le nucléus est alors abandonné, faute de matière d'œuvre (35 g). Les derniers produits obtenus sont de petites dimensions, larges à laminaires.

Les deux éclats préférentiels étaient vraisemblablement assez petits et larges (?).

* Gestion de surface Levallois bipolaire croisée alternative (nucléus TR. 76) (fig. 22-2)

Le nucléus TR. 76 témoigne d'une gestion récurrente de la surface de débitage exploitée depuis des plans de frappe qui affectent toute la périphérie de la pièce.

L'exploitation est conduite depuis deux plans de frappe opposés qui aménagent les convexités latérales de la séquence suivante menée perpendiculairement à la première.

Cependant, les convexités peu marquées génèrent des petits éclats plats, très larges, qui rebroussent, comme l'atteste le dernier enlèvement débité avant abandon du nucléus.

La production n'est pas poursuivie en raison de la réduction du support de débitage ( $30 \mathrm{~g}$ ), notamment de son épaisseur.

* Gestion de surface Levallois bipolaire orthogonale alternative (nucléus TR. 20) (fig. 23-3)

La lecture technologique des négatifs d'enlèvements portés par la surface de débitage, ainsi qu'un raccord d'éclat attestent d'une exploitation de la surface Levallois par une gestion bipolaire orthogonale alternative.

Le tailleur conduit le débitage depuis deux surfaces de plan de frappe orthogonales sur un nucléus large et court, à son état ultime d'exhaustion.

L'exploitation s'effectue depuis une surface de plan de frappe, puis depuis la surface de plan de frappe perpendiculaire. Ce processus permet l'entretien des convexités latérales par séquences de production d'éclats alternatives.

L'étroitesse de la surface Levallois évite l'entretien de la convexité distale. Le débitage est poursuivi jusqu'à quasi épuisement de la matière d'œuvre. A son ultime état d'exhaustion, le nucléus mesure $26 \mathrm{~mm}$ par $37 \mathrm{~mm}$, pour une masse de $15 \mathrm{~g}$.

Les éclats prédéterminés obtenus sont de petites dimensions, très larges à assez longs. L'analyse de ce nucléus traduit la volonté du tailleur de produire des micro-éclats Levallois.

* Gestion de surface Levallois bipolaire orthogonale (nucléus G1. 156) (fig. 23-4)

Ce nucléus illustre une gestion bipolaire orthogonale conduite depuis deux surfaces de plan de frappe, jusqu'à exhaustion complète de la matière d'œuvre (10 g).

Les convexités sont entretenues par le débitage orthogonal et par de petits enlèvements centripètes.

Les derniers enlèvements produits sont de très petites dimensions, très larges et larges et traduisent de nouveau la recherche de petits éclats prédéterminés.

\section{* Gestion de surface Levallois centripète}

\section{- Nucléus B2.6 (fig. 23-2)}

La lecture des derniers enlèvements effectués atteste d'une gestion centripète conduite depuis des surfaces de plan de frappe quasi périphériques, succédant à une séquence de débitage unipolaire récurrente.

Les vestiges d'éclats correspondant à cette séquence de débitage montrent une gestion des convexités latérales par récurrence et l'entretien de la convexité distale par de courts éclats bipolaires débités depuis une surface de plan de frappe opposée.

A son état ultime d'exhaustion, les derniers négatifs d'éclats illustrent un débitage centripète mené depuis des surfaces de plan de frappe préparées. Ces éclats de petites dimensions, très larges et larges rebroussent et achèvent l'exploitation du nucléus ( $15 \mathrm{~g}$ ).

Une nouvelle fois, le tailleur semble avoir voulu optimiser la production de son nucléus par le débitage de micro-éclats prédéterminés.

\subsection{2 - Chaîne opératoire à lames}

Deux nucléus témoignent de la recherche de produits longs. Cependant un seul nucléus nous est parvenu.

\section{- Nucléus D2. 31 (non retrouvé) (fig. 23-6)}

Le dessin et le descriptif fournis dans la publication (Scuvée \& Vérague, 1984) indiquent une production mixte :

- laminaire semi tournante conduite depuis un seul plan de frappe préparé. Le débitage récurrent semble entretenir des convexités latérales. La convexité distale ne nécessite pas d'aménagement particulier, en raison de la morphologie du nucléus de forme « pyramidale ». Les produits laminaires obtenus sont de gestion unipolaire, laminaire, à arêtes parallèles.

- unipolaire destinée à l'obtention d'éclats laminaires. Le tailleur exploite une surface depuis la table laminaire précédemment exploitée. Les enlèvements unipolaires sont assez longs, cependant le concept semble laminaire (?).

\section{- Nucléus D2. 32 (fig. 23-5)}

La lecture technologique de ce nucléus témoigne d'une volonté de produire des lames depuis une surface de plan de frappe préparée.

Les convexités latérales sont aménagées par le principe de récurrence conduit selon un débitage unipolaire semi tournant. Les vestiges d'éclats latéraux indiquent une préparation initiale de la surface de débitage par l'élaboration d'une crête à deux versants.

L'exploitation de ce nucléus apparaît donc conduite selon les principes qui régissent la production laminaire de "type paléolithique supérieur », définie par une « initialisation » par crête frontale à double versant, extension de la table laminaire par un débitage récurrent unipolaire conduit sur un nucléus court, ce qui évite l'entretien de la convexité distale. 

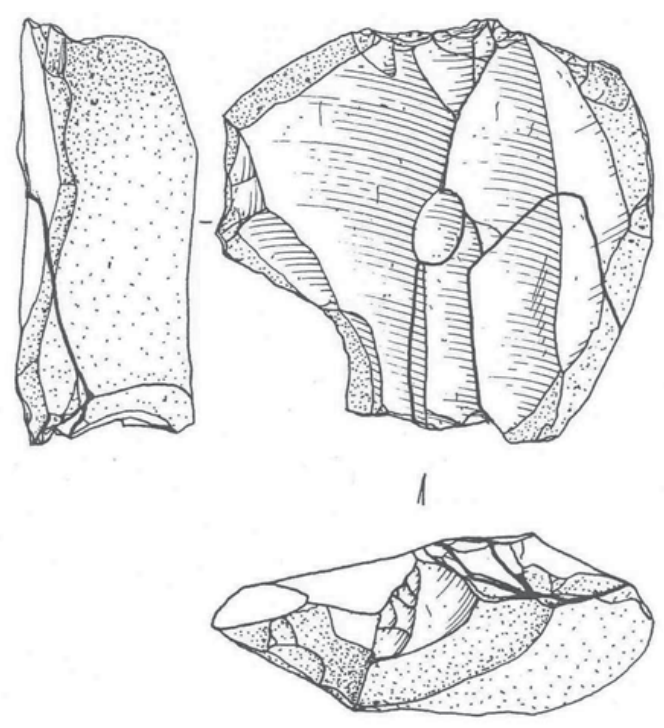

1
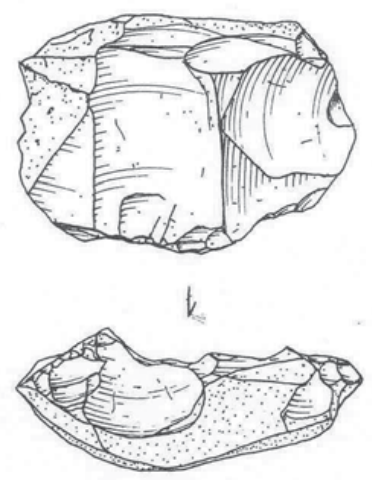

3

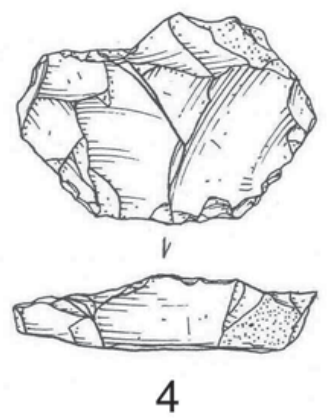

$5 \mathrm{~cm}$

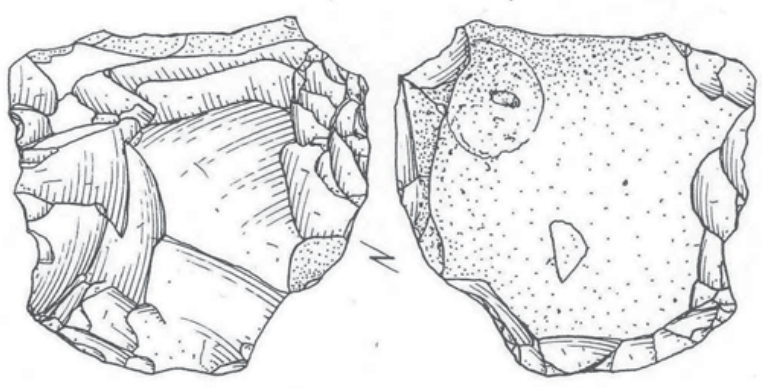

2
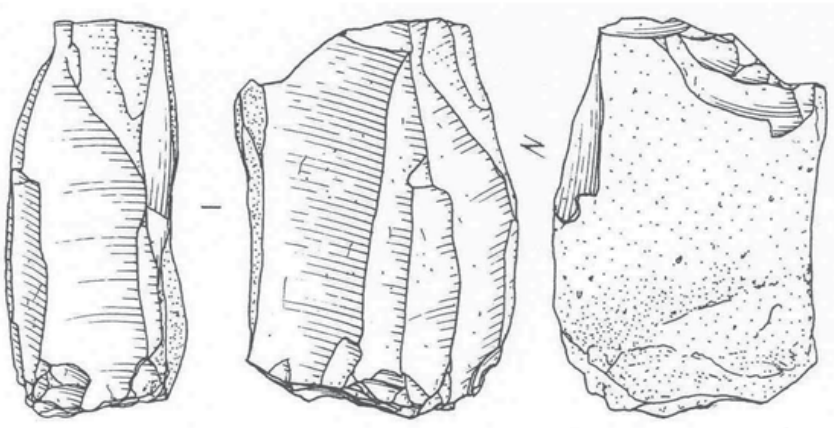

1

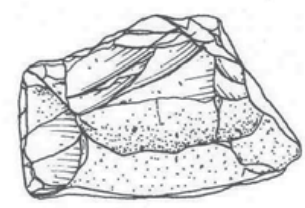

5

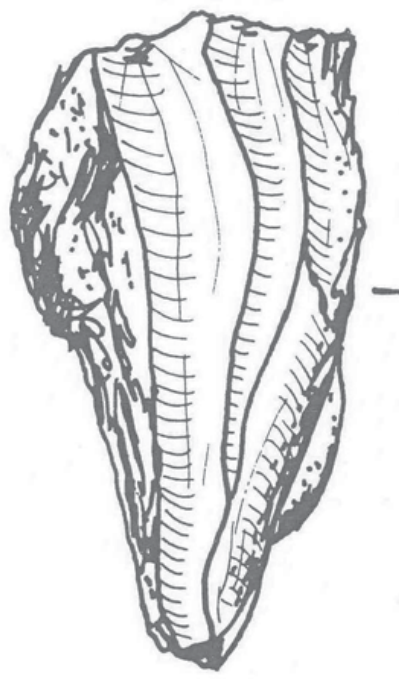

6

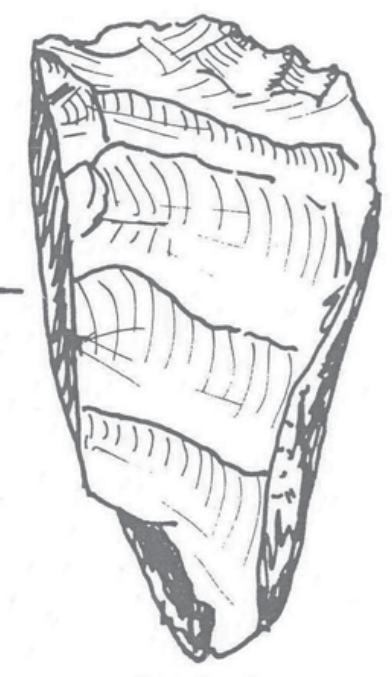

Fig. 23 : Abri TR 67 : Nucléus Levallois : 1, 2, 3 et 4 ; nucléus laminaires : 5 et 6 (Dessins P. Alix : 1 à 5 ; 6 d'après Scuvée, 1984). Fig. 23: Shelter TR57 Levallois core (1,2,3,4); laminar core (5 and 6) (Drawings P.Alix 1-5; 6 after Scuvée, 1984). 
Les derniers produits obtenus sont des éclats longs et assez longs de petites dimensions, cependant le concept est laminaire.

\section{4 - UNE PRODUCTION D'ÉCLATS ET DE LAMES}

La confrontation des données fournies par l'analyse des données morphométriques des négatifs d'enlèvements et des éléments débités permet de mettre en évidence une corrélation étroite entre la représentation des négatifs d'enlèvements mesurés sur les nucléus et l'ensemble des enlèvements collectés (tab. 20).

Les très petites et petites pièces $(<40 \mathrm{~mm})$ représentent $80 \%$ des négatifs observés sur les nucléus à leur état ultime d'exhaustion et presque $77 \%$ du mobilier collecté.

Cet « équilibre»se retrouve pour la catégorie des pièces de dimension assez petite avec environ $20 \%$ des négatifs et des enlèvements.

Seuls les artefacts de moyenne dimension $(3,6 \%)$ n'ont pas leur pendant sur les nucléus, en raison de l'intensité d'exploitation des nodules.

Les très petites pièces $(<20 \mathrm{~mm})$ et petites (entre 20 $\mathrm{mm}$ et $40 \mathrm{~mm}$ ) comportent essentiellement des éclats de décorticage, d'aménagement ou indéterminés. De nombreux enlèvements de petite dimension illustrent le plein débitage, notamment avec la production d'éclats Levallois, Kombéwa, de lames obtenues par gestion de surface et de lames à arêtes parallèles dues à un débitage semi-tournant $(46 \%$ des pièces de petite dimension).

Cette confrontation s'avère moins démonstrative pour les modules établis à partir du rapport longueur / largeur.

Les négatifs d'enlèvements mesurés sur les nucléus attestent d'une bonne représentation des éclats très larges $(45 \%)$ et assez longs ( $25 \%)$, aux dépens des enlèvements longs (10\%), larges (10\%) et laminaires.

Les artefacts mesurables sont dominés par les éclats larges $(40 \%)$ et très larges $(35 \%)$; viennent ensuite les enlèvements assez longs (16\%), longs (7,4 \%), laminaires $(2 \%)$ et les lames (moins de $1 \%$ ).

Cette dichotomie s'explique par la variété des concepts de mise en œuvre des matières premières : débitage direct, production d'enlèvements prédéterminés Levallois et laminaires exploitant une surface et de lames à arêtes parallèles gérant un volume.

Les éclats de décorticage s'avèrent principalement larges et très larges ( $85 \%)$, comme les éclats d'aménagement $(100 \%)$ à la différence de ce qu'on observe pour les enlèvements d'entretien (gestion des surfaces de débitage et des «tables» laminaires). Parmi ces

\begin{tabular}{|l|c|c|c|}
\hline quartz G1. 127 & L & Module & Rapport L/l \\
\hline 38 & Petit & $\mathrm{AL}$ \\
\cline { 2 - 4 } & 33 & Petit & large \\
\cline { 2 - 4 } & 23 & Petit & Très large \\
\cline { 2 - 4 } & 13 & Très Petit & Très large \\
\hline
\end{tabular}

\begin{tabular}{|l|c|c|c|}
\hline quartz G1.134 & L & Module & Rapport L// \\
\cline { 2 - 4 } & 51 & Assez Petit & large \\
\cline { 2 - 4 } & 38 & Petit & Très large \\
\cline { 2 - 4 } & 18 & Très Petit & Très large \\
\cline { 2 - 4 } & 15 & Très Petit & large \\
\cline { 2 - 4 } & 11 & Très Petit & Très large \\
\cline { 2 - 4 } & 9,5 & Très Petit & Très large \\
\hline
\end{tabular}

\begin{tabular}{|l|c|c|c|}
\hline Silex TR. 77 & L & Module & Rapport L// \\
\hline \multirow{4}{*}{} & 42 & Assez Petit & large \\
\cline { 2 - 4 } & 19 & Très Petit & Très large \\
\cline { 2 - 4 } & 12,5 & Très Petit & Très large \\
\cline { 2 - 4 } & 10 & Très Petit & Très large \\
\hline
\end{tabular}

\begin{tabular}{|l|c|c|c|}
\hline Silex TR. 76 & L & Module & Rapport L/l \\
\hline \multirow{4}{*}{} & 30 & Petit & Très large \\
\cline { 2 - 4 } & 20 & Petit & Très large \\
\cline { 2 - 4 } & 17 & Très Petit & Très large \\
\hline
\end{tabular}

\begin{tabular}{|l|c|c|c|}
\hline Silex H1.40 & L & Module & Rapport L// \\
\hline \multirow{4}{*}{} & 46 & Assez Petit & Laminaire \\
\cline { 2 - 4 } & 28 & Petit & large \\
\cline { 2 - 4 } & 26 & Petit & Long \\
\hline 24 & Petit & Long \\
\cline { 2 - 4 } & 24 & Petit & Assez Long \\
\cline { 2 - 4 } & 19 & Très Petit & Assez Long \\
\hline
\end{tabular}

\begin{tabular}{|l|r|c|c|}
\hline Silex G1. 156 & L & Module & Rapport L/l \\
\hline \multirow{4}{*}{} & 12 & Très Petit & Très large \\
\cline { 2 - 4 } & 8 & Très Petit & large \\
\cline { 2 - 4 } & 17 & Très Petit & large \\
\hline
\end{tabular}

\begin{tabular}{|l|c|c|c|}
\hline Silex D2.32 & L & Module & Rapport L/I \\
\hline \multirow{4}{*}{} & 43,5 & Assez Petit & Long \\
\cline { 2 - 4 } & 41,5 & Assez Petit & Assez Long \\
\cline { 2 - 4 } & 25 & Petit & Assez Long \\
\hline
\end{tabular}

\begin{tabular}{|l|c|c|c|}
\hline Silex TR. 20 & L & Module & Rapport L// \\
\cline { 2 - 4 } & 22,5 & Petit & Assez Long \\
\cline { 2 - 4 } & 14 & Très Petit & Très large \\
\hline \multirow{4}{|c|}{ Silex B2. 6 } & L & Module & Rapport L// \\
\hline \multirow{5}{*}{} & 10,5 & Très Petit & Très large \\
\cline { 2 - 4 } & 13 & Très Petit & large \\
\cline { 2 - 4 } & 17 & Très Petit & Très large \\
\cline { 2 - 4 } & 15 & Très Petit & Très large \\
\cline { 2 - 4 } & 25 & Petit & large \\
\cline { 2 - 4 }
\end{tabular}

Tab. 20 : Dimensions et modules des derniers enlèvements débités sur les nucléus (d'après les négatifs mesurables).

Tab. 20: Sizes and volumes of the last flakes knapped on cores. 
pièces, les éclats larges (54\%) et assez longs (23\%) dominent les enlèvements très larges $(8,6 \%)$, laminaires $(8,6 \%)$ et longs $(5,7 \%)$.

La plupart des éclats indéterminés sont très larges $(59 \%)$ et larges $(32 \%)$.

Enfin, parmi les pièces de plein débitage, les formes très larges $(33 \%)$, larges $(25 \%)$ et assez longues $(25 \%)$ dominent les enlèvements longs (13\%), laminaires $(2 \%)$ et les lames morphométriques $(1 \%)$.

Les artefacts sont principalement minces (40\%), assez minces (28\%) et assez épais (20\%). Les pièces très minces $(8 \%)$, épaisses $(4 \%)$ et très épaisses (moins de $1 \%$ ) sont plus rares.

Cette proportion se retrouve globalement au sein des produits de plein débitage : minces (50\%), assez minces $(22 \%)$, assez épais $(15 \%)$; les enlèvements très minces $(10 \%)$ et épais $(4 \%)$ étant moins bien représentés.

Il aurait été intéressant d'effectuer une comparaison avec les productions en quartz, mais les quelques pièces collectées ne sont statistiquement pas représentatives ; seul un échantillonnage ayant été collecté lors de la fouille. De ce fait, les quartz ne représentent plus que 3,4\% du mobilier collecté.

\section{Une mise en auvre effectuée au percuteur dur}

Les stigmates observés sur les nucléus et les enlèvements plaident en faveur de l'utilisation de percuteurs durs (86,6 \% des enlèvements). Aucun percuteur n'a été collecté sur le site.

Si les bulbes s'avèrent bien marqués, certains enlèvements présentent un esquillement du bulbe et de rares éclats sont affectés par une fracture de type Siret (5 pièces)

Cependant cette percussion semble «adoucie» sur environ $7 \%$ des pièces, ce qui pourrait témoigner de l'utilisation de pierre plus tendre ? Celles-ci illustrent toutes les phases de mises en œuvre de la matière première.

Enfin, quelques pièces (presque $6 \%$ des enlèvements) pourraient être issues d'un débitage pratiqué au percuteur tendre (?). Ces artefacts proviennent de toutes les séquences des chaînes opératoires, avec cependant une meilleure représentation des enlèvements issus du plein débitage $(57 \%)$.

\section{La préparation des plans de frappe}

Bien que de nombreuses pièces s'avèrent fragmentées (14\% de talons cassés), les plans de frappe non préparés semblent dominer (54\%) ; les talons préparés (dièdres et facettés) comptent pour $30 \%$ et les talons ôtés pour $1 \%$.

Cependant, les enlèvements à talons lisses sont souvent affectés d'une abrasion des corniches.

Au sein des artefacts dont le talon est reconnaissable, les enlèvements issus de séquences de décorticage (pièces présentant au moins la moitié de la face supérieure corticale) et d'aménagement de plan de frappe et de convexités (éclats laminaires affectés de cortex, lames ou lamelles corticales, lames à crête et initialisation) portent un talon majoritairement non préparé.

A l'inverse, les produits issus du plein débitage présentent plutôt des talons préparés : éclats laminaires et enlèvements Levallois (éclats et lames), à l'exception des lames à arêtes parallèles où la proportion entre talons préparés et non préparés s'équilibre.

Les produits issus des chaînes opératoires de débitage d'éclats (74,5\% des enlèvements)

Les éclats qui constituent l'assemblage lithique du niveau principal d'occupation comportent de nombreuses pièces liées au décorticage $(26,8 \%)$, à l'aménagement des surfaces de débitage et de préparation de plans de frappe $(13 \%)$. Les éclats de plein débitage représentent $40 \%$ des produits (fig. 24) et comportent des éclats Levallois ( $43 \%$ ), dont certains triangulaires et quelques enlèvements kombéwa (3\%).

La lecture des surfaces de plan de frappe et des talons témoigne d'un soin particulier accordé à la production prédéterminée contrairement à ce qu' on observe sur les éclats d'aménagement (37\% de talons préparés), les éclats indifférenciés issus d'une gestion de surface $(35 \%)$ et les enlèvements de décorticage (20\%).

La majorité des éclats de plein débitage a été obtenue au percuteur dur (plus de $89 \%$ ) et présente une abrasion des corniches $(75 \%)$.

Les éclats prédéterminés Levallois présentent majoritairement des talons préparés (facettés et dièdres : $66 \%$ ). Les corniches sont majoritairement abrasées $(83 \%)$.

\section{Les produits laminaires $(25,1 \%)$}

Les enlèvements laminaires comportent principalement des éclats d'aménagement de convexités latérales $(33,7 \%)$. Ceux-ci participent soit à l'aménagement des surfaces de débitage, soit à l'extension de la table laminaire des nucléus à débitage semi-tournant.

La lecture technologique des lames de plein débitage atteste d'une gestion de surface conduite depuis un plan de frappe, ou de deux plans de frappe opposés et d'un débitage tournant ou semi-tournant unipolaire et bipolaire opposé.

Les lames Levallois s'avèrent peu nombreuses $(2 \%)$, à la différence des lames de gestion unidirectionnelle (28\%) et des lames à arêtes parallèles (12\%) (fig. 25).

Ces dernières illustrent une gestion volumétrique de « type paléolithique supérieur ». «L'initialisation » du débitage peut se faire par l'utilisation d'un dièdre naturel (1 cas) ou par l'aménagement de crêtes à un versant ou à deux versants (4 pièces).

La majorité des lames de plein débitage a été obtenue au percuteur dur (plus de $77 \%$ ).

Comme dans le cas de la production d'éclats, la lecture des talons des lames atteste d'un soin particulier accordé à la préparation des surfaces de plan de frappe.

Si les talons ne sont pas systématiquement préparés (environ $47 \%$ de talons dièdres et facettés), l'abrasion des corniches s'avère majoritaire $(70 \%)$. 

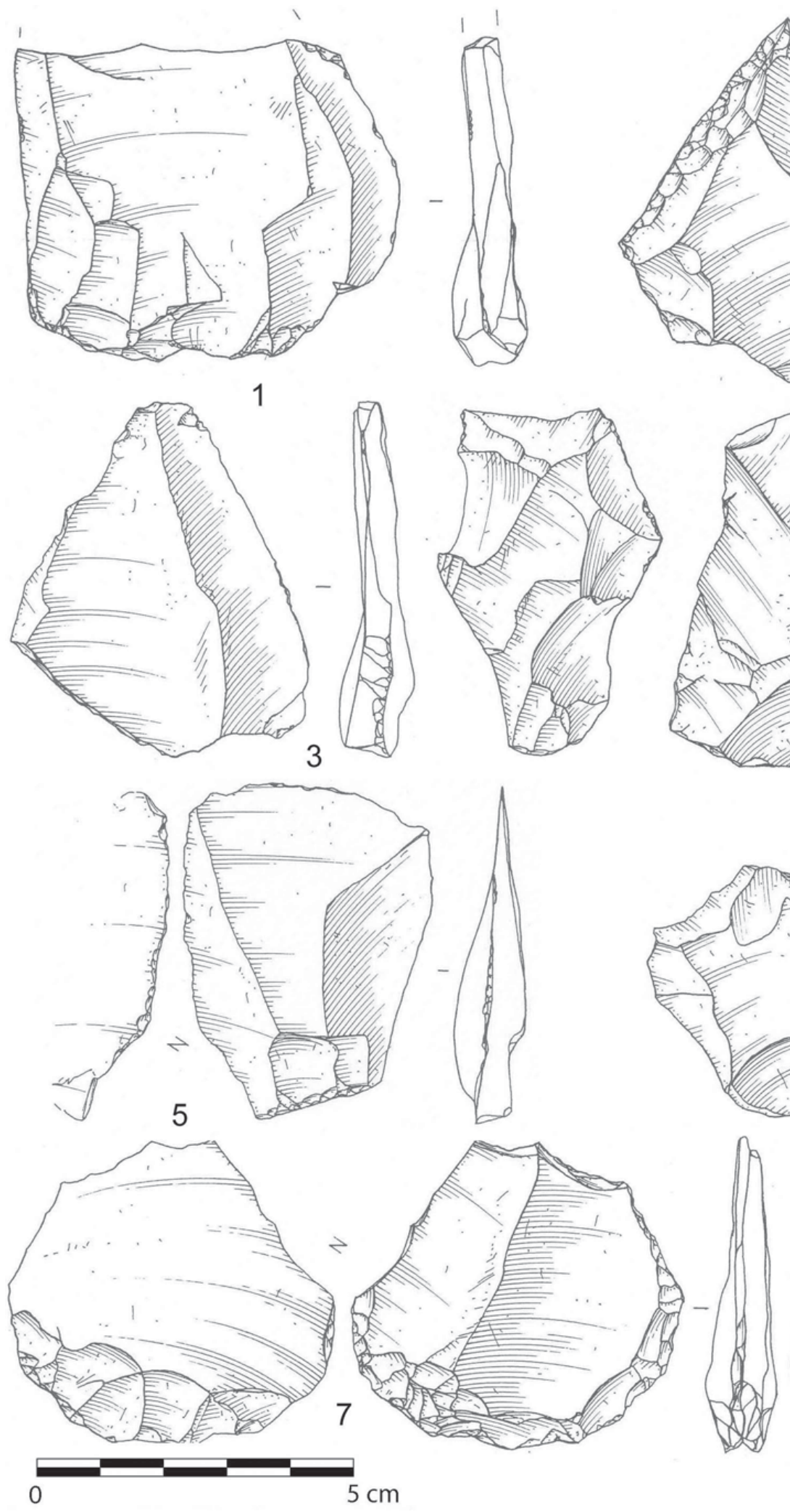
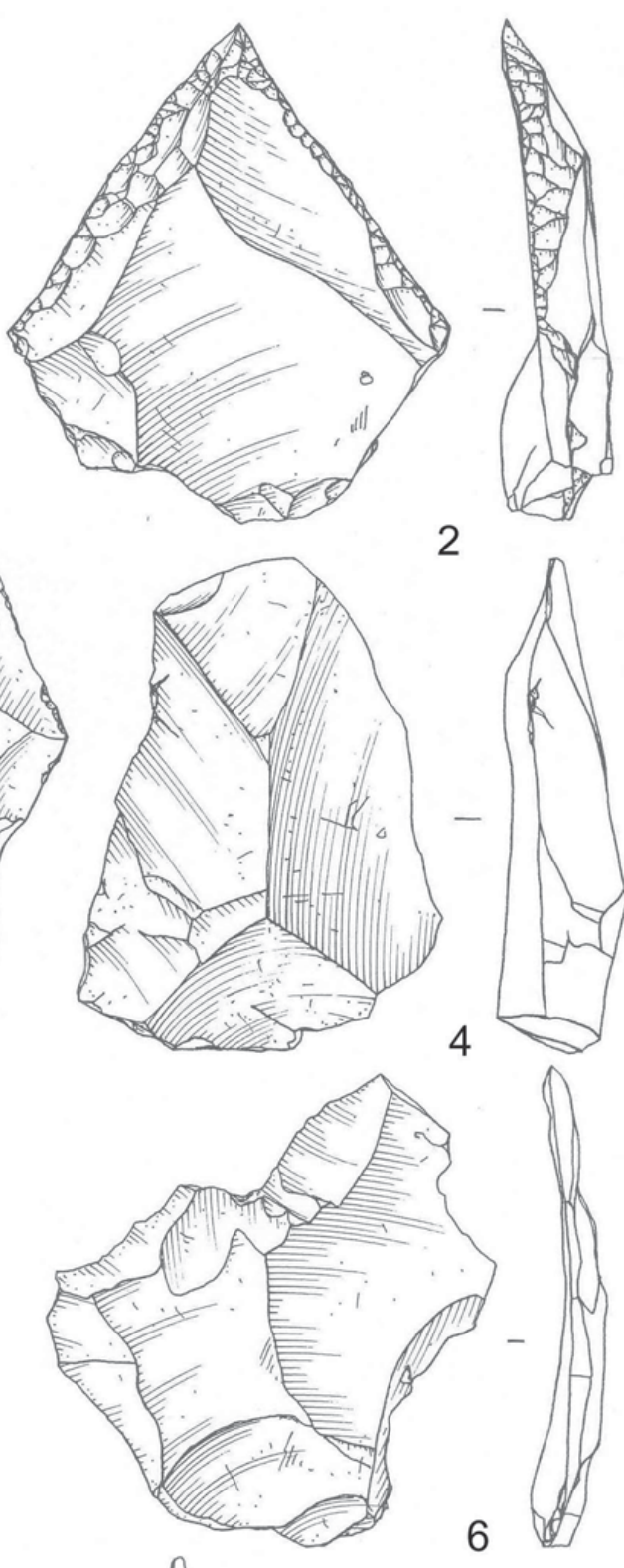

2

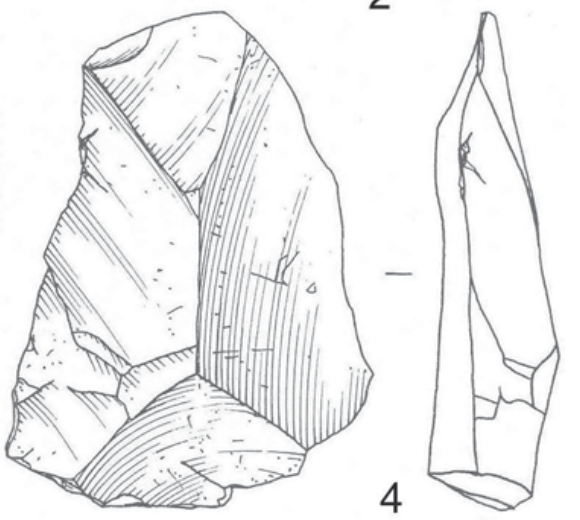

6

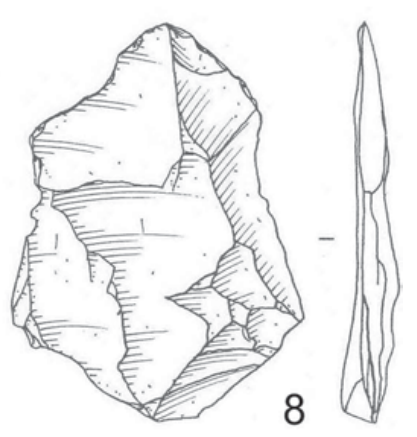

Fig. 24 : Grande Dune : Production d'éclats : Eclat Levallois : 1, Racloir double convergent ou pointe moustérienne sur éclat Levallois : 2, Eclat de gestion de surface : 3 ; Abri TR 67 : Eclats Levallois : 5, 6 et 8, Eclat de gestion de surface : 4, Eclat Levallois retouché avec amincissement du talon : 7 (Dessin P. Alix).

Fig. 24: Main Dune - flake production: Levallois flake: 1, double convergent scraper or Mousterian point on Levallois flake: 2, facial technology flake: 3; TR 67 shelter - Levallois flake: 5, 6 and 8, facial flake: 4, thinning Levallois flake: 7 (Drawing P. Alix). 
Les lames à arêtes parallèles sont pour moitié produites depuis un plan de frappe lisse. Ce fait s'observe également sur le mobilier issu du site proche de PortRacine à Saint-Germain-des-Vaux (Cliquet, 1994).

\section{5 - LES PIÈCES «TRANSFORMÉES »}

Dans cette catégorie figurent les enlèvements dont les tranchants sont modifiés par des retouches régulières, principalement des racloirs, des outils très spécifiques, tels les burins, et des artefacts affectés de coches dont l'origine anthropique n'est pas attestée. En effet les impacts liés au débitage et les processus taphonomiques, donc post-dépositionnels, sont susceptibles d'être à l'origine de la formation de coches et de denticulés.

Enfin, certaines pièces présentent des ébréchures continues irrégulières du fil du tranchant, qui pourraient être liées à l'utilisation (?).

Parmi ces dernières figurent un éclat kombéwa et un éclat d'aménagement.

Les enlèvements affectés de retouches marginales comptent :

- deux pièces à retouche abrupte mince concave modifiant le tranchant droit d'une lame à arêtes parallèles, dans sa partie mésiale, et un distal d'éclat Levallois ;

- une pièce à retouche abrupte mince convexe transformant le bord d'un éclat Levallois ;

- une retouche abrupte mince rectiligne alterne « aménageant » un éventuel éclat de façonnage.

Les encoches se rencontrent en position :

- proximale sur un éclat de décorticage et sur un éclat d'aménagement épais,

- latérale, retouchée sur un éclat Levallois

- en bout, sur un éclat de décorticage.

Un «denticulé » apparaît constitué d'un ensemble de coches directes et inverses sur la partie mésio-distale du support. Cet aménagement pourrait être lié à un emmanchement?

Les racloirs présentent majoritairement une retouche discrète. Il s'agit : d'un racloir latéral simple rectiligne aménagé sur une lame partiellement corticale (fig. 255 ) et de deux racloirs latéraux simples convexes confectionnés sur un éclat d'aménagement de convexité.

Un éclat Levallois présente un amincissement du bulbe et une ablation du talon (fig. 24-7). Cet aménagement «bifacial » a été obtenu par une retouche envahissante inverse, relativement plate et par une retouche directe oblique à sub-abrupte, prolongée par une retouche relativement marginale du type racloir latéral simple convexe.

Enfin, la série comporte, outre une chute de burin, un burin d'angle sur lame (fig. 25-9 et 13). Le dièdre a été obtenu par l'intersection d'un petit éclat transversal à l'axe morphologique de la pièce, affecté de deux enlèvements laminaires.

\section{6 - CARACTÉRISTIQUES ET SIGNIFICATION TYPO-TECHNOLOGIQUE DE LA SÉRIE}

L'assemblage lithique du Rozel résulte de la mise en œuvre de galets marins de petites dimensions et de blocs de quartz de filon, présents dans l'habitat. Cette composante mixte confère une originalité toute particulière à cette série. En effet, l'utilisation du silex apparaît quasi exclusive dans les sites du Cotentin; seuls quelques rares artefacts témoignent de la mise en œuvre de quartzite, de quartz, de grès et de granite. Comme pour le silex, ces matières premières sont prélevées dans les cordons littoraux.

Le recours à une roche de substitution traduit le manque de silex, notamment dans les cordons littoraux, vraisemblablement masqués par les massifs dunaires.

Ce comportement n'est pas sans rappeler les nombreux sites du Massif Armoricain breton, où les roches de substitution au silex occupent une place importante (Cliquet \& Monnier, 1993).

La lecture technologique du mobilier atteste du rôle prépondérant joué par la nature de la matière première sur les processus d'exploitation.

Les schémas de débitage mis en œuvre sur les blocs parallélépipédiques de quartz s'avèrent peu élaborés et de ce fait peu productifs. Le tailleur exploite les surfaces naturelles des blocs sans aménagement des convexités et sans préparation des surfaces de plan de frappe. La production s'achève rapidement par rebroussement des enlèvements et «fermeture » des angles de débitage. Les produits ne sont pas standardisés.

La mise en œuvre des galets de silex apparaît plus diversifiée, avec notamment des chaînes opératoires à éclats et à lames.

\section{La production d'éclats}

Au sein de la chaîne opératoire à éclats, plusieurs schémas de débitage sont attestés par la lecture technologique des artefacts (nucléus et enlèvements).

Le débitage d'éclats est régi selon des schémas non Levallois et Levallois.

A l'exception d'un galet affecté par le gel, dont l'exploitation n'est pas poursuivie, et d'un fragment de nucléus qui témoignerait d'un débitage sécant, la production d'éclats est conduite selon un débitage parallèle Levallois.

Les modalités d'exploitation s'effectuent selon un schéma variable: bipolaire et bipolaire orthogonal, parfois alternatif, et centripète.

L'assemblage lithique du Rozel se caractérise par une production originale de micro éclats prédéterminés. Ce phénomène d'élaboration de «microlithes Levallois » a déjà été observé sur le site de Grossœuvre dans le département de l'Eure (Cliquet \& Lautridou, 2000).

Au Rozel, comme à Grossœuvre, ce « microlithisme prédéterminé» est illustré par des nucléus et des 

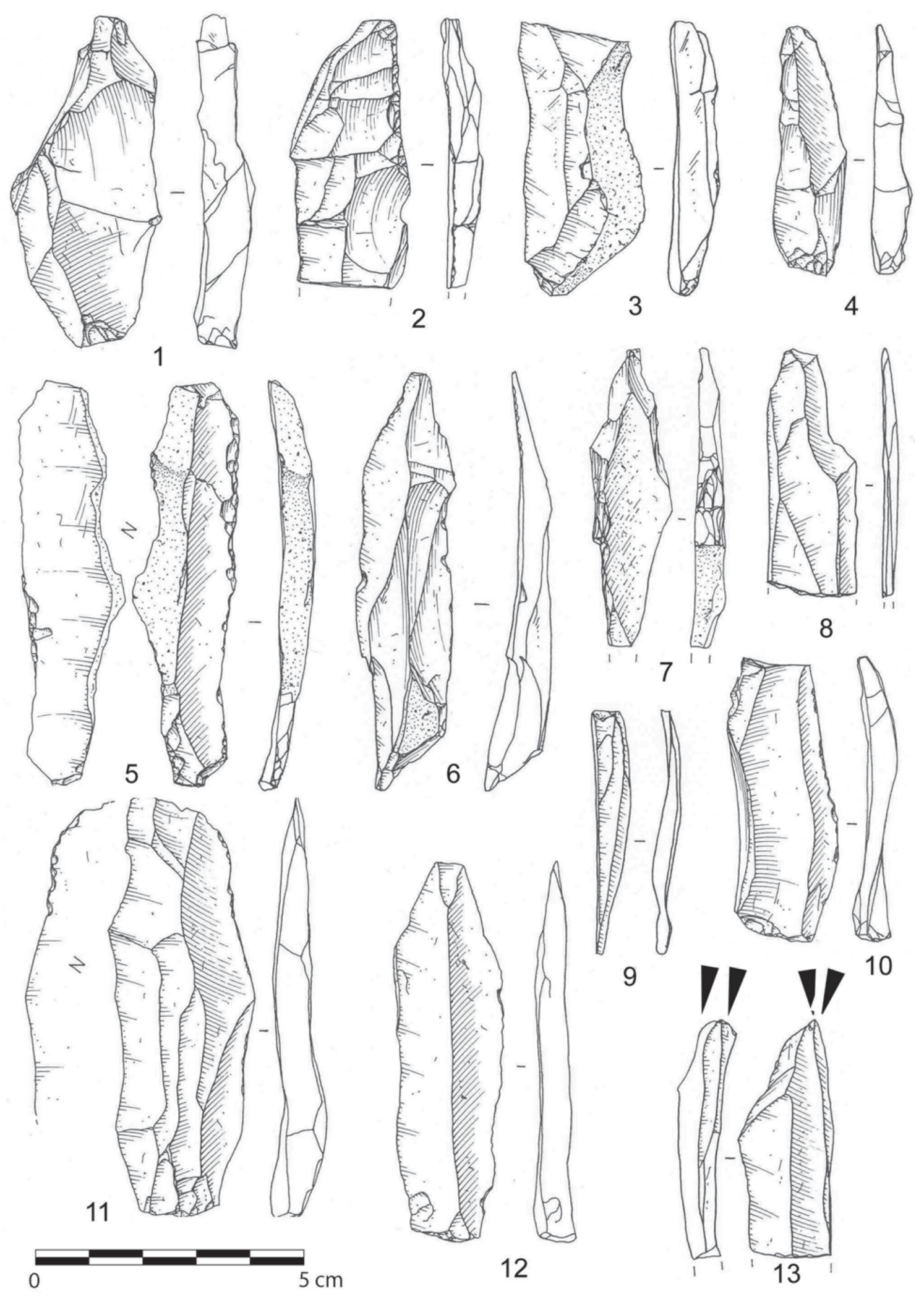

8
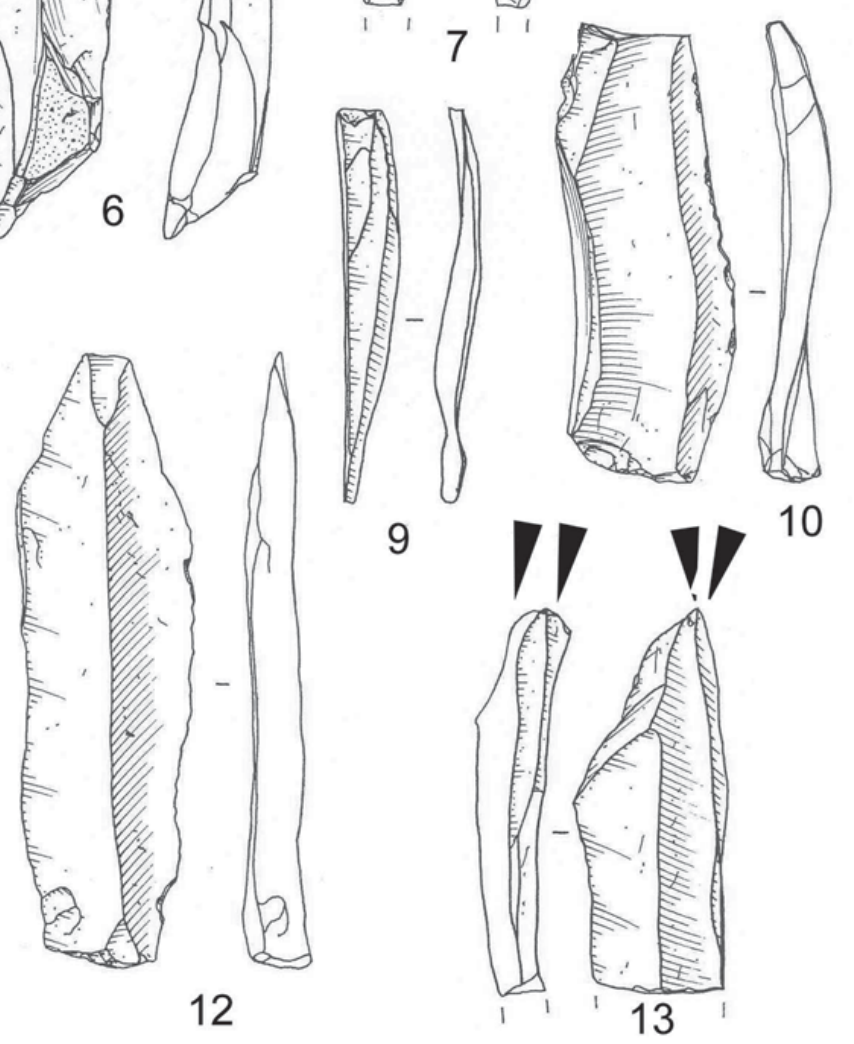

12

Fig. 25 : Abri TR 67 - Production laminaire : Eclats laminaires et lame aménageant une convexité : 1, 3 et 5 , Lame à crête latérale : 2, Lame à crête seconde : 4 , Lames de plein débitage $: 6,8,10,11$ et 12 , Lame à crête partielle : 7, Lamelle ou chute de burin (?) : 9 et $13:$ Burin sur lame (Dessin P. Alix).

Fig. 25: TR 67 shelter - blade production : bladed flakes: 1, 3 and 5, blade with a lateral crest: 2, blade with a secondary crest: 4, blade in full splinting: 6,8,10,11 and 12, partially crested blade: 7, little blade or 'chute de burin' (?): 9 and 'burin' on blade: (drawing P. Alix). 
enlèvements de très petites dimensions (respectivement inférieurs à $50 \mathrm{~mm}$ et à $15 \mathrm{~mm}$ ), et par des raccords et des remontages de produits sur les nucléus (fig. 23-1 à 4).

La nature de la matière première témoigne d'une sélection des nodules à structure cristalline fine et homogène. A Grossœuvre, ce sont des matières premières exogènes qui ont été exploitées.

\section{La production de lames}

Cette production est conduite selon un schéma semi-tournant unipolaire produisant des lames à arêtes parallèles. L'initialisation du débitage peut se faire soit par un dièdre naturel, soit par une crête à deux versants.

Deux types de gestion seraient présents, une gestion par débitage direct utilisant les convexités naturelles du rognon (lame à dièdre naturel, lame à « dos cortical » qui témoigneraient de l'initialisation et de l'extension de la table laminaire), et une gestion de « type paléolithique supérieur » attestée par la présence de lames à crête à un seul versant, à deux versants et des lames à « dos cortical».

Si les nucléus retrouvés illustrent un débitage conduit depuis un unique plan de frappe, quelques lames à arêtes parallèles témoignent d'un débitage bipolaire.

\section{Finalité de la production lithique}

La série comporte peu de pièces retouchées. Ce sont de rares racloirs à retouche marginale, quelques pièces à coches et affectées de retouches peu marquées.

Parmi les enlèvements " transformés en outil », il ne semble pas y avoir de sélection des supports d'outils, à l'exception du burin aménagé sur lame (?).

\section{7 - LE ROZEL DANS LE COMPLEXE LAMINAIRE DU PALÉOLITHIQUE MOYEN RÉCENT (D.C.)}

L'assemblage lithique du Rozel présente donc de grandes affinités avec le mobilier du site de PortRacine à Saint-Germain-des-Vaux (Cliquet, 1994) :

- débitage d'éclats, notamment selon des schémas Levallois,

- production de lames par débitage semi-tournant direct et de «type paléolithique supérieur »

- discrétion des supports transformés en outil.

Cependant à Port-Racine, la production d'éclats s'avère plus diversifiée, notamment le débitage direct exploitant les convexités naturelles des nodules, et seul le silex issu des cordons littoraux a été mis en œuvre.

Comme l'ont déjà souligné S. Révillion et $\mathrm{A}$. Tuffreau (1994), la coexistence de chaînes opératoires réservées à la production d'éclats et de lames est un phénomène étendu géographiquement (spatialement) et dans le temps (chronologiquement), durant tout le stade 5 .
L'intégration du site du Rozel au groupe des industries laminaires de la phase récente du Paléolithique moyen avait été pressentie (Cliquet, 1994).

\section{Fonction du site}

L'analyse taphonomique des vestiges témoigne d'une occupation de pied de falaise, initialement peu dilatée, et déstructurée par des phénomènes géologiques, qui rappelle les implantations des sites 1 et 5 de Port-Racine, à Saint-Germain-des-Vaux, et de la Mondrée à Fermanville.

Les abris de Port-Racine et de la Mondrée sont exposés plein est, le Rozel s'ouvre au sud. La position du platier et la géomorphologie globale du site (fig. 2) attestent que ce site n'a pas pu être occupé en période de haut niveau marin (falaises et abris balayés par les marées). D'une manière générale, l'accès via l'intérieur des terres a dû aussi être très limité par le caractère dense de la forêt interglaciaire. La structuration de l'espace s'organise comme en abri sous roche depuis le pied de falaise, (espace semi ouvert) et comporte des structures de combustion (au Rozel et à Port-Racine) et des amas de débitage (à Port-Racine et à la Mondrée).

L'abri du Rozel pourrait correspondre à un habitat saisonnier, peut-être aussi occupé par des carnivores ? La publication et les rapports de fouilles attestent de la découverte de deux coprolithes dont "l'état "écrasé" de ces restes et le broiement de leur contenu ne permettait aucune analyse utile » (Scuvée \& Vérague, 1984) et « d'amas, véritables magmas d'esquilles et de fragments ramollis » (rapport 1969, p. 7). Ces éléments ne sont pas sans évoquer les déjections de carnassiers, notamment les fèces de loups, incorporant de nombreux petits débris d'os et esquilles d'os longs (Binford, 1981 et 1988 in Rigaud et al.). Cependant, comme le souligne B. Van Vliet-Lanoë, le développement de la podzolisation au niveau D2c2 a permis, en extérieur, le « ramollissement » avec préservation du collagène des petits fragments osseux probablement déjà attaqués par le gel et les algues, comme nous pouvons l'observer actuellement au Spitzberg. Une évolution analogue des os a été observée également dans le site de la Cotte de Saint-Brelade à Jersey (Van Vliet-Lanoë in Callow \& Cornford, 1986). Il faut enfin rappeler qu'aucune marque indiquant l'action d'un quelconque carnivore n'a été observée sur les ossements. De fait, l'abri du Rozel semble bien avoir été occupé uniquement par les Hommes (cf. ci-dessus Patrick Auguste).

\section{8 - SYNTHÈSE PALÉOENVIRONNEMENTALE ET CHRONOLOGIQUE (B. V. V.-L.)}

Quatre phases majeures composent cette séquence: le complexe littoral eemien associé au premier niveau d'occupation humaine (E2), un premier interstade tempéré, associé au second niveau d'implantation anthropique, un second interstade tempéré et enfin le Pléniglaciaire (fig. 8, 28 et 29). Afin de documenter 
l'évolution environnementale du site, nous avons recherché des analogues actuels à différentes latitudes.

\section{Le complexe littoral éémien}

A la base, D0 correspond à un bas d'estran à $+4 \mathrm{~m}$ NGF, le maximum eustatique de l'Eemien (OIS 5e-a) communément daté entre 130 et $125 \mathrm{ka}$. Le niveau maximum (haute mer, $\delta^{18} \mathrm{O}$ ) de l'Eemien est généralement localisé vers 4-6 m (Overpeck et al., 2006), audessus du niveau marin actuel (+ 5 m NGF). Pour le Rozel cette correspondance donnerait au moins $+10 \mathrm{~m}$. NGF pour les hautes mers (actuellement $9 \mathrm{~m}$. de marnage), sans tenir compte des surcotes de tempêtes. Lors de ce maximum, la paléofalaise a dû être érodée et déblayée des dépôts de pentes du Saalien (OIS 6). Une accumulation de blocs et galets de haut niveau a pu encombrer les abris sous-roches, régulièrement balayés par les tempêtes (excavation de l'abri).

D1a1 correspond à un dallage d'origine glacielle de bas d'estran, riche en éléments de schistes. Ensuite le niveau marin a régressé sous le niveau actuel en association avec une dynamique initialement glacielle, se poursuivant par le dépôt du head sableux et humifère D1a2. Elle est également bien visible à Port-Racine et à la Cormorandière (fig. 27-d ; Van Vliet-Lanoë, 1988 ; Hallégouët \& Van Vliet-Lanoë, 1989). Au Rozel, le niveau marin remonte ensuite vers $+4 \mathrm{~m}$ NGF lors de l'épisode D1b (estran moyen ; OIS $5 \mathrm{e}_{\mathrm{c}}$, fig. 28) ce qui est légèrement en contrebas du niveau marin moyen actuel.

D1c (sable compacté par le swash de tempête, $+5 \mathrm{~m}$. NGF) et daté à $115 \pm 11$ ka correspondrait donc à un second maximum eustatique de l'Eemien, associé à une dérive littorale du sud-ouest (grès et silex), presque immédiatement suivi par une régression. Il n'y a pas d'évidence de dynamique glacielle en D1c, mais bien de ruissellement (ravines) et un cordon abandonné (D1c). La dune carbonatée (loupe remaniée au sein de D3, datée à $113 \pm 11 \mathrm{ka}$ ) se met en place à cette époque. Une situation analogue existe actuellement au sud des Mielles de Biville (fig. 26) : un grand corps dunaire correspondant aux refroidissements de l'époque mérovingienne et du Petit Age Glaciaire repose sur un cordon datable de la période gallo-romaine et « escalade » actuellement la paléofalaise en contexte encore tempéré (Meurisse et al., en préparation). La malacofaune préservée est conforme à cette situation tempérée et au caractère initialement carbonaté des sables dunaires. Le sable envahit les abris sous-roches du Rozel, habités plus ou moins temporairement (couche E2), en fossilisant les ossements et en les préservant. En dehors de l'abri, le sable est probablement remanié en plusieurs épisodes avec des dépôts de pente comme en atteste l'analyse au MEB, puis illuvié par une pédogénèse. La datation situerait l'évènement au début du OIS5d (D1d : $107 \pm 10 \mathrm{ka})$.

\section{La dynamique glacielle}

Au Rozel, D1c repose sur un platier à $+4 \mathrm{~m}$. NGF ou sur un estran (D1a-b) avec des caractéristiques glacielles particulièrement bien préservées sous la «Petite Dune ». Les estrans glaciels sont caractérisés (fig. 27-e) par une surface basale horizontale tronquant aussi bien les sédiments meubles que la roche, souvent gélifractée et portant des marques d'arrachage et un dallage de blocs (fig. 27-a et b ; Dionne, 1998 ; Van Vliet-Lanoë, 2005). La matrice fine de l'estran est souvent vaseuse ou très enrichie en gélifracts, ce qui est le cas ici.

Cette dynamique glacielle s'accorderait avec la présence d'un os de morse, associé au premier niveau d'occupation (E2) signalé par P. Auguste. Cet animal fréquente le plus souvent la banquise littorale sur fonds peu profonds riches en coquillages dans des endroits, où l'eau ne gèle pas durant l'hiver, tels que polynies. Les morses préfèrent se retrouver sur la glace ; cependant l'été, ils vont sur les rives et sur les plages lorsque la glace se fait rare. Une population de morses du nordouest de l'Atlantique qui élevait ses petits dans le Golfe du Saint-Laurent $\left(+6^{\circ} \mathrm{C}\right.$ TMA) a actuellement disparu ; elle illustre cependant des conditions environnementales similaires à celles rencontrées au Rozel à l'époque : grands platiers littoraux comme ceux qui longent l'ensemble de la péninsule du Cotentin.

Ensuite la topographie se stabilise avec un haut niveau marin, en contre-bas (vers $-25 \mathrm{~m}$; Overpeck et al., 2006), une large plaine herbeuse et probablement arborée s'étend au sud-ouest du site, la falaise étant boisée avec des espèces thermophiles (feuillu, if ; fig. 12). Une faune tempérée colonise la plaine. Un sol lessivé se forme au début du OIS 5c - Saint-Germain I, sur ce qui subsiste des dunes carbonatées attestant une situation analogue à celle des sables dunaires remaniés de Belcroute et de Portelet (Jersey, Keen et al., 1996). Ce sol est tronqué par des dépôts de pente à dynamique de cryoreptation, ce qui dénote un contexte déjà très froid en hiver. Les hivers deviennent plus rudes, avec de fortes chutes de neige, induisant une acidification marquée du site comme dans le cas de Port-Racine et d'Ecalgrain (Van Vliet-Lanoë, 1988 ; Cliquet, 1994). Ce refroidissement vers 101 ka est corrélé, selon Shackleton et al. (2003), avec Montaigu reconnu à la Grande Pile par Woillard (in de Beaulieu \& Reille, 1992). Il correspond vraisemblablement au refroidissement post-Amersfoort observé par Loyer et al. (1995). Ensuite la pédogenèse reprend sous la forme de sol podzolique, correspondant à l'optimum thermique du OIS 5c (fig. 8). Une occupation répétée des abris s'établit vraisemblablement entre 98 et $95 \mathrm{ka}$, probablement protégée du sud par un cône de débris. Cette occupation pourrait être synchrone avec le site analogue de Seclin (91-95 \pm 11 ka ; Révillon \& Tuffreau, 1994). La proximité de paléocordons de galets à l'affleurement dans cette plaine devait fournir du silex amené par la dérive littorale glacielle (arrachage ; Hallégouët \& Van Vliet-Lanoë, 1989).

Après, le climat se dégrade rapidement (D3a1) et la masse dunaire s'effondre en masses successives chargées de blocs (fig. 8 et 28). Lors de la partie la plus froide du OIS $5 b$, les vestiges des accumulations 

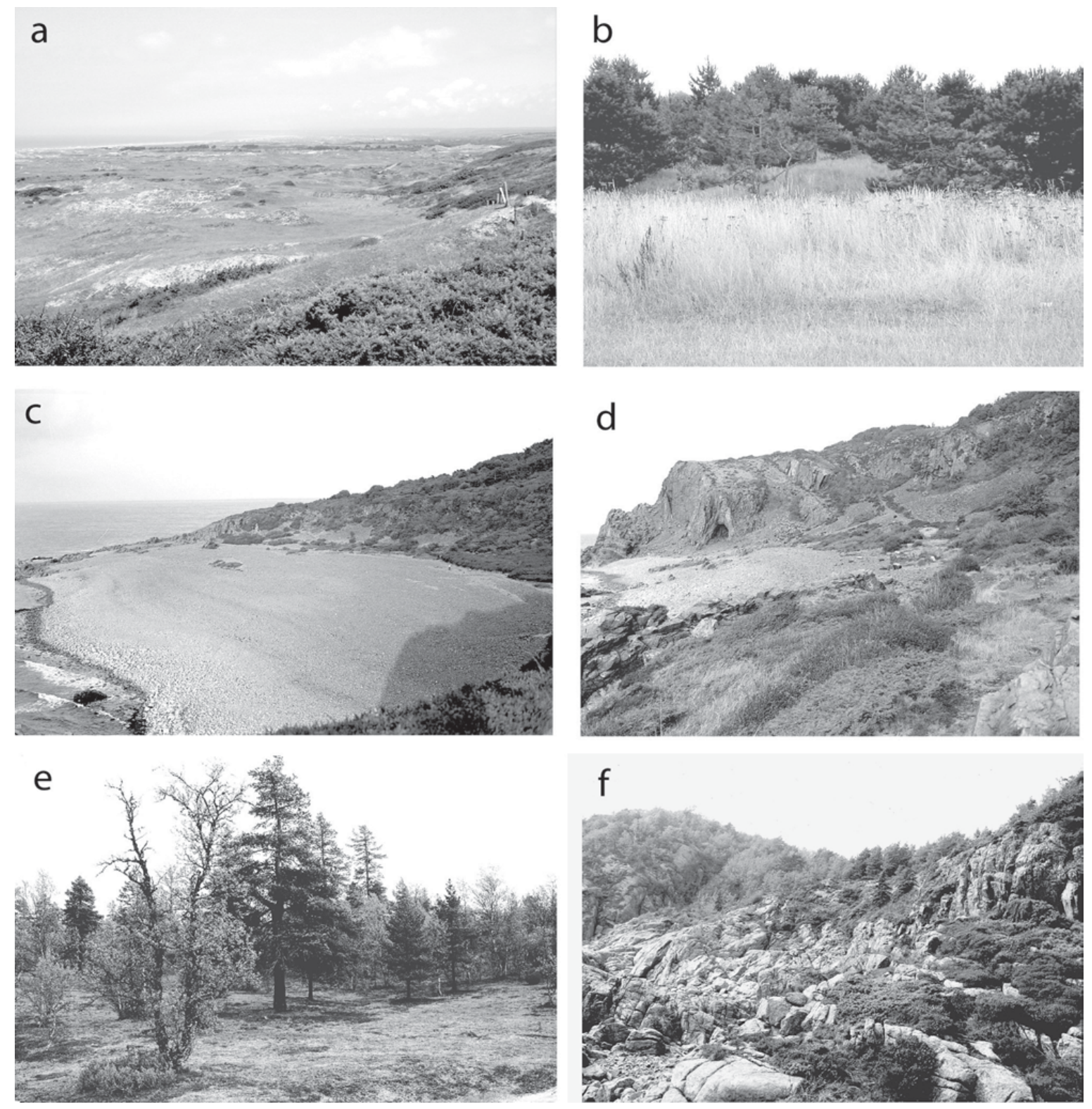

Fig. 26 : a-b) Les Mielles de Biville (Manche) : évocation de l'environnement du Rozel à la fin du stade 5e.; c-d) contexte boréal, avec plages abandonnées, comme au Rozel et à Port-Racine (Hovshallard, Sud de la Suède); e) forêt boréale herbacée "sèche " sur sable dunaire (Finlande) ; f) littoral rocheux boréal de la région de Kristiansand (Sud de la Norvège) (clichés B. Van Vliet-Lanoë).

Fig. 26: $a-b)$ The Mielles dune field at Biville (Manche): the Rozel at the end of OIS $5 e ; c-d$ ) boreal situation with raised beaches, like at the Rozel and at Port Racine (Hovshallard, southern Sweden); e) dry boreal forest on dune sand (Finland); rocky coast from Kristiansand (southern Norway) (pictures B. Van Vliet-Lanoë).

dunaires sont remaniés, avec des chutes de blocs en pied de falaise et une charge de plus en plus soutenue en gélifracts. Il est associé à une pénétration importante du gel et à de la solifluxion (Van Vliet-Lanoë, 1986, 1988) qui affecte déjà D2C2 au Rozel. A cette époque, vers 90 ka le site est probablement abandonné, la lande boréale recouvrant le site devenant probablement moins riche : il devrait y avoir normalement du renne et de l'orignal, absents du cortège faunique recensé. Le ruissellement dans les abris et en pied de versant devait rendre le site peu praticable en été, notamment en raison de la prolifération des moustiques (fig. 28). La situation écologique devait être analogue à celle qui existe actuellement dans les champs de dunes continentales du nord de la Finlande. Ensuite, le contenu des abris est sapé par les eaux de fonte de neige au printemps (suffosion) et bascule vers le fond vidant les abris. Une autre partie s'effondre vers l'extérieur suite à l'affaissement par solifluxion du pied de dune, amenant leur remaniement sous la forme des strates définies par Scuvée (D6 et E3). L'effondrement de la dune commence à affecter superficiellement le massif D1c. 

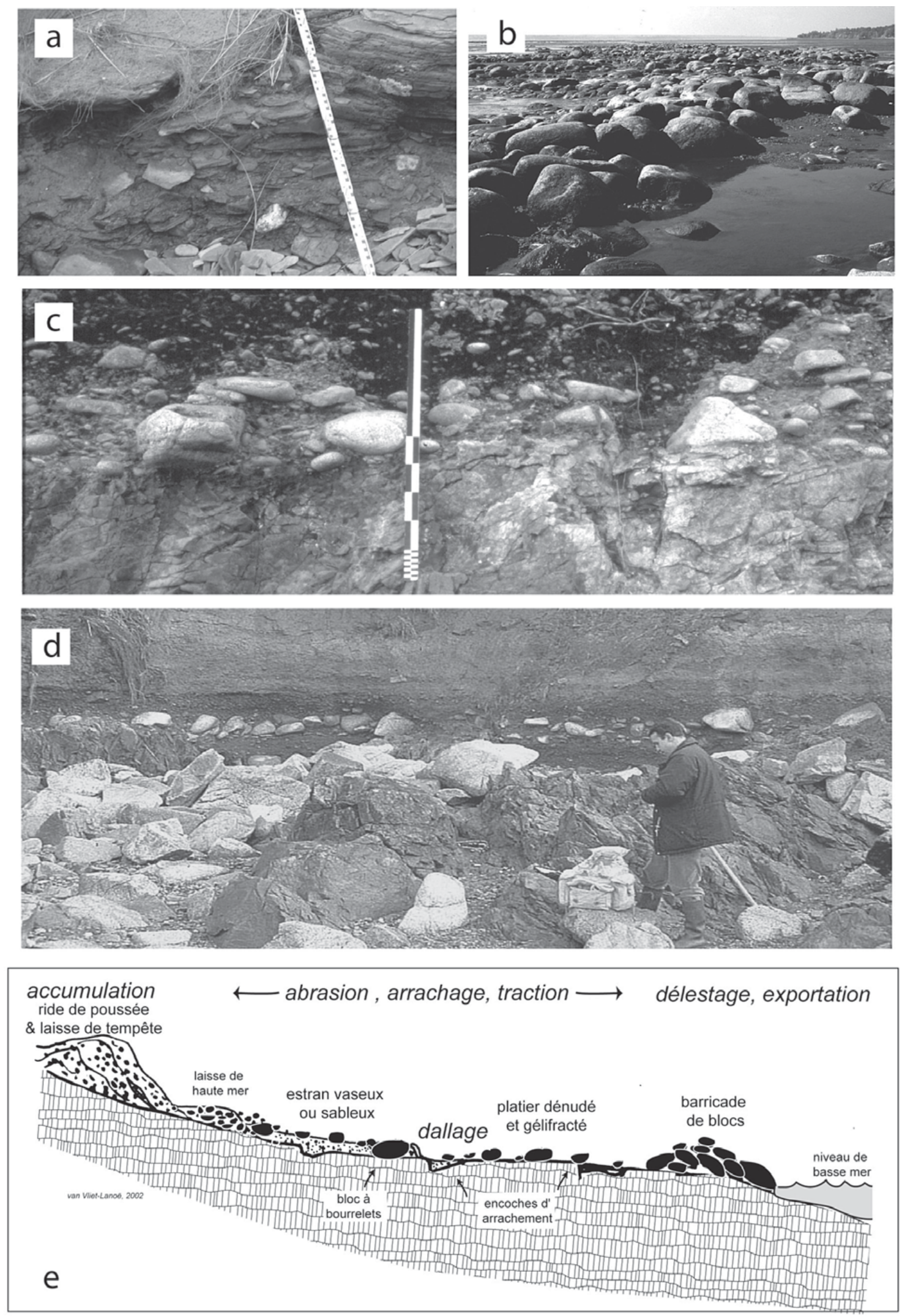

Fig. 27 : (a) Plage fossile à blocs glaciels, unité D1, sous TR 67 ; (b) plage à blocs glaciels, St Laurent, Cap Rouge ( Québec) ; (c et d) plage fossile à blocs glaciels, Mid Eemian Cooling, (c) Port-Racine, (d) La Cormorandière; (e) schéma d'un estran glaciel (d'après Van Vliet-Lanoë, 2005). Fig. 27: a) Beach with shore ice rafting, OIS 5e, Le Rozel; c) beach with shore ice rafting, OIS 5e, Port Racine; d) beach with shore ice rafting, OIS $5 e$, La Cormorandière; e) sketch of functionning of a beach with shore ice rafting (Van Vliet-Lanoë, 2005). 
a
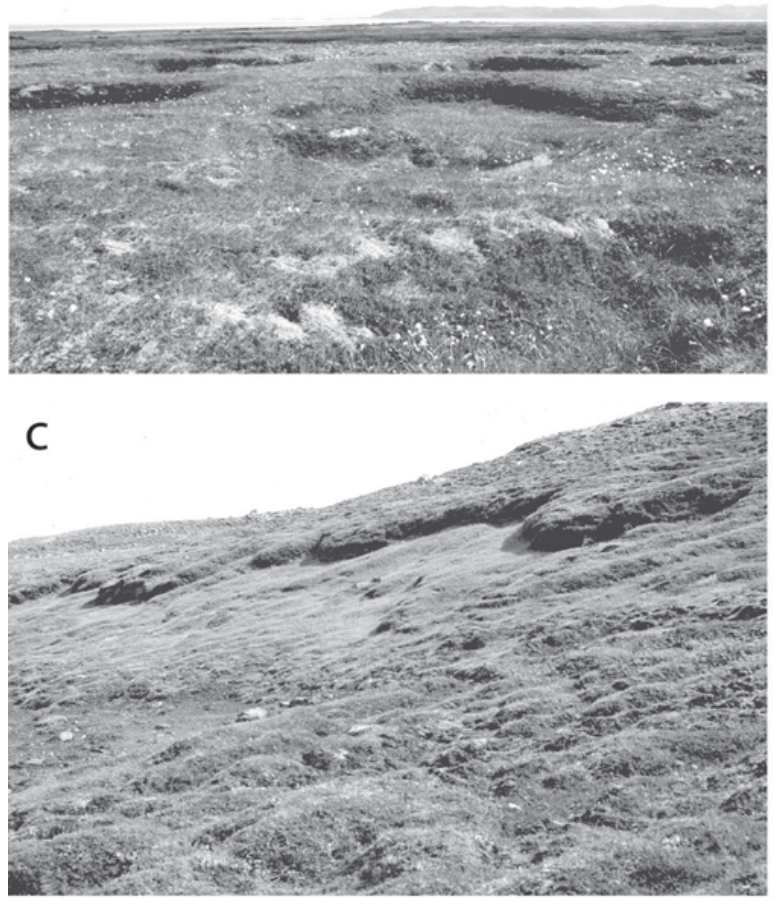

e

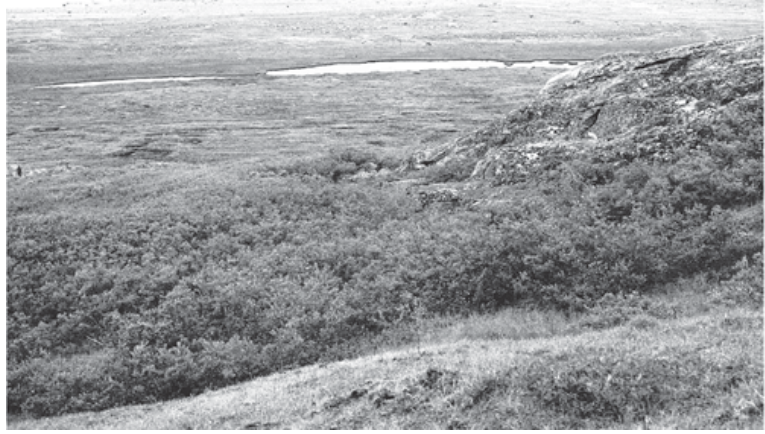

b
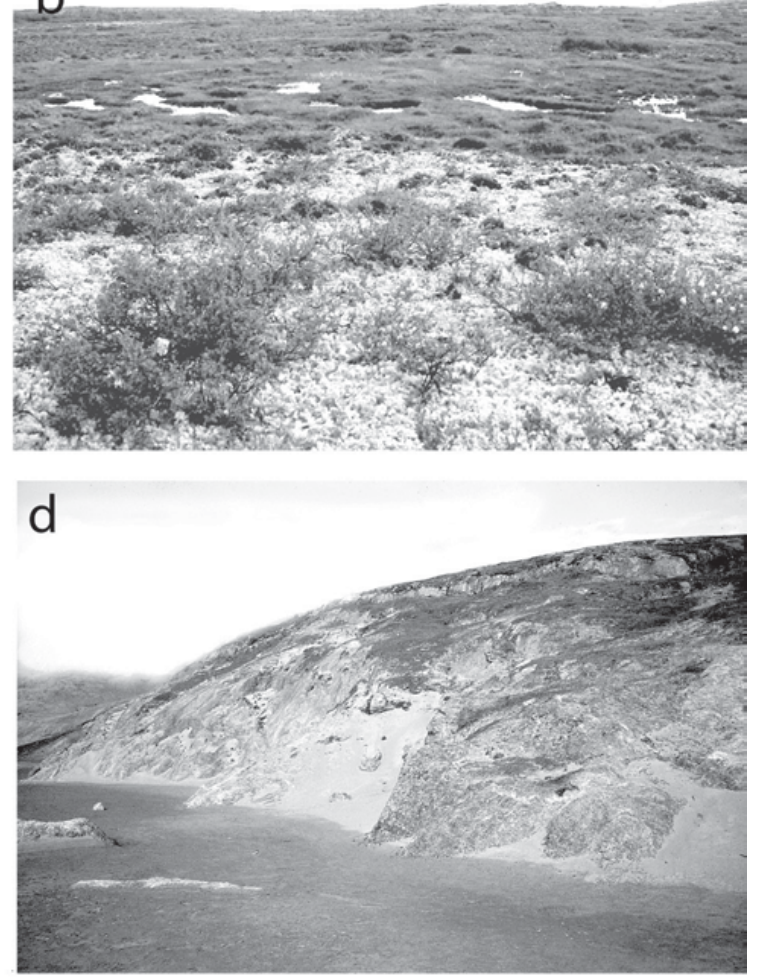

$\mathrm{f}$

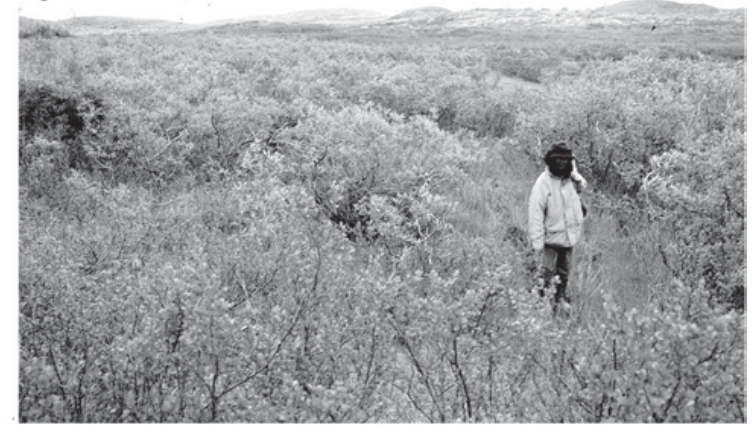

Fig. 28 : Le Rozel subarctique. a-b) Végétation océanique subarctique tourbeuse (avec moustiques ! Vesteralen Norvège) sur zone sableuse podzolisée) ; c) coulées de solifluxion ( $\mathrm{N}$ de l'Islande); d) placage éolien sur côte rocheuse exondée (Spitzberg); e) toundra buissonnante préservée par une congère sur versants exposés au Nord (Ungava); f) zone humide basse à saules (Nord Québec) (clichés B. Van Vliet-Lanoë).

Fig. 28: Arctic Rozel site. a-b) peaty subarctic oceanic vegetation on podsilic sands (with mosquitos! Vesterálen, Norway) ; c) solifluction lobes (N.Iceland) ; d) eolian fixation on a bare rocky coast (Svalbard); e) shrub tundra preserved by snow bank on a north facing slope (Ungava) ; $f$ ) humid willow shrub tundra (Northern Quebec) (photos B. Van Vliet-Lanoë).

Le site est abandonné définitivement. Ce refroidissement brutal et marqué de la fin du OIS 5c est daté à 87 ka par Shackleton et al. (2003).

Après cette période d'instabilité marquée, le climat se radoucit quelque temps et un nouveau sol lessivé, assez rubéfié se développe au sommet de D3, comme dans le site de Nantois après $83 \pm 13$ ka (Baie de SaintBrieuc ; Loyer et al., 1995) ou à Belcroute (Jersey, Keen et al., 1996). Il correspond au OIS 5a ou Odderade - Saint-Germain II, entre 82 et 75 ka selon Shackleton et al. (2003). Le contexte est probablement assez voisin de celui de l'interstade précédent, avec une steppisation encore plus marquée et un boisement peu dense en conifères (Van Vliet-Lanoë, 1986, 1988).
L'habitat est enfoui sous les dépôts de pente ; il ne sera mis au jour que par l'érosion très récente du site.

Ensuite, les dépôts de pente deviennent dominants à partir du OIS 4 (<75 ka selon Shackleton et al., 2003) jusqu'à la fin du dernier Glaciaire, avec quelques épisodes (E2) permettant, comme à Ecalgrain et à Port Racine, le développement de petits sols de prairie subarctiques (Van Vliet-Lanoë, 1988). La datation obtenue à Nantois $(67 \pm 10 \mathrm{ka}$, Loyer et al., 1995) est cohérente pour cet ensemble.

L'enregistrement des transgressions éémiennes dans le site du Rozel pourrait enfin concilier les différences reconnues entre l'Eemien terrestre (ex. de Beaulieu \& Reille, 1992; Sanchez-Goni et al., 1999) contraint 
entre 130 et $107 \mathrm{ka}$ et, le stade isotopique 5e marin (OIS 5e) contraint entre 135 et $120 \mathrm{ka}$ (Shackleton et al., 2003). La période comprise entre 120 et $107 \mathrm{ka}$ (le début du refroidissement marqué du OIS 5d) se matérialiserait par une remontée du niveau marin à une cote très voisine de l'actuelle vers $115 \mathrm{ka}$, une faune tempérée au Rozel et, par une flore locale attestant de conditions toujours tempérées (charbons de bois de feuillus) à cette époque dans le site analogue de Port Racine, (niveau de base; Cliquet, 1994), alors que l'océan Atlantique du nord-ouest s'est déjà refroidi (Cortijo et al., 1994 ; Frontval \& Jansen, 1997 ; Adkins et al. 1997). Ceci confirmerait les observations de Mac Manus et al. (1994) : l'Atlantique du nord-est reste tempéré jusqu'au début du OIS 5d (zone C24) sous l'influence d'un Gulf Stream fonctionnel. Dans les coupes préservées, il n'y a pas d'évidence de banquise contemporaines de la seconde régression $(107 \mathrm{ka})$.

\section{9 - CONCLUSION GÉNÉRALE}

L'accès aux minutes de fouilles, à l'industrie lithique et à la quasi-totalité du mobilier osseux collecté par l'inventeur du gisement (Y.R.), les analyses des données anciennes et les nouvelles investigations menées sur le site nous ont permis de revisiter le site du Rozel tant au plan chronostratigraphique qu' au plan culturel.
Contrairement aux conclusions livrées dans la monographie publiée par F. Scuvée et J. Verague (1984), qui attribuaient l'occupation de l'abri du Rozel au Châtelperronien, il est maintenant établi, sur base stratigraphique et à partir d'un ensemble de datations absolues (OSL) que la position chronostratigraphique de l'industrie se place en début du Dernier Glaciaire (vers $115 \mathrm{ka})$.

L'occupation initiale du site (niveau E2 de Scuvée \& Vérague, 1984) pourrait être contemporaine de l'implantation inférieure du secteur 4 du gisement de Port Racine, en association avec un épisode de refroidissement du climat et de régression marine connu comme le «Mid Eemian Cooling ». La position du platier et la géomorphologie globale du site (fig. 2) attestent que ce site n'a pas pu être occupé en période de haut niveau marin (falaises et abris balayés par les marées). D'une manière générale, l'accès au littoral par l'intérieur des terres a dû rester difficile étant donné le caractère dense de la forêt interglaciaire. Le site n'a pu être accessible que lorsque la régression du Mid Eemian Cooling a abandonné un estran sableux contournant une large part de la côte du Cotentin, émergeant de surcrô̂t le seuil du Cotentin.

Ces éléments réfutent donc l'attribution de cette industrie laminaire au Paléolithique supérieur. L'analyse fine de la stratigraphie et les données paléoécologiques, notamment malacologiques, permettent d'attribuer les niveaux d'occupation du site à la fin de

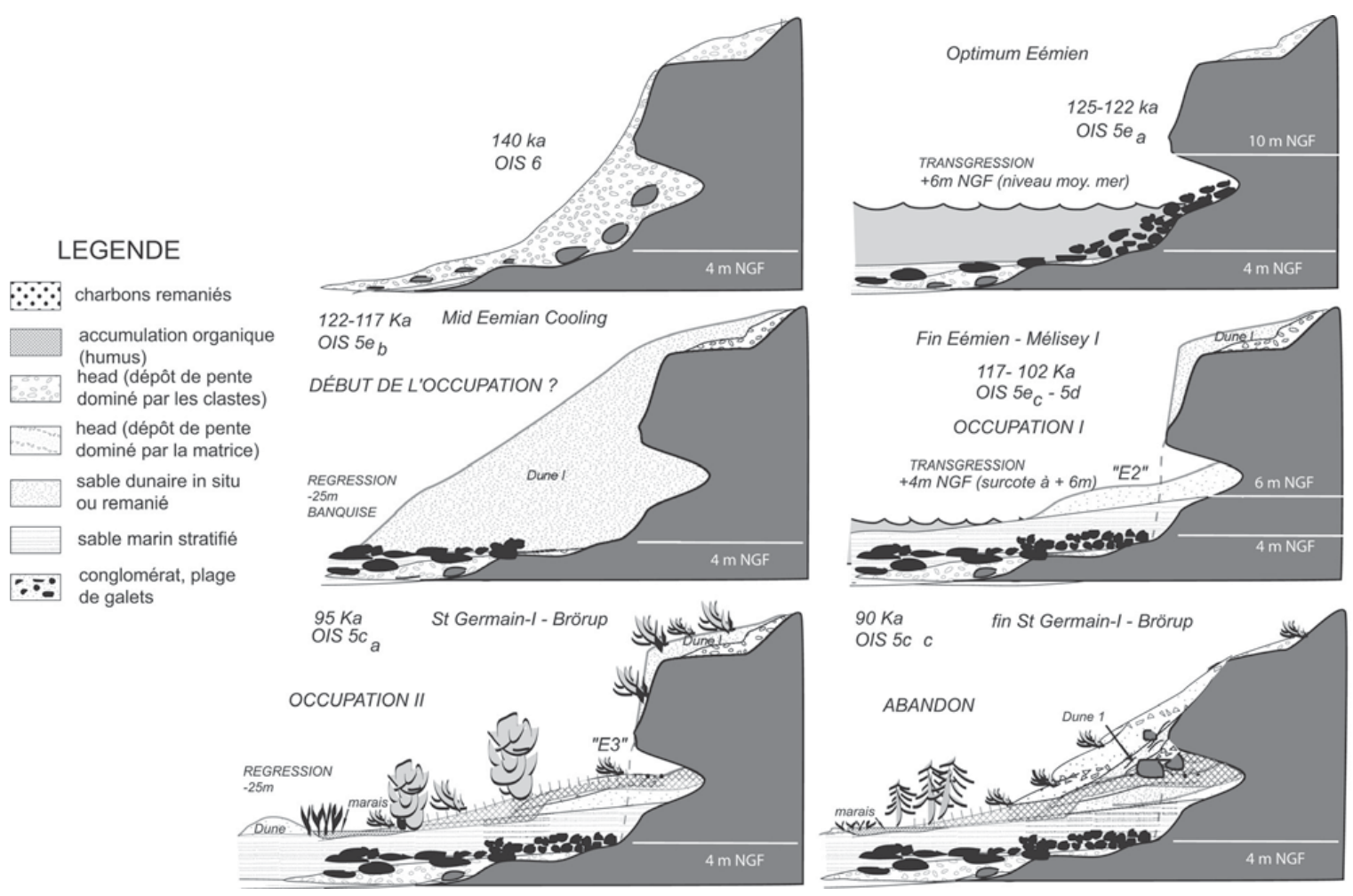

Fig. 29 : Reconstitution d'évolution environnementale du Rozel durant les stades isotopiques 6 à 5c (d'après B. Van Vliet-Lanoë). Fig. 29: Reconstitution of the environmental evolution of the Rozel site from OIS 6 to 5 c (from B. Van Vliet-Lanoë). 
l'Eemien et au début du OIS 5c, avant que la dégradation de la fin de cet interstade ne rende le site inhospitalier.

Par ailleurs, il n'existe qu'un évènement dunaire vers 115-113 ka, repris vers $107 \mathrm{ka}$. La mer n'est plus revenue ensuite. Par contre, ultérieurement, l'ensemble des matériaux provient essentiellement du remaniement ou de l'effondrement du massif dunaire fini éémien qui devait, comme aujourd'hui aux Mielles de Biville, se développer très haut sur le versant.

Les investigations conduites sur le site et sur les mobiliers lithiques et osseux permettent également de conclure en au moins deux périodes d'occupation. Notamment, le ré-examen de la faune atteste de grandes similitudes entre les deux séries fauniques, celle de l'abri et celle de la Grande Dune. Dans les deux ensembles, la faune est dominée par l'aurochs (Bos primigenius), puis le cerf élaphe (Cervus elaphus), et enfin le cheval (Equus sp.).

La première période d'occupation, associée à un pédocomplexe a livré une industrie caractéristique du paléolithique moyen (couches E2-D6-E3 de F. Scuvée) et des restes de faune consommée. Le mobilier lithique est confectionné dans du galet marin, issu des cordons littoraux; le débitage est orienté vers la production d'éclats, notamment prédéterminés, et d'enlèvements allongés. Les restes osseux conservés dans la Grande Dune apparaissent moins affectés par les agents physico-chimiques que ceux de l'abri. Les observations effectuées sur les cervidés plaident en faveur d'une occupation du site durant l'automne ou l'hiver. La récupération de bois de chute est aussi attestée dans la couche E2. A la faune consommée s'ajoute un fragment de symphyse mandibulaire de morse (Odobenus cf. rosmarus), pièce osseuse qui confère une certaine originalité au site du fait de la rareté des restes de pinnipèdes dans les niveaux anthropiques du Pléistocène supérieur. Selon B. Van Vliet-Lanoë, la préservation des ossements dans l'abri TR67 en raison du caractère carbonaté de la dune (115-113 ka) suggèrerait une occupation du site au moins à partir de la fin du Mid Eemian Cooling: les abris sont restés au-dessus du niveau atteint par les surcotes des tempêtes (D1c).

La seconde période d'occupation correspond au(x) sol(s) d'occupation démantelé(s) de l'abri TR67 qui conservai(en)t des éléments de structures de combustion (foyers et/ou vidanges de foyers). Le mobilier lithique apparaît constitué de quartz de filon, prélevé directement dans l'abri, et de galets marins collectés dans les cordons littoraux. Le système de production est orienté vers le débitage d'éclats (prédéterminés) et de lames (Levallois et de «type paléolithique supérieur »). L'utilisation de roches de substitution au silex s'avère très originale en Normandie armoricaine. Autre originalité de la série, la production d'éclats Levallois de très petites dimensions. Comme pour la plupart des assemblages lithiques du Cotentin, l'outillage apparaît peu abondant et la retouche discrète. L'assemblage osseux, mal conservé ne permet pas d'appréhender le cortège faunique dans sa globalité. Comme pour le complexe inférieur, la faune apparaît dominée par l'aurochs, les cervidés et le cheval, dans un environnement un peu plus steppique. Un frontal de cerf élaphe témoigne d'une occupation de l'abri à la fin de l'hiver ou au début du printemps.

Enfin, l'analyse de la faune nous renseigne sur les stratégies d'acquisition et de traitement des matières animales. Bien que statistiquement non représentatifs, les vestiges de faune consommée attestent que les activités de boucherie (fracturation des os longs, traces de découpe) se sont exercées aux dépens de l'aurochs et dans une moindre mesure du cerf, tant dans l'abri que dans la Grande Dune. Les portions de squelette plaideraient en faveur de modes d'acquisition différents ; les aurochs et les chevaux pourraient avoir été abattus à distance du site et rapportés par quartiers, alors que les cerfs paraissent avoir été rapportés entiers. Les stigmates inhérents aux activités de boucherie attestent d'une récupération maximum de nourriture (fracturations, traces de découpe) et réfutent l'existence de traces de carnivores sur les os conservés sur le site du Rozel.

Bien que la couche D6 de F. Scuvée n'ait livré aucun artefact lithique, la présence de restes de faunes affectés de traces anthropiques plaiderait en faveur d'une succession de trois ensembles témoignant d'une série d'occupations anthropiques durant une période tempérée du début du Dernier Glaciaire. L'étude de la faune confirme cette attribution chronologique, notamment les vestiges de chevaux. Le cortège faunique atteste d'un paysage en mosaïque de prairie-parc boisé, sous un climat nettement tempéré dominé par la «trilogie » aurochs-cerf-cheval, associé au Megaloceros au sein de l'abri et au morse dans les couches de la Grande Dune.

Comme nous l'avons évoqué précédemment, cette attribution chronologique s'avère confirmée par les observations géomorphologiques et fondée sur les datations radiométriques. Ces occupations sont datées de la première moitié du stade isotopique 5 par la technique de l'aliquot unique et les corrélations pédostratigraphiques régionales. L'analyse du mobilier lithique corrobore cette attribution chronologique. L'ensemble lithique collecté dans l'abri intègre le groupe des industries laminaires de «type paléolithique supérieur » du nord ouest de l'Europe. Ces dernières se développent durant le début du dernier glaciaire weichsélien.

Pour conclure, les occupations du Rozel s'avèrent exceptionnelles pour la Normandie, puisqu'elles conservent de la faune associée à des industries à composante mixte et à des structures d'occupation datées de la fin de l'Eemien et du début du Pléistocène supérieur. Elles pourraient correspondre soit à des sites de boucherie et /ou à des haltes de chasse, mais plus probablement à un habitat temporaire. 


\section{RÉFÉRENCES BIBLIOGRAPHIQUES}

ADKINS J.F., BOYLE E.A., KEIGWIN L., \& CORTIJO E., 1997 - Variability of the North Atlantic thermohaline circulation during the last interglacial period. Nature, 390, 154-158.

AUGUSTE P., 1995 - Cadres biostratigraphiques et paléoécologiques du peuplement humain dans la France septentrionale durant le Pléistocène. Apports de l'étude paléontologique des grands mammifères du gisement de Biache-Saint-Vaast (Pas-de-Calais). Thèse Doctorat Muséum National d'Histoire Naturelle de Paris, 5 tomes, $724 \mathrm{p}$.

AUGUSTE P., 2002 - Les vestiges fauniques. In J.-L. Locht (dir) «Bettencourt-Saint-Ouen (Somme). Cinq occupations paléolithiques au début de la dernière glaciation », Paris, Maison des Sciences de l'Homme, Documents d'Archéologie Française, 90, 39-47.

BALESCU S., \& LAMOTHE M., 1992 - The blue TL emission of $\mathrm{K}$-feldspar and its potential for overcoming TL age underestimation. Quaternary Science Reviews, 11, 45-51.

BALESCU S., PACKMAN S.C., \& WINTLE A.G., 1991 - Chronological separation of interglacial raised beaches from Northwestern Europe using thermoluminescence. Quaternary Research, $\mathbf{3 5}$ 91-102.

BATEMAN M.D., \& VAN HUISSTEDEN J., 1999 - The timing of the last-glacial periglacial and eolian events, Twente, eastern $\mathrm{Ne}-$ therlands. Journal of Quaternary Science, 14, 277-283.

BEAULIEU de J.L., \& REILLE M., 1992 - The last climatic cycle at La Grande Pile (Vosges, France). A new pollen profile. Quaternary Science Reviews, 11, 431-438.

BINFORD L.R., 1981 - Bones : ancient men and modern myths. New York, Academic Press, 320 p.

BINFORD L.R., 1988 - Etude taphonomique des restes fauniques de la Grotte Vaufrey, couche VIII. In RIGAUD J. (dir.) La Grotte Vaufrey, Mémoires de la Société Préhistorique Française, T. XIX, 535563.

BOSSCHA-ERDBRINK D.P., \& VAN BREE P.J.H., 1990 - Further observation on fossil and subfossil Odobenid material (Mammalia, Carnivora) from the North Sea. Beaufortia, 40, 85-101.

BRUGAL J.P., 1983 - Applications des analyses multidimensionnelles à l'étude du squelette des membres des grands Bovidés pléistocènes (Grottes de Lunel-Viel, Hérault). Perspectives évolutives. Thèse 3ème cycle Université Aix-Marseille II, 449 p.

CALLOW P., \& CORNFORD J.M., 1986 - La Cotte de Saint-Brelade (Jersey) 1961-1978. Excavations by C.B.M. Mc Burney. Norwich, Geobooks, 433 p.

CLET M., 1988 - Etude de séquences interglaciaires en Normandie corrélables aux stades isothopiques et 5 . Actes du Xe Symposium de l'Association des Palynologues de langue française, Bordeaux, 1987. Institut Français de Pondichéry éd., Travaux de la section scientifique et technique, XXV, 151-168.

CLIQUET D., \& LAUTRIDOU J.P., 2000 - Lœss et Paléolithique en Normandie (France du nord-ouest). Praehistoria, 1, 31-46.

CLIQUET D., \& MONNIER J.L., 1993 - Signification et évolution du Paléolithique moyen récent armoricain. Bulletin de la Société Préhistorique Française, 90, 275-282.

CLIQUET D., 1994 - Le Gisement paléolithique moyen de SaintGermain-des-Vaux / Port-Racine (Manche) dans son cadre régional. Essai palethnographique. Editions ERAUL, Liège, 63, 2 vol., $644 \mathrm{p}$.

CLIQUET D., MERCIER N., VALLADAS H., FROGET L. MICHEL D., VAN VLIET-LANOË B., \& VILGRAIN G., 2003 - Apport de la thermoluminescence sur silex chauffés à la chronologie de sites paléolithiques de Normandie : nouvelles données et interprétations. Quaternaire, 14, 1, 51-64.

CORTIJO E., DUPLESSY J.-C., LABEYRIE L., LECLAIRE H., DUPRAT J., \& VAN WEERING T.C.E., 1994 - Eemian cooling in the Norwegian Sea and North Atlantic Ocean preceeding continental ice-sheet growth. Nature, 372, 446-449.

COUTARD S., 2003 - Formations quaternaires en bordure d'une mer épicontinentale, la Manche. Tectonique, eustatisme, climat et occupations humaines. Exemple du Val de Saire (Normandie France). Université de Caen, U.F.R. Sciences. Thèse de Doctorat. $446 \mathrm{p}$
DE VERNAL A., EYNAUD F., HENRY M., HILLAIREMARCEL C., LONDEIX L., MANGIN S., MATTHIESSEN J., MARRET F., RADI T., ROCHON A., SOLIGNAC S., \& TURON J.L., 2004 - Reconstruction of sea-surface conditions at middle to high latitudes of the Northern Hemisphere during the Last Glacial Maximum (LGM) based on dinoflagellate cyst assemblages. Quaternary Science Reviews, 24, 897-924.

DIONNE J.C., 1998 - Sedimentary structures made by shore ice in muddy tidal-flat deposits, St Lawrence estuary, Québec. Sedimentary Geology, 116, 261-274.

DUGUY R., 1986 - Observation d'un morse (Odobenus rosmarus) sur la côte de Gironde, France. Mammalia, 50, 563-564.

EISENMANN V., 1991 - Les chevaux quaternaires européens (Mammalia, Perissodactyla). Taille, typologie, biostratigraphie et taxonomie. Geobios, 24, 747-759.

EMILIANI C., 1955 - Pleistocene temperatures. Journal of Geology, 63, 538-578.

FOLZ E., 2000 - La luminescence stimulée optiquement du quartz : développements méthodologiques et applications à la datation de séquences du Pléistocène supérieur du Nord-Ouest de la France. Thèse de doctorat, Université de Paris 7, 267 p.

FOSSE G., CLIQUET D., \& VILGRAIN G., 1986 - Le Moustérien du Nord-Cotentin (département de la Manche) : premiers résultats de trois fouilles en cours. Chronostratigraphie et faciès culturels du Paléolithique inférieur et moyen dans l'Europe du nord-ouest. Actes du Colloque International, Lille-Mons, 1984, sup. au Bulletin Association Française pour l'Etude du Quaternaire, 141-155.

FRONVAL T., \& JANSEN E., 1996 - Rapid changes in ocean circulation and heat flux in the Nordic seas during the last interglacial period. Nature, $\mathbf{3 8 3}, 806-810$.

GUADELLI J.L., 1991 - Les chevaux de Solutré (Saône et Loire, France). In «Datation et Caractérisation des Milieux Pléistocènes », Actes symposiums 11 et $1711^{\text {ème }}$ RST, Clermont-Ferrand, 1986, Cahiers du Quaternaire, 16, 261-336.

HALLÉGOUËT B., \& VAN VLIET-LANOË B., 1986 - Les Oscillations climatiques entre 125.000 ans et le maximum glaciaire, d'après l'étude des formations marines, dunaires et périglaciaires de la côte des Abers (Finistère). Bulletin Association Française pour l'Etude du Quaternaire, 25-26, 127-138.

HALLÉGOUËT B., \& VAN VLIET-LANOË B., 1989 - Héritages glaciels sur les côtes du Massif Armoricain, France. Géographie Physique et Quaternaire, 43, 223-232.

KEEN D.H., VAN VLIET-LANOË B., \& LAUTRIDOU J.P., 1996 - Two long sedimentary records from Jersey, Channel Islands : stratigraphic and pedological evidences for environmental change during the last $200 \mathrm{ky}$. Quaternaire, 7, 3-13.

KEEN D.H., 1980 - Raised beaches and shore plateforms in the Channel Islands. Brighton Polytech. Geogr. Soc. Mag ., 7, 10-15.

KERNEY M.P., \& CAMERON, R.A.D., 1979 - A field guide to the Land Snails of Britain and North-West Europe. London, Collins, $286 \mathrm{pp}$.

KOLSTRUP E., GRÜN R., MEJDAHL V., PACKMAN S.C., \& WINTLE AG., 1990 - Stratigraphy and thermoluminescence dating of Late Glacial cover in Denmark. Journal of Quaternary Science, 5, 207-224.

LABEYRIE J., 1984 - Le cadre paléoclimatique depuis 140.000 ans. L'Anthropologie, 88, 19-48.

LAUTRIDOU J.P., 1985 - Le Cycle périglaciaire pléistocène en Europe du nord-ouest et plus particulièrement en Normandie. Caen, Centre de géomorphologie, C.N.R.S., 2 vol., 907 p.

LAUTRIDOU J.P. et collaborateurs, 1986 - Les heads et les lœss du cycle récent en Cotentin. In Lœss et autres dépôts périglaciaires pléistocènes de l'Europe du Nord-Ouest. Relations avec les formations marines. Symposium de la Commission INQUA des loess et de la Commission UGI de la signification des phénomènes glaciaires, Normandie, Jersey, Bretagne, 1986, Centre de Géomorphologie du CNRS éd., 90-99.

LINSLEY B.K., 1996 - Oxygen isotope evidence of sea level and climatic variations in the Sulu Sea over the past 150,000 years. Nature, 380, 234-237. 
LOYER S., VAN VLIET-LANOË B., MONNIER J.L., HALLÉGOUËT B., \& MERCIER N., 1995 - La coupe de Nantois (baie de Saint-Brieuc, France) : datations par thermoluminescence et données paléo environnementales nouvelles pour le Pléistocène de Bretagne. Quaternaire, 6, 21-34.

MAC MANUS J.F., 1997 - Sultry Last Interglacial Gets a Sudden Chill. Earth in Space, Am Geophys. Union 9, 7,12-14.

MAC MANUS J.F., BOND G.C., BROECKER W.S., JOHNSEN S., LABEYRIE L., \& HIGGINS S., 1994 - High-resolution climate records from the North Atlantic during the last interglacial. Nature, 371, 326-329.

MANGERUD J., 2004 - Ice sheet limits on Norway and the Norwegian continental shelf. In : J.G. Ehlers \& P. Gibbard (Ed.), Quaternary glaciations extent and chronology, Elsevier, Amsterdam, 271-294.

MARTINSON D.G., PISIAS N.G., HAYS J.D., IMBRIE J., MOORE T.C., \& SHACKLETON N.J., 1987 - Age dating an the orbital theory of the Ice Ages : development of a high-resolution 0 to 333,000 year chronostratigraphy. Quaternary Research, 27, 1-29.

MASLIN M., 1996 - Intra-Eemian cold event. Terra Nova, 8, 5 .

MASLIN M., SARNTHEIN M., KNAACK J.J., GROOTES P., \& TZEDAKIS C., 1998 - Intra- interglacial cold events : an Eemian - Holocene comparison. In : A. Cramp, C.J. MacLeod, S.V. Lee \& E.J.W. Jones (Eds.), Geological Evolution of Ocean Basins : Results from the Ocean Drilling Program. Geological Society London, Special Publication, 131. 91-99.

MEURISSE M., \& VAN VLIET-LANOË B., 2004 - Evolution du littoral picard durant l'Holocène. Sucellus, 55, 17-31.

MEURISSE M., VAN VLIET-LANÖ̈ B., TALON B., \& RECOURT P., 2005 - Complexes dunaires et tourbeux holocènes du littoral du Nord de la France. CR Géosciences, 337, 675-684.

MERCIER N., VALLADAS H., \& FROGET L., 2001 - La méthode de datation par luminescence stimulée optiquement (OSL) : développements récents et perspectives d'applications. In : J-N. Barrandon, P. Guibert \& V. Michel (dir.), Datation. XXIe Rencontres Internationales d'Archéologie et d'Histoire d'Antibes, Editions APDCA - Antibes, 49-55.

MERCIER N., VALLADAS H., \& VALLADAS G., 1992 - Some observations on palaeodose determination in burnt flints. Ancient $T L, \mathbf{1 0}, 28-32$.

MONNIER J.L., \& VAN VLIET-LANOË B., 1986 - Les oscillations climatiques entre 125000 ans et le maximum glaciaire d'après l'étude des coupes du littoral de la Baie de Saint Brieuc. Apport de la lithologie, de la pédologie et de la malacologie. Bulle tin de l'Association Française pour l'Étude du Quaternaire, 25 26, 119-126.

MONNIER J.L., 1980 - Le Paléolithique de la Bretagne dans son cadre géologique. Rennes, Travaux du Laboratoire d'Anthropologie, Préhistoire, Protohistoire et Quaternaire Armoricain, C.N.R.S., 607 p.

MONNIER J.L., 1985 - Données nouvelles sur le gisement paléolithique moyen de Piégu (Pléneuf-Val-André, Côtes-du-Nord). Revue archéologique de l'Ouest, 2, 7-21.

MONNIER J.L., FALGUÈRES C., LAURENT M., BAHAIN J.J., MORZADEC-KERFOURN M.T., \& SIMONET P., 1995 - Analyses des données anciennes et contributions nouvelles à la connaissance et à la datation du gisement moustérien de Mont-Dol (Ille-et-Villaine). In L. Langouët \& M.-T. Morzadec-Kerfourn (dir.), Baie du Mont-Saint-Michel et Marais de Dol, milieux naturels et peuplements dans le passé, Centre régional d'archéologie d'Alet, Saint-Malo, 3-26.

MUHS D.R., SIMMONS K.R., KENNEDY G.L., \& ROCKWELL T.K., 2001 - The last interglacial period on the Pacific Coast of North America : Timing and paleoclimate. Geological Society of America Bulletin, 114, 569-592.

OPPO D.W., HOROWITZ M., \& LEHMAN S.J., 1997 - Marine evidence for reduced deep water production during Termination II followed by a relatively stable substage $5 \mathrm{e}$ (Eemian). Paleoceanography, 12, 51-63.

OVERPECK, J.T., OTTO-BLIESNER, B.L., MILLER, G.H., MUHS, D.R., ALLEY, R.B., \& KIEHL, J.T., 2006 - Paleoclimatic Evidence for Future Ice-Sheet Instability and Rapid Sea-Level Rise. Science, 311, 1747-1750.
PEDERSEN A., 1974 - Famille des Odobénidés (Morses). In : B. Grzimek (ed.), Le Monde animal en 13 volumes, Stauffacher, Zurich, 12, 346-351.

PROCTOR C.J., \& SMART P.L., 1991 - A dated cave sediment record of Pleistocene transgressions on Berry Head, Southwest England. Journal of Quaternary Science, 6, 233-244.

REVILLION S., \& TUFFREAU A. (Dir.), 1994 - Les industries laminaires au Paléolithique moyen. Actes de la Table-ronde de Villeneuve d'Ascq, 13 et 14 novembre 1991, Paris C.N.R.S. éd., Dossier de documentation archéologique du CRA, $192 \mathrm{p}$.

ROUSSEAU D.D., \& KEEN D.H., 1989 - Malacological records from the Upper Pleistocene at Portelet (Jersey, Channel Islands) comparisons with western and central Europe. Boreas, 18, 61-66.

SANCHEZ-GOÑI M.F., EYNAUD F., TURON J.L., \& SHACKLETON N.J., 1999 - High resolution palynological record of the Iberian Margin : direct land-sea correlation for the last interglacial complex. Earth \& Planetary Science Letters, 171, 123-137.

SCOURSE J.D., 1996 - Late Pleistocene stratigraphy of North and West Cornwall. Philosophical Transactions of the Royal Society of Cornwall, 22, 1-56.

SCUVEE F., 1967 - Le Rozel : Trou du renard. Rapport de fouilles ronéoté, $10 \mathrm{p}$.

SCUVEE F., 1969 - Le Rozel : Trou du renard. Rapport de fouilles ronéoté, $12 \mathrm{p}$.

SCUVEE F., \& VERAGUE J., 1984 - Paléolithique supérieur en Normandie occidentale : l'abri sous-roche de la pointe du Rozel (Manche). Cherbourg, LITTUS-C.E.H.P., 150p.

SCUVEE F., \& VERAGUE J., 1988 - Note complémentaire relative à l'abri sous roche de la pointe du Rozel (Manche). Cherbourg, LITTUS-C.E.H.P., 36-37, 41-44.

SHACKLETON N.J., 1986 - The Plio-Pleistocene ocean : stable isotope history. In : Mesozoïc and Cenozoüc oceans, Geodynamics series, 15, 141-153.

SHACKLETON N.J., SÁNCHEZ-GOÑI M.F., PAILLER D., \& LANCELOT Y., 2003 - Marine Isotope Substage 5e and the Eemian Interglacial. Global and Planetary Change, 36, 151-155.

SONNEVILLE-BORDES D. de, \& LAURENT P., 1983 - Le phoque à la fin des temps glaciaires. In POPLIN F. (dir.), La faune et l'Homme préhistoriques, Mémoires de la Société Préhistorique Française, 16, 69-80.

VALLADAS H., 1992 - Thermoluminescence dating of flint. Quaternary Science Reviews, 11, 1-5.

VAN VLIET-LANOË B., 1986 - Le pédocomplexe du Dernier Interglaciaire (de 125000 à $75000 \mathrm{BP}$ ). Variations de faciès et signification paléoclimatique, du sud de la Pologne à l'ouest de la Bretagne. Bulletin Association Française pour l'Etude du Quaternaire, 1-2, 139-150.

VAN VLIET-LANOË B., 1988 - Le rôle de la glace de ségrégation dans les formations superficielles de l'Europe de l'Ouest. Processus et héritages. Editec, Caen, 1988, 854 p.

VAN VLIET-LANOË B., \& GUILLOCHEAU F., 1995 - Evolution de l'enregistrement pédo-sédimentaire depuis 150 ka en France du nord-ouest et en Belgique : biorhexistasies et bilans sédimentaires. Comptes rendus de l'Académie des sciences (IIa), 320, 419426.

VAN VLIET-LANOË B., LAURENT M., BAHAIN J.J., BALESCU S., FALGUÈRES C., FIELD M., HALLÉGOUËT B., \& KEEN D.H., 2000 - Middle Pleistocene raised beach anomalies in the english Channel : regional and global stratigraphy implications. Journal of Geodynamic, 29, 15-41.

VAN VLIET-LANOË B., TUFFREAU A., \& CLIQUET D. 1993 Position stratigraphique des industries moustériennes à lames en Europe Occidentale. In: A. Tuffreau (dir.), Riencourt-lès-Bapaume (Pas-de-Calais). Un gisement Paléolithique moyen. Documents d'Archéologie Française, 37, 104-106.

VAN VLIET-LANOË B., VANDENBERGHE N., LAIGNEL B., LAURENT M., LAURIAT-RAGE A., LOUWYE S., MANSY J.L., MEILLIEZ F., MERCIER D, HALLEGGUËT B., LAQUEMENT F., MICHEL Y., \& MOGUEDET G, 2002 - Paleogeographic evolution of the Western Europe during the Upper Cenozoic. Geodiversitas 24, 511-541. 